\title{
Microarcsecond Astrometry: Science Highlights from Gaia
}

\section{Anthony G.A. Brown}

Leiden Observatory, Leiden University, Niels Bohrweg 2, 2333 CA Leiden, The Netherlands; email: brown@strw.leidenuniv.nl

Xxxx. Xxx. Xxx. Xxx. YYYY. AA:1-61

https://doi.org/10.1146/((please add article doi))

Copyright (C) YYYY by Annual Reviews. All rights reserved

\section{Keywords}

astrometry, space vehicles: Gaia, catalogs, surveys

\section{Abstract}

Access to microarcsecond astrometry is now routine in the radio, infrared, and optical domains. In particular the publication of the second data release from the Gaia mission made it possible for every astronomer to work with easily accessible, high-precision astrometry for 1.7 billion sources to 21 st magnitude over the full sky.

- Gaia provides splendid astrometry but at the limits of the data small systematic errors are present. A good understanding of the Hipparcos/Gaia astrometry concept, and of the data collection and processing, provides insights into the origins of the systematic errors and how to mitigate their effects.

- A selected set of results from Gaia highlight the breadth of exciting science and unexpected results, from the solar system to the distant universe, to creative uses of the data.

- Gaia DR2 provides for the first time a dense sampling of Galactic phase space with high precision astrometry, photometry, and radial velocities, allowing to uncover subtle features in phase space and the observational HR diagram.

- In the coming decade, we can look forward to more accurate and richer Gaia data releases, and new photometric and spectroscopic surveys coming online that will provide essential complementary data.

- The longer term promises exciting new opportunities for microarcsecond astrometry and beyond, including the plans for an infrared version of Gaia which would offer the dense sampling of phase space deep into the Milky Way's nuclear regions. 


\section{Contents}

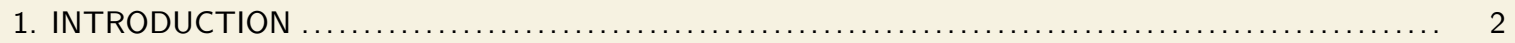

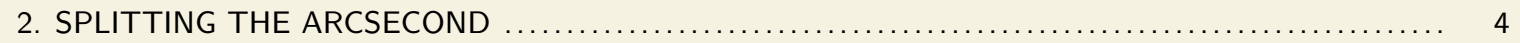

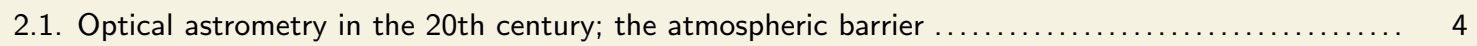

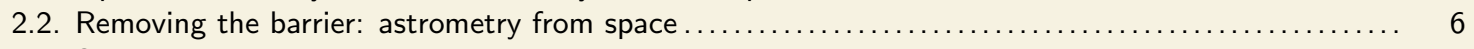

2.3. Splitting the milliarcsecond with interferometry $\ldots \ldots \ldots \ldots \ldots \ldots \ldots \ldots \ldots \ldots \ldots \ldots \ldots \ldots \ldots \ldots, 7$

3. GLOBAL ASTROMETRY WITH THE HIPPARCOS/GAIA CONCEPT $\ldots \ldots \ldots \ldots \ldots \ldots \ldots \ldots \ldots \ldots . \ldots$

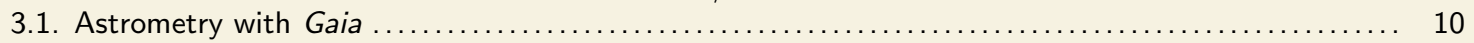

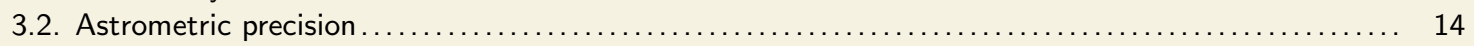

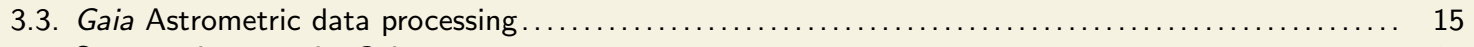

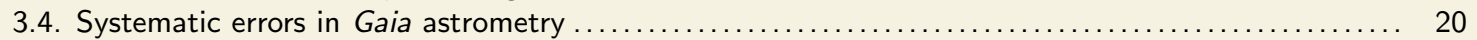

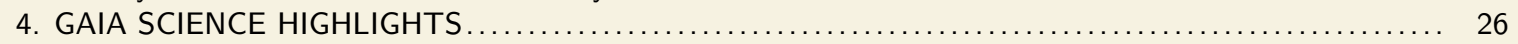

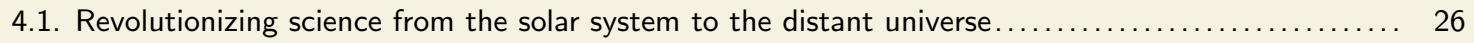

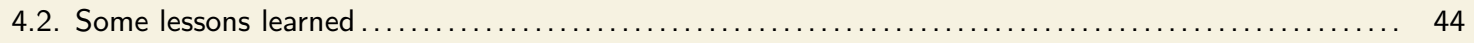

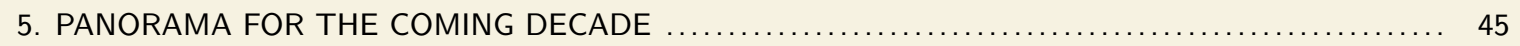

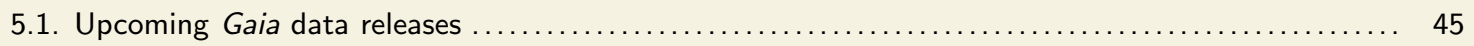

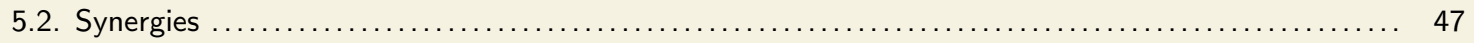

6. FUTURE DIRECTIONS FOR MICROARCSECOND ASTROMETRY $\ldots \ldots \ldots \ldots \ldots \ldots \ldots \ldots \ldots \ldots \ldots . . \ldots$

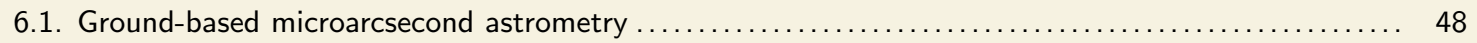

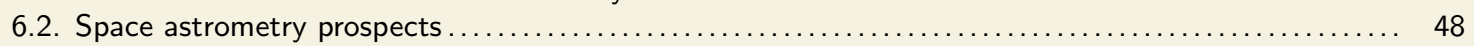

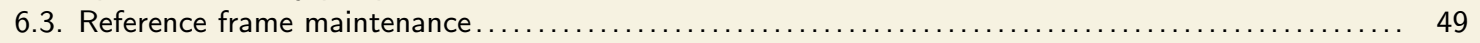

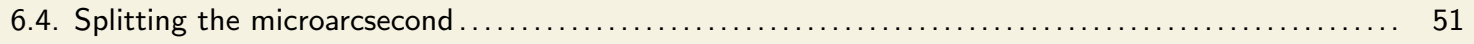

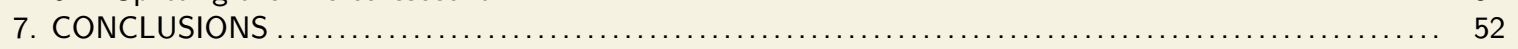

\section{INTRODUCTION}

Advances in astrometric techniques and instrumentation over the past two decades have brought us to the point where the measurement uncertainties have become so small as to make it convenient to express these in units of microarcseconds (where $1 \mu$ as corresponds to 5 picoradians). In this review "microarcsecond astrometry" refers to instruments and surveys that routinely reach astrometric measurement uncertainties at the tens of $\mu$ as level. These include the radio VLBI technique, the GRAVITY instrument in the infrared, and the Hubble Space Telescope in the optical in its spatial scanning mode. However, above all it is the Gaia mission and its second data release which truly opened up the microarcsecond era in the optical domain, revolutionizing all fields of astronomy.

This review has two main objectives, to explain "how Gaia works" and to summarize a selection of science highlights from the first two Gaia data releases. The aim of reviewing the Hipparcos/Gaia concept of making absolute astrometric measurements is to provide the astronomer using the Gaia data with a basic understanding of how the data is collected and processed. The main drivers of the precision of the measurements are explained and particular attention is paid to the sources of systematic errors in the Gaia astrometry and how these can be mitigated. The resulting enhanced understanding of the Gaia catalogue data will improve the scientific interpretation thereof.

Microarcsecond astrometry opens up an enormous number of exciting science areas. At radio wavelengths, the VLBI technique allows for establishing geometric distances to distant star forming regions which can be used to trace the Milky Way's spiral arms. In the

2 Anthony G.A. Brown 
infrared domain, exquisite studies of the dynamics of stars orbiting the Milky Way's central black hole are possible with the GRAVITY instrument, leading to an incredibly precise determination of the distance to the Galactic centre as well as tests of general relativity. At optical wavelengths, microarcsecond astrometry enables a recalibration of the distance scale of the universe through geometric distances to standard candles (HST, Gaia), and the Gaia data alone provide a fantastic showcase of the power of the highly accurate fundamental astronomical data, positions, parallaxes, and proper motions. The first and second Gaia data releases, Gaia DR1 and Gaia DR2, have revolutionized the studies of the structure, dynamics, and formation history of the Milky Way (see for example the review by Helmi 2020), but have made possible much more beyond this core goal of the Gaia mission.

Precise star positions enable the study of the shapes and possibly atmospheres of Kuiper Belt Objects through stellar occultations. The accurate Gaia parallaxes for large numbers of stars reveal subtle and as yet unexplained features in the observational Hertzsprung-Russell diagram, and allows us to peer deep into the interiors of white dwarfs. The dense sampling with parallaxes and proper motions (and Gaia radial velocities) of the phase space around the sun uncovered the "phase spiral" and the exciting story of the interaction between the Sagittarius dwarf galaxy and the Milky Way. The Gaia proper motion measurements in distant stellar systems allow the detailed mapping of globular cluster and satellite galaxy orbits, the uncovering of the many new streams, and provides us with the equivalent of an integral field unit measurement of the tangential motion fields in the large and small Magellanic clouds. Finally, Gaia reaches all the way to distant universe, providing discoveries of new lensed quasar systems and insights into AGN accretion disk and jets. The other main objective of this review is thus to summarize these and other science highlights from Gaia. Only a highly selected number of science topics will be discussed, intended primarily to illustrate the breath of science that can be addressed with Gaia data, hopefully inspiring further creative uses of the data. Many important topics are missing, which is entirely the choice of the author, and the absence of a particular topic does not in any way reflect on its relevance.

This review starts in Section 2 with a brief historical overview of 20th century astrometry, focused on motivating the need for astrometry from space. The modern context for the Gaia mission is also provided, discussing several instruments and techniques that are highly complementary to Gaia and can outperform it in astrometric precision. Section 3 explains in some detail how the Hipparcos/Gaia concept of astrometry works. Section 3.1 explains the measurement concept, which is followed in Section 3.2 by a discussion of the drivers of the astrometric precision of Gaia and how these can be used to make mission parameter trade-offs. Section 3.3 discusses the astrometric data processing for Gaia and is focused on providing the elements needed to understand the origin of systematic errors in Gaia astrometry, which are discussed in Section 3.4. The main aim of Section 3 is to provide the user of the Gaia data with enough understanding of the mission and its measurement concepts to profit from this knowledge when interpreting the Gaia data. For the interested reader many entry points to the Hipparcos/ Gaia literature are provided in which much more details can be found. Section 4 presents selected science highlights, mostly from Gaia DR2, and the topics are roughly ordered by "distance", from the solar system to the quasars. In addition some of the more unexpected uses of the Gaia data are highlighted. Section 4 closes with a few lessons learned so far from the scientific exploitation of the Gaia data. Section 5 presents the prospects for the coming decade in which many more results from Gaia will appear, increasing in accuracy and richness, alongside the large spectroscopic 
Astrometry: The branch of astronomy concerned with the accurate measurement of celestial positions of astronomical sources.

Parallax: Apparent annual motion of a source on the sky as an observer orbits around the solar system barycentre.

Proper motion: Displacement of a source on the sky due to its motion with respect to the solar system barycentre.

Celestial reference system: Set of prescriptions and conventions together with the modelling required to define, at any time, a triad of axes.

Celestial reference frame: Practical realization of a celestial reference system defined by fiducial directions in agreement with the reference system's concepts. and photometric surveys soon starting their operations. In Section 6 future directions for astrometry are examined, including the essential task of maintaining the dense and highly accurate optical astrometric reference frame provided by Gaia. Section 7 summarizes the conclusions and highlights a number of important issues to address in the future.

\section{SPLITTING THE ARCSECOND}

Optical astrometric programmes from the 20th century are summarized, emphasizing the motivations for space astrometry ${ }^{1}$ The focus is on the state of affairs prior to the appearance of the Hipparcos Catalogue in 1997. Subsequent developments in ground based optical astrometry are not discussed. An extensive overview of the historical developments is provided by Perryman (2012). Modern radio and optical/IR interferometric instruments, which can outperform Gaia in terms of astrometric precision, are briefly described, stressing the powerful combination of different microarcsecond astrometry techniques.

\subsection{Optical astrometry in the 20th century; the atmospheric barrier}

Prior to the Hipparcos mission (ESA 1997), the discipline of astrometry was divided into three fields (Perryman 2012): parallax programmes to establish the distances to stars; large-scale surveys to collect positions and proper motions in support of Galactic structure studies; and the construction of astrometric reference frames through the measurement of precise absolute positions of a limited number of stars spread over the whole sky.

The highest relative astrometric measurement accuracies were needed for parallax programmes. These relied on measuring the parallactic motion of a target star through differential position measurements with respect to a presumably very distant reference star. The reviews by Vasilevskis (1966), van de Kamp (1971), and van Altena (1983) illustrate the major efforts that went into improving the long-focus astrometry technique, for which the principles were established at the start of the 20th century (Perryman 2012). Prior to the publication of the Hipparcos Catalogue, the state of the art was "The general catalogue of trigonometric parallaxes" (van Altena et al. 1995), which listed parallaxes for just over 8000 stars. The quoted uncertainties were smaller than 2 milliarcseconds for 10 percent of the sample (the mode of the uncertainty distribution was located at $\sim 10$ mas). Apart from the large uncertainties (for present day standards), the catalogue also suffered from the inhomogeneity of the observations and data underlying the parallax results.

All-sky surveys took off at the end of the 19th century. This was driven by the advent of photography that enabled the positions of many stars to be measured simultaneously, at the expense of abandoning the highest positional accuracies that were achievable with parallax programmes. Repeating such position measurements over time enabled the derivation of proper motions. The plate material from these surveys was eventually digitized, which allowed the construction of astrometric catalogues listing positions for up to hundreds of millions of sources and proper motions for tens of millions. Examples include the series of catalogues from the US Naval Observatory and the Guide Star Catalogue produced to

\footnotetext{
${ }^{1}$ The section title refers to the reply by C. Wynne, when asked about the design of the Hipparcos telescope, "I'm not saying that it won't [work] but I do know that seconds of arc don't split into milliseconds of arc very easily!" (quoted in Perryman 2010). This was paraphrased by L. Lindegren at the IAU Symposium 248 A Giant Step: From Milli- to Micro-Arcsecond Astrometry to remind the participants that "Milliarcseconds do not split into microarcseconds very easily".
}

$4 \quad$ Anthony G.A. Brown 


\section{Relative vs. absolute astrometry}

The terms "relative" and "absolute" astrometry are regularly contrasted. The former is often also referred to as "narrow-angle" astrometry and the latter as "global" or "wide-angle" astrometry. In relative astrometry the position of the target source is determined relative to a nearby (within less than $\sim 1^{\circ}$ ) reference source. This allows for the elimination of many sources of error and offers the highest astrometric precision. For applications where the absolute position of the sources is not relevant, such as exoplanet searches, this is the method of choice. It is also employed in all ground-based parallax programmes. The major drawback is that the reference source may have a non-zero parallax, which necessitates estimating corrections to the parallax of the target in order to put it on an absolute scale. This involves the uncertain modelling of the distribution of parallaxes of the reference source population and thus leads to lower accuracy. Choosing a distant extragalactic reference tends to severely restrict the sky area accessible to parallax measurements.

Absolute astrometry involves the measurement of directions to sources widely separated on the sky in order to establish their positions with respect to a fixed reference frame, instead of each other. The term "absolute" indicates that the positions and proper motions are given with respect to a quasi-inertial coordinate system with a known reference plane and pole (the $x-y$ plane and the direction of the $z$-axis, loosely speaking). A quasi-inertial system should not rotate. This is essential for the dynamical interpretation of the proper motions which assumes the absence of any centrifugal or Coriolis forces that would appear in a rotating frame. Absolute astrometry requires a known and stable reference platform from which the observations are carried out. In practice one solves for the source positions and the parameters of the observing platform at the same time (in a "global" solution).

In the case of Hipparcos and Gaia the use of two telescopes with viewing directions separated by a large angle $\left(\sim 90^{\circ}\right)$ establishes a network of angles measured between sources widely separated on the sky. Parallactic motions of different sources can then be disentangled which allows the measurement of absolute parallaxes, without reference to distant extragalactic sources (Section 3.1.1).

support Hubble Space Telescope operations. For an extensive overview of astrometric sky surveys from the 20th century refer to Perryman (2009).

The third astrometric task concerns the construction of an all-sky network of stars for which accurate absolute positions and proper motions are known. Such networks can be used to construct reference frames which serve as anchors for all other astrometric measurements. Throughout the 20th century the instrument of choice for reference frame observations remained the meridian circle, which collects position measurements of stars by precisely timing their transit across an observatory's local meridian. The consequence was that only a sparse network of reference stars could be established at relatively bright magnitudes. The enormous efforts to construct the optical astrometric reference frame prior to Hipparcos culminated with the fifth edition of the "Fundamental Katalog" (FK5 Fricke et al. 1988), which listed the positions and proper motions for 1535 stars, with the majority at $V$ magnitudes between 4 and 11 .

Astrometric surveys and reference frame programmes required collecting observations with different telescopes spread over different sites around the globe. Efforts were made to use the same telescope/instrument designs or the same measurement technique. Nevertheless astrometric catalogues based on inhomogeneous observations (from different telescope/instrument combinations at different observing sites) inevitably led to severe sys- 
tematic limitations to the accuracies of the surveys and the reference frame. These were manifest in the so-called "zonal" or "regional" errors which led to, among others, systematic proper motion biases which differ from one region on the sky to another. Local systematic errors in the positions in the Guide Star Catalogue version 1.1 (referenced to the FK5) were of the order of 1 arcsecond (Perryman 2009), while the distortions in the FK5 reference frame reached 100 mas or more (e.g. Schwan 2002). In addition Lindegren (1980) showed that traditional (long-focus) methods of narrow-angle differential astrometry were also limited by the effects of the Earth's atmosphere, predicting that at best parallaxes with precisions of order 1 mas could be collected at the rate of 100 per year.

Thus optical astrometry was in danger of stalling at positional accuracies at the arcsecond level for wide field surveys, corresponding to proper motion accuracies of at best

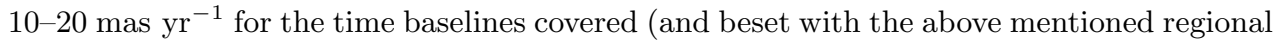
errors), and parallaxes limited to milliarcsecond level precision for modest numbers of stars (suffering from the accuracy limitations inherent to relative parallax measurements). The best existing reference frame was sparse and covered only stars of fairly bright magnitudes. The need for denser and fainter optical reference frames was stressed by Monet (1988), in particular to enable accurate pointing of the Hubble Space Telescope to ensure its high imaging resolution could be used together with other high angular resolution instruments, such as the Very Large Array. This remains an important issue as discussed in Section 6.3 .

\subsection{Removing the barrier: astrometry from space}

The unsatisfactory state of affairs for optical astrometry was recognized already in the 1960s when ideas started to be developed for overcoming the limitation of the Earth as an observing platform, by going to space (Perryman 2011). In 1967 P. Lacroute presented the proposal for what eventually became the Hipparcos mission (Perek 1967). The proposed concept solved several problems in one go (Perryman 2012):

- The move to space would ensure that the measurements were not hampered by the effects of the Earth's atmosphere and that the instruments would work in a thermally stable, gravity-free environment, thus eliminating two major causes of systematic errors in ground based astrometric surveys.

- A single instrument could be used to observe the entire sky, ensuring the homogeneity of the survey and further eliminating causes for zonal errors.

- The use of two telescopes with viewing directions separated by a wide angle of order 90 degrees, and with the images projected onto a common focal plane, enabled the construction of a rigid reference frame spanning the entire sky and the measurement of absolute as opposed to relative parallaxes.

It is this concept that provided both the Hipparcos and Gaia missions with the following key capabilities, rolling the three primary tasks of astrometry into one survey:

- The efficient collection of high accuracy absolute parallaxes for large numbers of stars over a wide range of magnitudes.

- A homogeneous and highly accurate survey of positions and proper motions for the same stars, free from zonal errors.

- The establishment of a dense, accurate, and rigid network of reference positions on the celestial sphere, free from regional errors. In the case of Gaia for the first time the optical celestial reference frame is realized directly through observations of distant 


\section{Astrometry with the Hubble Space Telescope}

Differential astrometry from space has been carried out with the Hubble Space Telescope since the 1990s. Its fine guidance sensors have been used to measure parallaxes to sub-milliarcsecond uncertainty levels (Benedict et al. 2017), while the cameras have been used to measure proper motions through imaging campaigns spread over several years. These programmes enabled studies of the internal kinematics of globular clusters (e.g. Bellini et al. 2015) and the Magellanic Clouds (van der Marel \& Kallivayalil 2014), and the tangential motions of dwarf galaxies (e.g. Kallivayalil et al. 2006) and M31 (Sohn et al. 2012). The publication of Gaia DR1 allowed for the anchoring of the HST astrometry to a dense and much more accurate net of reference sources, facilitating the measurement of absolute proper motions and, for example, the 3D internal motions in the Sculptor dwarf galaxy (Massari et al. 2018). Based on a new astrometric technique that employs spatial scanning with the HST (Riess et al. 2014, Casertano et al. 2016), Riess et al. (2018) presented parallax measurements of Cepheid variables at 30-50 $\mu$ as precision. HST thus remains very complementary to Gaia, especially for bright star parallax work and for proper motion studies of the most crowded regions in globular clusters, the Milky Way bulge, and the Magellanic Clouds.

$$
\text { QSOs (Gaia Collaboration et al.2018e). }
$$

A key element of Hipparcos and Gaia astrometry is the precise determination of source image locations in the data stream. This process also produces image fluxes, but accurate determination of either image parameter requires the knowledge of source colours in order to account for instrument chromatic effects. In practice a precise, simultaneous, and homogeneous multi-colour photometric survey is required to complement the astrometry. Thus Hipparcos and Gaia provide astrophysical information for all observed sources, in the case of Gaia also through the medium resolution spectra gathered by its Radial Velocity Spectrograph (Gaia Collaboration et al. 2016b, Cropper et al. 2018).

\subsection{Splitting the milliarcsecond with interferometry}

The limitations to ground-based optical astrometry can also be overcome through interferometry, which first came to fruition in the radio domain (Counselman 1976). Today, $\mu$ as astrometric precision is possible from Earth with radio or infrared interferometry. This technique combines the electromagnetic signal received by telescopes separated by a long baseline in order to achieve the necessary sensitivity to the exact direction to a source. Reid \& Honma (2014) reviewed microarcsecond astrometry with Very Long Baseline Interferometry, while Johnston \& de Vegt (1999) discussed the application of VLBI to the construction of reference frames. In the infrared domain the GRAVITY instrument coupled to ESO's Very Large Telescope Interferometer is capable of achieving microarcsecond astrometry (Gravity Collaboration et al. 2017).

2.3.1. Narrow angle astrometry. With VLBI single measurement positional precisions down to $\sim 10 \mu$ as can be achieved through relative measurements over narrow angles $\left(\sim 1^{\circ}\right)$ on the sky. Reid \& Honma (2014) outline the principles of the technique. One observes the target and reference source located close together on the sky at nearly the same time. For both sources the delay between the arrival time of the signal at one of the telescopes 
is measured. Differencing the two measurements allows one to eliminate sources of error due to delays from the troposphere, ionosphere, the antenna location uncertainties, and instrumental delays, as all these terms are very similar over small angles on the sky. Errors due to source structure can be handled by examining the source images and calculating the expected phase shifts. The errors due to thermal noise can be ignored for sufficiently high signal to noise ratio measurements.

The GRAVITY instrument combines the light from ESO's four VLT unit telescopes and can also employ the auxiliary telescopes on the Paranal site. A technique similar to VLBI is used to achieve high precision narrow angle astrometry, in this case over fields of view of 2-4 arcseconds. This again allows for the cancellation of several sources of error by differencing the target and reference source measurements. The operation at infrared wavelengths demands a much more complex instrument to control the phase differences (delays) between target and reference. GRAVITY features a variety of innovations which allow reaching microarcsecond astrometry (Gravity Collaboration et al. 2017). For broad band observations the GRAVITY instrument has been demonstrated to achieve single measurement positional precisions in the 30-100 $\mu$ as range (Gravity Collaboration et al. 2019). Over a sufficiently narrow wavelength range, the spectro-differential astrometry technique allows reaching relative positional precisions of a few $\mu$ as by tracking the phase differences between continuum and line emission from a source (Gravity Collaboration et al. 2017).

The interferometric instruments achieve astrometric precisions over narrow angles that surpass the performance of Gaia, and with a suitably distant reference source (such as QSOs) the relative astrometry can be placed on an absolute scale. Gaia and the interferometry instruments are highly complementary. Gaia provides access to astrometry for vast numbers of sources over the entire sky, but its astrometry is limited, or not available, in obscured regions in or near the Galactic plane, and in crowded areas such as the centres of globular clusters. Reid \& Honma (2014) summarize the science applications of VLBI astrometry which complement Gaia, such as: the accurate determination of distances and motions of maser sources in star forming regions, which allow us to trace the spiral arms of the Milky Way; the access to astrometry for asymptotic giant branch giant stars through masers in their envelopes (where Gaia astrometry is affected by the photocentre displacements due to the large convective atmospheres, Chiavassa et al. 2018); the access to astrometry of pulsars; and the possibility to study megamasers in other galaxies to make direct estimates of the Hubble constant. Astrometry from GRAVITY has been used in combination with older adaptive optics data, radial velocities, and the VLBI proper motion measurements of Sgr A*, to very accurately model the orbit of the S2 star around the massive black hole and derive a distance to the Galactic centre with only $0.3 \%$ uncertainty (Gravity Collaboration et al. 2019). This fixes a Galactic structural parameter which can be used in Milky Way studies with Gaia data. These synergies highlight the powerful combination of microarcsecond astrometry techniques at the disposal of astronomers in the 21st century.

2.3.2. Reference frames. On January 11998 the International Astronomical Union adopted the International Celestial Reference System (ICRS) as a celestial reference system based on directions to a set of extragalactic sources. The ICRS represents a quasi-inertial reference system to replace the older systems (e.g., FK5) in which source coordinates were referred to 
a system primarily based on the dynamics of the solar system (Feissel \& Mignard 1998 ${ }^{2}$ The practical materialisation of the ICRS is the Internal Celestial Reference Frame (ICRF), which consists of a list of celestial positions and their uncertainties for a set of extragalactic sources. For details on reference systems refer to Johnston \& de Vegt (1999).

The ICRF was first set up in the radio domain through VLBI astrometry of carefully selected extragalactic radio sources (Ma et al. 1998), observed over decades. Although the basic observables are the same as for narrow angle VLBI astrometry (delays in the signal as received by different antennae), a global solution must be made where the source positions are solved for along with the positions and velocities of the observing stations, accounting for the Earth's deformations and its orientation at the time of observation. In addition the effects of the ionosphere and troposphere are calibrated or modelled out (where the troposphere is one of the main factors limiting the astrometric accuracy). For details refer to Ma et al. (1998) and the chapter by Fomalont in van Altena (2013). The most recent version of the reference frame is the ICRF $3^{3}$ which consists of 303 "defining" sources and an additional 4285 sources which are being observed regularly and may enter the defining set at some future time. The median position uncertainty for the ICRF3 (as given by the semi-major axis of the uncertainty ellipse) is 0.23 mas for the full set of sources and $50 \mu$ as for the defining sources observed in the $8.4 \mathrm{GHz}$ band ( $80 \mu$ as for ICRF2). Hence also reference frames have now firmly entered the microarcsecond era.

Upon the introduction of the ICRF the IAU adopted the Hipparcos Catalogue as the optical materialisation of the ICRS (Feissel \& Mignard 1998). The optical frame was aligned to the radio frame (considered as the primary reference) through several intermediate steps, given that no extragalactic sources (except 3C 273) were observed by Hipparcos (Lindegren \& Kovalevsky 1995). Gaia observes millions of quasars, which for the first time enables the realization of an optical reference frame at sub-milliarcsecond precision (median 0.4 mas), built solely on direct observations of extragalactic sources (Gaia Collaboration et al. 2018e). The Gaia-CRF2 is based on the positions of some 550000 quasars and has a substantial overlap with the radio ICRF, which allowed for the alignment of the optical reference frame to a prototype of ICRF3 at the 20-30 $\mu$ as level. The Gaia DR2 catalogue together with the Gaia-CRF2 thus represents a vast improvement for optical reference frames, providing mas-level positions to magnitude 21 for a dense network of sources all over the sky. This allows the astrometric anchoring of ongoing ground-based optical/IR surveys and will be essential for the operation of future extremely large telescopes.

\section{GLOBAL ASTROMETRY WITH THE HIPPARCOS/GAIA CONCEPT}

The concept for performing global astrometry with Gaia follows the same principles that were used for the Hipparcos mission (Lindegren 2005):

1. Collect observations simultaneously from two fields of view separated by a large angle.

2. Scan roughly along a great circle passing through both fields of view.

3. Make mainly one-dimensional measurements along the scanning direction.

\footnotetext{
${ }^{2}$ There are two important consequences of this change, often not realized by many of us: there is no epoch associated with the ICRS, and changes of source coordinates between different epochs can be calculated from the proper motions alone (where for rigorous epoch propagation the parallax and radial velocity are also needed, e.g. Butkevich \& Lindegren 2014).

$3^{3}$ http://hpiers.obspm.fr/icrs-pc/newww/icrf/index.php
} 


\section{Gaia in brief}

Gaia is the ESA space astrometry mission, launched in December 2013, collecting accurate positions, parallaxes, and proper motions for all sources to magnitude 20.7 in its white-light photometric band $G$ (covering the range $330-1050 \mathrm{~nm}$ ). Multi-colour photometry is obtained for all stars and radial velocities are collected for stars brighter than $G \approx 17$. Gaia's photometric instrument consists of two low-resolution fused-silica prisms dispersing the light entering the field of view. One disperser - called BP for Blue Photometer — operates in the wavelength range 330-680 nm; the other — called RP for Red Photometer — covers 640-1050 nm. From the integrated flux in the prism spectra two broad band blue and red magnitudes, $G_{\mathrm{BP}}$ and $G_{\mathrm{RP}}$, are defined. Radial velocities are measured with the Radial Velocity Spectrograph (RVS) which collects spectra over the wavelength range $847-874 \mathrm{~nm}$ at a resolution of $\sim 11000$. The apparent brightness of sources as measured by the integrated flux over this wavelength range is referred to as $G_{\mathrm{RVS}}$.

Gaia carries two telescopes with fields of view separated by $106.5^{\circ}$, of which the light is combined onto a single focal plane. Every six hours Gaia spins around the axis perpendicular the lines of sight of the telescopes. Sources observed by Gaia thus drift across the focal plane, and Time-Delayed Integration (TDI, or drift-scanning) is used to accumulate photo-electrons into a sharp image as the sources travel across a CCD. The wide dynamic range of Gaia is achieved by progressively reducing the integration time for bright sources $(G<13)$ through gates in the CCD detectors, which hold back and discard the photoelectrons accumulated before the gate (cf. Gaia Collaboration et al. 2016b). To fit the data collected by Gaia into the telemetry budget, only the pixels immediately around a source are read out and transmitted (cf. Figure 1). For bright sources the full 2D window is transmitted, while for fainter sources the window pixels are summed in the direction perpendicular to the scanning direction, leading to one-dimensional image profiles being transmitted to Earth.

DPAC: Gaia Data Processing and Analysis Consortium, tasked with turning the raw Gaia telemetry into the data releases.
4. The "basic angle" between the two fields must be known and extremely stable.

5. Repeat the measurements as many times as required to reach the desired astrometric accuracy, and with scans in varying orientations in order to cover the whole sky.

Below I summarize the motivations for this concept, the drivers of the astrometric precision, the basic elements of the astrometric data processing for Gaia, and the sources of systematic errors and how these might be mitigated. The focus is on explaining the concepts at a high level, but with sufficient detail such that anyone using the Gaia data has a basic understanding of how the astrometry is derived from the individual measurements, and what the main limitations are that should be considered when making investigations with Gaia catalogue data. Gaia Collaboration et al. (2016b) provide a detailed overview of the Gaia spacecraft, mission, and scientific instruments, and a summary of the entire DPAC data processing chain, including photometry, radial velocities, and higher level data products.

\subsection{Astrometry with Gaia}

Figure 1 illustrates how Gaia collects astrometric observables. The Gaia spacecraft consists of a three meter tall cylindrical structure, housing the service module and science instruments, which is kept in the shade by a ten meter diameter sunshield (see Fig. 1 in Gaia Collaboration et al. 2016b). This structure is schematically shown in Figure 1, top left, together with the spacecraft spin axis and the lines of sight of the two telescopes, at right 


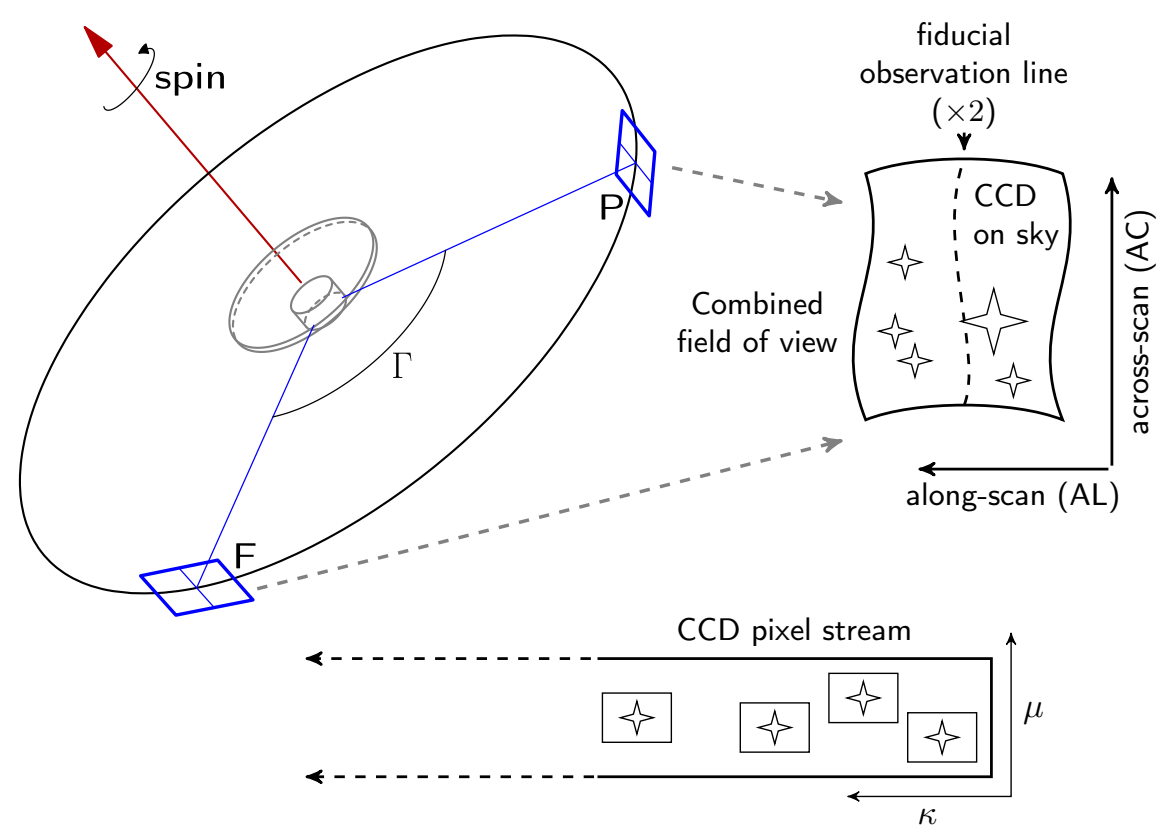

Figure 1

Astrometric observations with Gaia. At top left Gaia is schematically depicted, with the spacecraft spin axis pointing away from the sunshield, and the lines of sight of the two telescopes at right angles to the spin axis, separated by the basic angle $\Gamma$. The projection on the sky of the focal plane through both fields of view is indicated with $\mathrm{P}$ and $\mathrm{F}$. The top right illustrates schematically the collection of the basic observables $t_{\mathrm{obs}}$ (the observing time, the moment a source crosses the fiducial observation line) and $\mu_{\mathrm{obs}}$ (the AC location of the source). The bottom part of the figure shows the data stream in the form of continuously read out CCD pixels. Only the pixels in small windows around the sources are sent to the ground. The observables are derived from the source locations $\kappa(\mathrm{AL})$ and $\mu(\mathrm{AC})$ in this pixel stream.

angles to the spin axis and separated by the basic angle $\Gamma$. During one spacecraft revolution the fields of view of the telescopes scan along the indicated great circle. The light from the telescopes is imaged onto the same focal plane, covered with CCD detectors, and the projection on the sky of the focal plane through both fields of view is indicated with $\mathrm{P}$ for "preceding" and F for "following" in the sense of the spin direction of the spacecraft. The top right of Figure 1 schematically shows the projection on the sky of a CCD detector in the focal plane. The distortions indicate symbolically that details of the optical projection from telescope mirrors to the focal plane, in combination with the CCD location and orientation, as well as the properties of the CCD pixel grid, lead to a projected pixel grid that is not necessarily rectangular on the sky. This will be discussed further in the section on astrometric data processing. As the spacecraft spins the stars will drift from left to right across the field of view, in the so-called along-scan (AL) direction ${ }^{4}$ The perpendicular di-

\footnotetext{
${ }^{4}$ Note that stars drift along the focal plane in a direction opposite to the scanning direction dictated by the spacecraft spin vector. The stars drift from left to right along the CCD but the AL coordinate (equivalent to time) increases from right to left
} 
AL: Along-scan. Direction along the great circle scanned by Gaia's telescopes during one spacecraft revolution

AC: Across-scan:

Direction perpendicular to the along-scan direction.

$t_{\text {obs }}$ : Observation time of a source, derived from the centroid of the source image.

$\mu_{\mathrm{obs}}:$ AC location of a source at the time of observation $t_{\mathrm{obs}}$.

$\Omega$ : Azimuth of a source around the Gaia spin axis as defined in Figure 2

$\xi$ : Solar aspect angle. Fixed angle between Gaia's spin axis and the direction to the sun. rection is referred to as across-scan (AC). The basic observables are the times $t_{\mathrm{obs}}$ at which stars cross a fiducial observing line on the detector and the across-scan locations of the stars $\mu_{\mathrm{obs}}$ (both for each field of view). The observables are derived from the CCD image samples. To ensure the vast stream of pixel data fits in the telemetry budget, only the pixels immediately around a source image are transmitted, the so-called window (bottom part of Figure 1). In most cases the pixels in the window are summed on-board in the AC direction, leading to one dimensional profiles as source "images" (see Gaia Collaboration et al. 2016b, for the details on the focal plane read-out scheme). The observation times and across-scan locations are derived from the positions of the sources in these windows, together with information that allows placing the windows in the overall data stream.

Put simplistically, the observation times together with a knowledge of the spacecraft attitude (its orientation and spin phase) allow us to reconstruct the instantaneous celestial positions of the observed sources. Repeated measurement of the source positions then leads to determination of the parallax and proper motions. The actual estimation of the astrometric parameters is much more complicated as will be discussed in Section 3.3

The observing concept outlined above is based on the following considerations (Lindegren \& Bastian 2010. Lindegren 2005) for a space mission that implements in a single survey the traditional astrometric programmes (parallaxes, surveys, reference frames, Section 2.1).

3.1.1. Wide-angle measurements for reference frames and absolute parallaxes. The advantages of moving to space pointed out in Section 2.2 enable the bridging of large $(\sim 1$ radian) angles when measuring positions of sources with respect to each other. This is the only way to ensure that for any two sources separated by an angle $\rho$, the uncertainty on $\rho$ is independent of its value (Lindegren \& Bastian 2010). The astrometric catalogue is thus free of the zonal errors which result from the accumulation of errors when combining relative position measurements made in different parts of the sky over small angles. The presence of zonal errors makes for a less rigid astrometric reference frame and leads to systematic proper motion errors correlated over large scales, which could, for example, introduce erroneous interpretations of proper motions in terms of Milky Way dynamics.

Less intuitively, wide-angle astrometry enables the measurement of absolute parallaxes. This is schematically illustrated in Fig. 4 in Lindegren (2005) for a traditional parallax measurement, based on the observed angles between a target and a reference source, as having the reference source at 90 degrees from the target. For the specific case of Gaia the way parallax affects the measured positions (observing times) of sources is illustrated in Figure 2 The parallax shift $\boldsymbol{p}$ for any source (the difference between its direction as seen from the solar system barycentre and from the observer) is directed toward the solar system barycentre and is given as $p=\|\boldsymbol{p}\|=\varpi R \sin \theta$, where $\varpi$ is the parallax of the source and $R$ the distance from the observer to the solar system barycentre in au. What matters in the Gaia measurements is the parallax shift $p_{\|}$in the along-scan direction, which is $p_{\|}=p \sin \psi=\varpi R \sin \theta \sin \psi$ (see Figure 2). From the law of sines in spherical trigonometry it follows that $\sin \theta \sin \psi=\sin \xi \sin \Omega$, hence $p_{\|}=\varpi R \sin \xi \sin \Omega$.

Now consider two sources, one located in the preceding and one in the following field of view, with parallaxes $\varpi_{\mathrm{P}}$ and $\varpi_{\mathrm{F}}$ The large basic angle leads to large differences in the parallax factor $(\sin \xi \sin \Omega)$. In particular if the source in the preceding field of view is observed at $\Omega=0$, it will have zero parallax shift along scan, while for the other source the shift is $\varpi_{\mathrm{F}} R \sin \xi \sin \Gamma$. The situation is reversed if the source in the following field of view is at $\Omega=0$ (see Fig. 2 in Gaia Collaboration et al. 2016b). It is this property of 


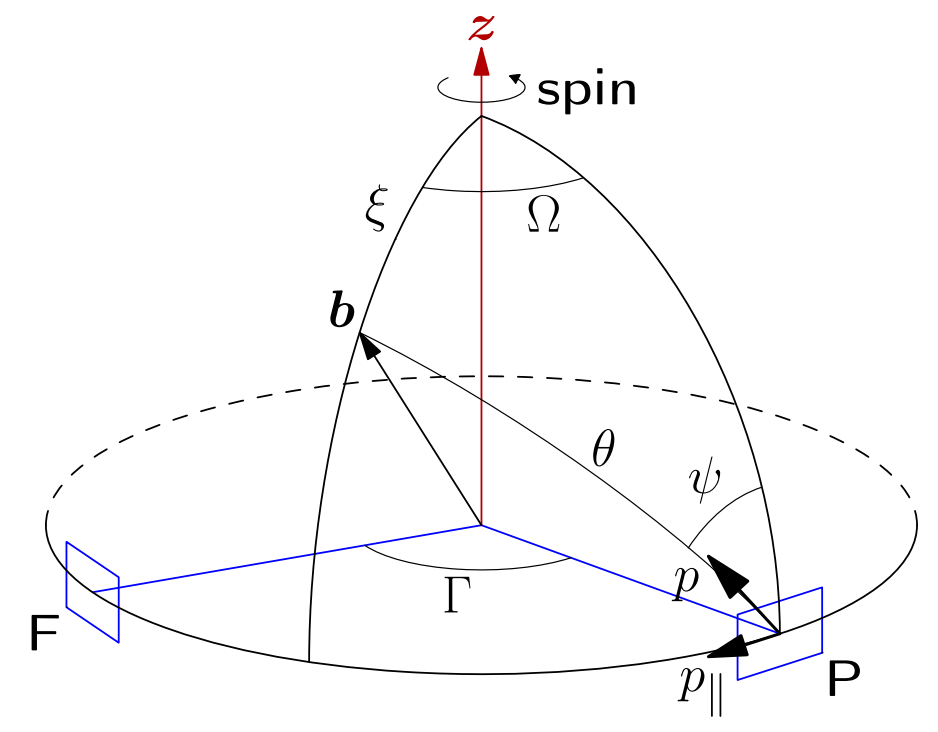

Figure 2

The parallax shift of a source as seen by Gaia. The spacecraft schematic is omitted here (cf. Figure 11. The source is seen in the preceding field of view which is at an azimuth $\Omega$ with respect to the meridian through the spacecraft spin axis $(\boldsymbol{z})$ and the direction to the solar system barycentre $(\boldsymbol{b})$. The angle between the source direction and $\boldsymbol{b}$ is $\theta$, while $\xi$ is the fixed angle between the spin axis and $\boldsymbol{b}$ (the "solar aspect angle"). The parallax shift of the source is toward $\boldsymbol{b}$ and proportional to $\sin \theta$. The vectors $\boldsymbol{p}$ and $\boldsymbol{p}_{\|}$indicate respectively the parallactic shift of the source and its projection on the along scan direction. Credits: Adapted from Lindegren 85 Bastian (2010), right panel of their Fig. 4 .

wide-angle astrometric measurements that allows us to disentangle the parallactic motions for different sources, meaning that the measurements are sensitive to each of $\varpi_{P}$ and $\varpi_{F}$. For narrow-field astrometry the parallax factors for all the stars in the field of view are nearly the same (as $\theta$ is nearly the same) and one is only sensitive to $\varpi_{\mathrm{P}}-\varpi_{\mathrm{F}}$, which makes additive corrections to the measured parallaxes necessary.

3.1.2. One-dimensional measurements. Ideally one would measure the actual angular separation between sources on the sky. However, when measuring sources simultaneously in two fields of view separated by a large angle (of order 90 degrees) it is sufficient to measure only the distance between the sources projected on the great circle passing through the two fields of view. As long as the fields of view themselves are relatively small (of order 1 degree) the difference between the projected and actual angular separation can be ignored (Lindegren 2005). Alternatively, this means that the measurement precision across-scan can be much lower (by a factor 100) than along-scan. This explains a number of aspects of the Gaia design: the smaller across-scan size of the Gaia telescope mirrors, the larger AC size of the CCD pixels, and the CCD read-out method (drift-scanning or time-delayed integration, TDI). The one-dimensional measurements also lead to simplifications in the calibrations (Section 3.3 and allow for significant savings in the amount of data to be sent down by numerically binning the CCD images in the AC direction. This also lowers the relative contribution of the read-out noise to the counts in the image samples. For the full 
design details refer to Gaia Collaboration et al. (2016b).

3.1.3. Scanning law. To see the parallactic and proper motions of the sources observed by Gaia it is necessary to measure them multiple times, with the scans intersecting at large angles in order to build up a rigid 2D network of angles between sources. The required continuous re-orientation of the spacecraft should be carried out as smoothly as possible and the full sky should be covered in a sufficiently short amount of time to allow for repeated scanning of the entire sky. The most efficient way found so far is to use the socalled uniform revolving scanning as implemented for both Hipparcos and Gaia (Lindegren \& Bastian 2010). The principle is that the spacecraft spins around the axis perpendicular to the two lines of sight, at a fixed rate $\left(60^{\prime \prime} \mathrm{s}^{-1}\right.$ for Gaia, i.e. a 6 -h spin period), with the telescopes thus scanning a great circle during one revolution. The spin-axis itself is made to precess around the direction to the sun, with a period of 63 days for Gaia, maintaining a fixed angle $\xi$ (Figure 2) between the spin-axis and the spacecraft-Sun direction. As the Sun moves along the ecliptic (as seen from Gaia) the spin-axis makes a slow looping motion around the direction to the sun. In combination with the spinning motion the full sky can be covered in about 3-4 months (see Figs. 6 and 7 in Gaia Collaboration et al. 2016b).

The parallax effect as seen by Gaia scales with $\sin \xi$ which would suggest $\xi=90^{\circ}$ as the best choice. However in this case the Sun would shine into the Gaia telescopes during every spacecraft revolution. In practice $\xi$ should be substantially less than 90 degrees, which follows from considerations on the size needed for the sunshield and the energy that can be collected by solar arrays mounted on the illuminated side of the sunshield (maximum for $\xi=0$ ). For Gaia the solar aspect angle is $\xi=45^{\circ}$. For more details on the Gaia scanning law see Gaia Collaboration et al. (2016b) and Lindegren \& Bastian (2010).

3.1.4. Basic angle. In order to conduct wide-angle astrometry the value of the basic angle should be large (of order 1 radian) and in principle the best value would be 90 degrees. However, while this is a good choice when a global astrometric solution is made (Section 3.3), in practice simple fractions $m / n$ of 360 degrees should be avoided. For Hipparcos this was motivated by the fact that the data processing proceeded in three steps (in order to keep the problem computationally tractable, Kovalevsky et al. 1992, Lindegren et al. 1992), where in the first step the star positions were reduced to coordinates along a great circle. If the relevant system of equations is examined it results that values of the basic angle that are simple fractions of 360 degrees lead to much higher variances on the derived positions (Lindegren \& Bastian 2010). While for Gaia enough computational power is available to avoid the data processing in steps, the so-called "First Look" astrometric solution still solves for star positions along a great circle in order to get quick (daily) and detailed insights into the health of the Gaia instruments (Fabricius et al. 2016, Jordan et al. 2005). Hence also for Gaia the basic angle value, $106.5^{\circ}$, was chosen to avoid simple fractions of $360^{\circ}$. It is essential that the basic angle is extremely stable in order to avoid an overall zero-point error on the parallaxes (Section 3.4. For Gaia stability at the few $\mu$ as level was required.

\subsection{Astrometric precision}

Before describing the data processing required to turn the Gaia measurements into an astrometric catalogue, I discuss here the basic drivers of the astrometric precision. Issues relating to accuracy are discussed in Section 3.4. Ultimately the astrometric precision 
depends on how well a source image can be located in the Gaia data stream. For the image location problem, Lindegren (2005) shows that the astrometric (i.e. angular) precision $\sigma$ scales as:

$$
\sigma \propto \frac{\lambda_{\text {eff }}}{B \sqrt{N}},
$$

where $\lambda$ is the effective wavelength of the measurements, $N$ the number of photons collected, and $B$ the aperture size of the mirrors (or the baseline of an interferometric system). This formula can be used to make basic design choices for a scanning astrometry mission. Typically a certain parallax accuracy is targeted, which scales as (cf. Section 3.1.1):

$$
\sigma_{\varpi} \propto \frac{\lambda_{\text {eff }}}{B \sqrt{N} \sin \xi} .
$$

The number of photons collected is proportional to: the average time spent observing a source, which is dictated by the field of view size as a fraction of the full sky, multiplied by the overall mission length; the photon collection efficiency of the system (driven by the telescope transmission and detector quantum efficiency); the photon flux received from a source; and the aperture area. The solid angle of the field of view is determined by the focal plane area and the telescope focal length. In the above formulae many complications are skipped over, such as the fact that multiple astrometric parameters as well as calibration information must be extracted form the same observations. Nevertheless the formulae allow making basic trade-offs in the design of a mission like Gaia. Cost drivers such as the value of $\xi$, aperture size, and focal plane size, can be compensated for by choosing a longer mission length. Lindegren (2005) describes how the enormous gains made from Hipparcos to Gaia can be understood from the basic precision scaling.

\subsection{Gaia Astrometric data processing}

Having reviewed the basic motivations for wide-angle astrometry from space and the basic design drivers for reaching a target accuracy, we can now turn to the question of how the astrometric parameters of the sources observed by Gaia are derived from the basic observables, $t_{\mathrm{obs}}$ and $\mu_{\mathrm{obs}}$ (Figure 1). The data processing problem for Gaia is formulated as a minimization problem (Lindegren et al. 2012, Eq. 1):

$$
\min _{\boldsymbol{s}, \boldsymbol{n}}\left\|\boldsymbol{f}^{\mathrm{obs}}-\boldsymbol{f}^{\mathrm{calc}}(\boldsymbol{s}, \boldsymbol{n})\right\|_{\mathcal{M}} \text {. }
$$

The task is to minimize the difference between the vector of observables $f^{\text {obs }}$ and their predicted values $\boldsymbol{f}^{\text {calc }}$. The latter depend on the source parameters $\boldsymbol{s}$ and a set of "nuisance parameters" $\boldsymbol{n}$, which have to be estimated along with the source parameters but are not themselves of interest. The norm in the equation above is calculated for a metric $\mathcal{M}$, where in practice for Gaia a weighted least squares solution is chosen taking the necessary precautions to make the solution robust.

Perhaps the easiest way to think about Equation 3 is to consider the forward problem of predicting where in the Gaia focal plane a source will be observed at a given moment in time. This involves four steps which are schematically depicted in the left panel of Figure 3 which is a simplified version of the schematic shown in Fig. 1 of Lindegren et al. (2012):

1. The source astrometric parameters $s$ determine the coordinate direction to the source at time $t$ as seen from the from the position of Gaia (i.e. the topocentric coordinate
Coordinate direction: Unit vector corresponding to the difference between the spatial coordinates of two locations (space-time events, strictly speaking).

Barycentric coordinate direction: The direction to a source as seen from the solar system barycentre, free from aberration and light bending effects.

Topocentric coordinate direction: Source direction as seen from the observer, thus including the parallax shift, but free from aberration and light bending effects.

Proper direction: The observable direction to a source as seen from the observer, including aberration and light bending effects. 

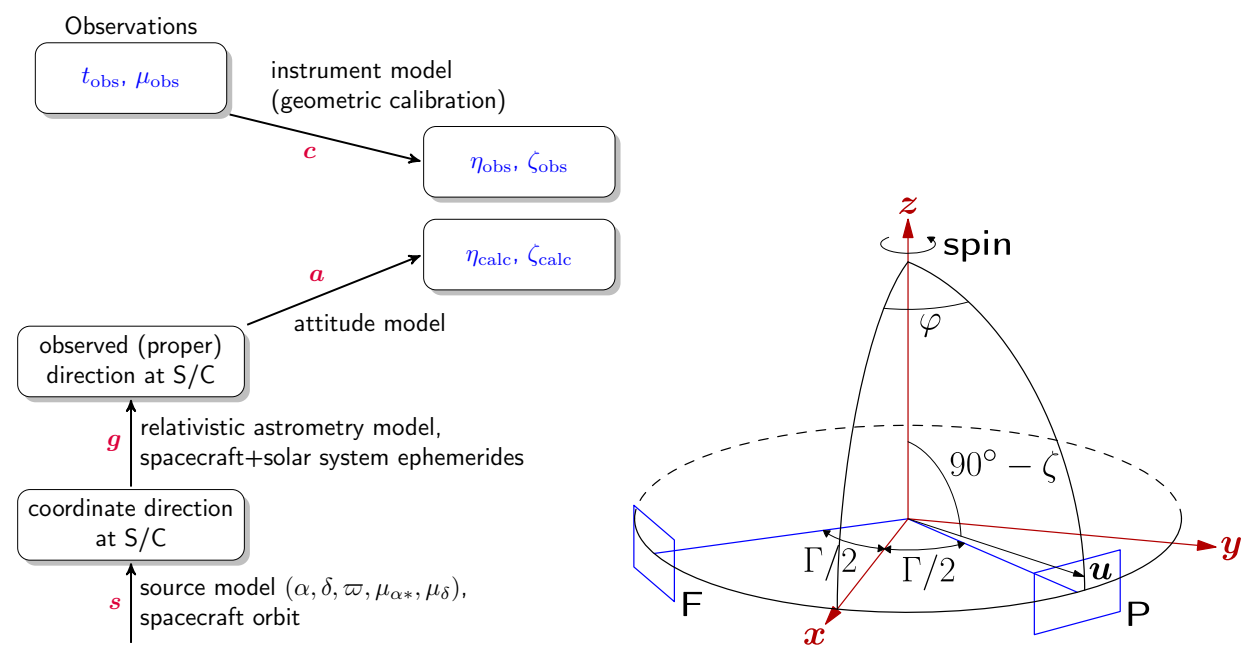

Figure 3

The left panel shows schematically how the astrometric observables collected by Gaia, $t_{\mathrm{obs}}$ and $\mu_{\mathrm{obs}}$, are modelled in terms of the field angles, $\eta$ and $\zeta$. The latter describe the (proper) direction $\boldsymbol{u}$ to a source as observed in the reference frame defined by the spacecraft axes $\boldsymbol{x}, \boldsymbol{y}, \boldsymbol{z}$, as shown in the right panel. For a source in the preceding field of view the angle $\eta$ is equal to $\varphi-\Gamma / 2$ (with $-\pi \leq \varphi<\pi$ ) while in the following field of view $\eta=\varphi+\Gamma / 2$. The angle $\zeta$ is defined as indicated in the figure. Credits: Right panel from Lindegren et al. (2012) (their Fig. 2), adapted with permission (C) ESO.

$\eta$ : Longitude-like angular coordinate of a source in a Gaia telescope field of view as defined in Figure 3

$\zeta$ : Latitude-like angular coordinate of a source in a Gaia telescope field of view as defined in Figure 3 direction $\overline{\boldsymbol{u}}$ ), in an axis system co-moving with Gaia but aligned with the ICRS (the so-called Centre of Mass Reference System or CoMRS, Lindegren et al. 2012, Klioner 2004). The effects of proper motion, radial velocity, and parallax are accounted for. The calculation of the parallax shift requires knowledge of the orbit of Gaia.

2. The source direction as seen from Gaia is affected by light bending by solar system objects and aberration due to Gaia's motion. These effects are included in this step to calculate the proper direction $\boldsymbol{u}$ to the source, still in the same axis system as above. The solar system ephemerides are required at this stage as well as the position and velocity of Gaia. A relativistic astrometry model is then applied to calculate the proper direction. The modelling is parametrized with a set of "global" parameters $\boldsymbol{g}$.

3. The source direction as seen from Gaia is now translated to a reference frame defined by the spacecraft axes (Figure 3 right panel). This involves a pure rotation from the ICRS to the spacecraft frame, which describes the orientation, or attitude, of Gaia. The attitude must be modelled from the observations and is described with the parameters $\boldsymbol{a}$. After this step the proper direction $\boldsymbol{u}$ is described in terms of the "field angles" $\eta$ and $\zeta$, which correspond to the along and across-scan directions (Figure 3 , where $\eta_{\text {calc }}$ and $\zeta_{\text {calc }}$ are the predicted field angles).

4. The final step is to convert the field angles into the actual location of the source in the focal plane. This involves among others the optical projection from the telescope field of view to the focal plane, the precise locations and orientations of the detectors, and the properties of their pixel grids. The parameters $c$ describing this step are referred to as the "geometric calibration" parameters (Lindegren et al. 2012). In practice the 
geometric calibration is used to derive "observed" field angles $\eta_{\mathrm{obs}}$ and $\zeta_{\mathrm{obs}}$ from $t_{\mathrm{obs}}$ and $\mu_{\text {obs }}$ (Figure 3 and Section 3.3.4.

When accounting for these steps Equation 3 can be expanded by replacing the nuisance parameters $\boldsymbol{n}$ with the specific parameter sets $\boldsymbol{a}, \boldsymbol{c}, \boldsymbol{g}$. Before summarizing the approach to solving this equation, some comments on the above elements are in order.

3.3.1. Source model. The first step above involves the modelling of the position of the source in the Barycentric Celestial Reference System (e.g., Klioner 2003), i.e. relative to the solar system barycentre, which allows predicting its barycentric coordinate direction. In the Gaia astrometric data processing all sources are treated as single objects moving with a uniform space velocity relative to the solar system barycentre (Eq. 3 in Lindegren et al. 2012). The source direction $\overline{\boldsymbol{u}}$ as seen from Gaia is calculated according to Eqs. 4-6 in Lindegren et al. (2012). The topocentric direction includes the parallax shift of the source as seen from Gaia which is given by the term $\varpi \boldsymbol{b}_{\mathrm{G}}(t) / A_{\mathrm{u}}$ in Eq. 4 of Lindegren et al. (2012), with $\boldsymbol{b}_{\mathrm{G}}(t)$ the barycentric position of Gaia and $A_{\mathrm{u}}$ the astronomical unit. This implies the need to know the orbit of Gaia. Solar system applications require the position to be known to better than $150 \mathrm{~m}$ such that main belt asteroid position uncertainties are not affected by uncertainties in the position of Gaia. Bright star aberration corrections require the velocity to be known to $2.5 \mathrm{~mm} \mathrm{~s}^{-1}$ (Section 3.3.2).

The above source model is strictly applicable only to single stars which are substantially smaller than 1 au in size. The presence of stellar or sub-stellar companions requires introducing the orbital motion of the sources in the model, while for very large (supergiant) stars the photocentre motion due to large convective cells in the atmosphere should be accounted for (Chiavassa et al. 2011, 2018). The latter complication is ignored in the Gaia data processing and can lead to less reliable astrometry for physically large stars. The presence of non-single stars cannot be ignored, but for determining the calibration parameters in the astrometric data processing $(\boldsymbol{a}, \boldsymbol{c}, \boldsymbol{g})$ only sources for which there is no evidence that they have a companion are used. The processing of non-single stars with a more complex source model is done in a separate, specialized process (Gaia Collaboration et al. 2016b, Sect. 7).

3.3.2. Relativistic astrometry model. The topocentric direction $\overline{\boldsymbol{u}}$ is transformed to the proper direction $\boldsymbol{u}$ (cf. Figure 3) using the prescriptions in Klioner (2003). To ensure that bright star astrometry is not affected by errors in the relativistic modelling of the apparent source directions, the light-bending and aberration effects should be predictable to $\sim 1 \mu$ as accuracy. As illustrated in Table 1 in Klioner (2003) this implies that the light bending effects of the Sun and all the major planets should be accounted for, as well as the effect of a number of the moons and asteroids in the solar system. This requires a precise knowledge of the ephemerides and masses of solar system bodies. To compute the aberration effects at the $1 \mu$ as level the velocity of Gaia should be known to $2.5 \mathrm{~mm} \mathrm{~s}^{-1}$. The solar system and spacecraft ephemerides are taken as known (they are not solved for from the observations). For Gaia DR1 and Gaia DR2 the INPOP10e solar system ephemeris was used (Fienga et al. 2013). The spacecraft orbit is obtained at the required level of accuracy through a combination of spacecraft tracking at the Gaia Mission Operations Centre, located at the European Space Operations Centre (ESOC) in Darmstadt, Germany, and optical observations of Gaia to determine its position in the plane of the sky (Altmann et al. 2014). More detail can be found in Gaia Collaboration et al. (2016b). 
The remaining aspects of the transformation from $\overline{\boldsymbol{u}}$ to $\boldsymbol{u}$ depend on a set of global parameters $\boldsymbol{g}$, which for example include the parametrized post-Newtonian parameter $\gamma$ describing the strength of light bending. The parameters $\boldsymbol{g}$ are normally considered known (e.g., $\gamma=1$ ) in the Gaia data processing but can in principle be solved for. This would allow tests of General Relativistic models, such as the determination of the value of $\gamma$ from Gaia observations. In particular the scanning law of Gaia has been optimized such that observations of bright stars near Jupiter are made a number of times during the mission. These data will allow an attempt at measuring the quadrupole moment of light deflection by Jupiter. How Gaia astrometry can be used in these and other tests of fundamental physics is discussed by Klioner (2014). Such tests will probably only be done in later phases of Gaia data processing operations, when the other parameter sets in the astrometric solution are sufficiently well known and understood.

For the Gaia EDR3 astrometric solution the set of global parameters was extended with a spin related instrument distortion model, aimed at suppressing parallax zero-point variations (Lindegren et al. 2020). This is discussed in Section 3.4.3

3.3.3. Attitude model. The attitude describes the orientation of the spacecraft frame (Figure 3 with respect to the CoMRS. The set of parameters $\boldsymbol{a}$ describes how the attitude varies as a function of time. Errors in the spacecraft attitude can translate into additional noise or errors on the source astrometric parameters (Section 3.4). To achieve the astrometric performance targeted for bright stars, the attitude reconstruction for Gaia should be accurate to the $10 \mu$ as level. The details of the attitude modelling are given in Lindegren et al. (2012). Risquez et al. (2013) present detailed simulations of the Gaia attitude as it evolves under the influence of solar radiation pressure torques, the noise in the micropropulsion system, and the effects of micro-meteoroid hits and minute structural changes in the spacecraft (the micro-clanks described in Gaia Collaboration et al. 2016b). They predict that the attitude knowledge will be limited to the $20 \mu$ as uncertainty level, which would add an additional $7 \mu$ as rms to the uncertainties of bright star parallaxes. As of Gaia DR2 the attitude modelling has not yet reached this accuracy level and it is one of the areas in which improvements are being made for future data releases.

3.3.4. Geometric calibration. The last step in the modelling of the astrometric observables is in practice done differently than implied in step 4 above (Lindegren et al. 2012). Rather than transforming the field angles to locations in the focal plane, the CCD pixels are instead projected into the space of field angles through the geometric calibration model with parameters $\boldsymbol{c}$. The source locations are measured as pixel coordinates (AL and AC, cf. Figure 1 in the data stream, translated to the observation time $t_{\mathrm{obs}}$ and the continuous across-scan coordinate $\mu_{\text {obs }}$. Because of the TDI observation mode, the measured locations must be referred to some observation time halfway through the time it takes to cross a CCD. The reference is taken to be a line of pixels halfway along the CCD, which is traced out in field angle space as the "observation line" $[\eta(\mu), \zeta(\mu)]$. Due to distortions in the optical projection from telescope field of view to focal plane, and the details of the CCD locations and orientations, as well as the pixel grids, the observation lines in general are not straight lines in $(\eta, \zeta)$, as depicted in Figure 1 and in Fig. 4 of Lindegren et al. (2012).

The geometric calibration model must accommodate effects on various spatial and time scales. Lindegren et al. (2012) discuss a division into large-scale calibration effects, such as thermal variations in the optics, the detectors and the mechanical support structures, 
and small-scale calibration effects, such as irregularities in the CCDs themselves. The TDI observation mode leads to a very significant simplification of the geometric calibration model in that any details in the CCD structure along scan (at the pixel level) are smeared out, such that only variations across-scan in the observation lines need to be modelled (with 4500 AL pixels this means a savings of a factor 4500 in calibration parameters). The large-scale calibration effects are modelled over relatively short timescales with low order polynomials in $\mu$. The small-scale effects are expected to be stable over longer timescales, but require detailed spatial modelling at the level of a few AC pixels. This translates into differing demands on the amounts of observations needed to calibrate a given effect. In particular it should be noted that the CCD gating, implemented to prevent the saturation of bright sources, introduces difficulties in that each gate represents a separate "instrument". The relative sparsity of bright sources makes the geometric calibration over short timescales more difficult for the different gate settings (a similar argument holds for the attitude calibration, as each gate sees a different effective attitude, Bastian \& Biermann 2005).

3.3.5. CCD pixel level data model. The time when a source was observed is derived by fitting the sample values of the image in the observation window around a source (cf. Figure 1) with a model that includes the total flux, local background level, and the image location $\kappa$, a continuous representation of the AL pixel coordinates (Lindegren et al. 2012). The samples are modelled with a point spread function (PSF), or line spread function (LSF) for the one-dimensional windows transmitted for the majority of sources. The image location is translated to observation time through a known relation between $t$ and $\kappa$. For a subset of sources the observation windows are two-dimensional which then also allows the derivation of the AC location $\mu$ (using a PSF model in this case).

The PSF/LSF model includes optical as well as electronic effects on the shape of the source image, and should also account for charge transfer inefficiency effects. The source colour, which one might have expected to be a part of the source parameters, is accounted for at this level through the effect of colour on the details of the PSF/LSF shape. Details on the PSF/LSF modelling as employed for Gaia EDR3 can be found in Rowell et al. (2020).

3.3.6. Solving the minimization problem. With the above components of the observation modelling for Gaia in mind, the model problem in Equation 3 can now be written as:

$$
\min _{\boldsymbol{s}, \boldsymbol{a}, \boldsymbol{c}, \boldsymbol{g}}\left\|\boldsymbol{f}^{\mathrm{obs}}\left(t_{\mathrm{obs}}, \mu_{\mathrm{obs}} \mid \boldsymbol{c}\right)-\boldsymbol{f}^{\mathrm{calc}}\left(t_{\mathrm{obs}} \mid \boldsymbol{s}, \boldsymbol{a}, \boldsymbol{g}\right)\right\|_{\mathcal{M}}
$$

which is a simplified formulation of Eqs. 24-26 in Lindegren et al. (2012). Solving this system of equations is done by splitting the problem into a "calibration" part (solving for $\boldsymbol{a}, \boldsymbol{c}, \boldsymbol{g}$ ) and a "source update" part (solving for $\boldsymbol{s}$ ). The calibration part is solved from a subset of "primary" Gaia sources, for which there is no evidence that they are non-single stars. Once the calibrations are solved the parameters of all Gaia sources are updated, keeping $\boldsymbol{a}, \boldsymbol{c}$, and $\boldsymbol{g}$ fixed. Lindegren et al. (2012) estimate that for $10^{8}$ primary sources $\sim 5.5 \times 10^{8}$ parameters have to be solved from $\sim 8 \times 10^{10}$ basic observables. Bombrun et al. (2010) showed that for these numbers a direct solution of the minimization problem was many orders of magnitude beyond computing capabilities in 2010. This is mainly due to the attitude and geometric calibration models linking together sources observed in the same field of view or at the same time in the two fields of view, leading to cross-terms between sources in the normal equations matrix over scales of $\sim 0.7^{\circ}$ and $\sim 106.5^{\circ}$. 
The solution chosen for the Gaia astrometric processing is the so-called Astrometric Global Iterative Solution (AGIS, Lindegren et al. 2012). The minimization problem in Equation 4 is tackled by iterating four partial solutions until convergence is achieved. Three of the four parameter sets are fixed and one solves for the remaining set. For example, assuming the source parameters are approximately known (from a starting catalogue or a previous iteration) together with the geometric calibration parameters (e.g., derived from the nominal instrument parameters), and taking the global parameters as given, one can solve for the attitude parameters. In subsequent iterations one solves for $\boldsymbol{c}, \boldsymbol{g}$, and $\boldsymbol{s}$, until convergence is achieved. Upon convergence, the parameters $\boldsymbol{a}, \boldsymbol{c}, \boldsymbol{g}$ as determined from the observations of the primary sources are then fixed and used to solve for the astrometric parameters of all sources.

Obviously this summary skips over many practical complications that require considerable and continuing efforts to solve. The details can be found in Lindegren et al. (2012), with specific updates and improvements that were implemented for Gaia DR1 and Gaia DR2 described in Lindegren et al. (2016) and Lindegren et al. (2018).

\subsection{Systematic errors in Gaia astrometry}

The scientific results from the analysis of the first two Gaia data releases very convincingly demonstrate the exquisite quality of the astrometric data, where in particular Gaia DR2 represents a major jump in the availability of high accuracy parallaxes and proper motions for large numbers of stars all over the sky. Nevertheless, Gaia astrometry is not and was not expected to be perfect and a number of works have demonstrated the presence of systematic errors in the parallaxes and proper motions, in particular the catalogue validation papers accompanying the data releases (Arenou et al. 2017, 2018). The systematic errors are very small, the global zero-point offset for Gaia DR2 parallaxes being 30-80 $\mu$ as. Nevertheless the systematics cannot be ignored. At the bright end of the Gaia survey the systematic errors should remain well below the random errors, which are at the tens of $\mu$ as level in Gaia DR2 for stars brighter than magnitude 12-14, to ensure the correct interpretation of, for example, subtle features in a Hertzsprung-Russell diagram. At the faint end of the survey there is the potential to measure a precise parallax for the Magellanic clouds by averaging over millions of stars, however this is currently limited by the systematic errors, as the parallaxes of these galaxies are in the 15-20 $\mu$ as range. The Gaia celestial positions and proper motions also exhibit systematic errors, in the form of regional patterns on the sky (such as seen in the maps of the astrometric parameters of quasars, see Fig. 11 in Gaia Collaboration et al. 2018e), and potentially in the form of a global misalignment and spin of the Gaia celestial reference frame with respect to the ICRF. In the following subsections the various types of systematic errors in Gaia astrometric data will be discussed, ending with a short discussion on how to characterize the systematics.

3.4.1. Global systematic errors: reference frame. The global astrometry concept ultimately rests on building a network of angles measured between sources on the sky. The angle between two sources on the celestial sphere is invariant with respect to the orientation or spin (change of orientation over time) of the sphere. Specifically for a Hipparcos/Gaiatype mission this means that an overall change in the positions of sources due to a change of the orientation of the celestial sphere is completely degenerate with a change in the spacecraft attitude (Butkevich et al. 2017). Hence, special measures have to be taken to 
align the orientation of the resulting reference frame to the ICRF, and to ensure that it does not exhibit an average spin with respect to the distant quasars. The principles of the construction of the celestial reference frame for Gaia DR2 (the Gaia-CRF2) are described in Lindegren et al. (2012, 2018) and its properties are described in Gaia Collaboration et al. (2018e). At $G=19$ the alignment of Gaia-CRF2 to the ICRF is believed to be accurate to $20 \mu \mathrm{as}$, while the frame is non-rotating at the $<20 \mu \mathrm{as} \mathrm{yr}^{-1}$ level (Gaia Collaboration et al. $2018 \mathrm{~b}$ e). The alignment of the Gaia reference frame and ensuring it shows no average spin, are done with a specially selected set of quasars (Lindegren et al. 2018, Gaia Collaboration et al. 2018e which are typically faint $(G>19)$ and have bluer colours than most stellar sources in the Gaia catalogue. Although the astrometric data processing for Gaia is set up to ensure a consistent solution across all source brightness and colour ranges, there are numerous factors that can cause inconsistencies between the reference frame at the faint and bright ends of the Gaia survey, in particular the abrupt change in the instrument configuration at $G=13$, where $2 \mathrm{D}$ instead of $1 \mathrm{D}$ observation windows are used and where measures against CCD saturation start to take effect (Lindegren 2020a). This is shown in Fig. 4 of Lindegren et al. (2018) where the bright stars (at $G<13$ ) show an average spin at the $0.1 \mathrm{mas} \mathrm{yr}^{-1}$ level compared to the quasars. The Gaia DR2 bright source reference frame was further investigated by Lindegren (2020a b) using VLBI observations of radio stars. The Gaia and VLBI astrometry of the radio stars were used to jointly estimate the orientation and spin parameters of the Gaia DR2 bright star reference frame, where the assumption was made that the VLBI astrometry is on the (non-rotating) ICRF. The earlier estimates of the spin were confirmed, while the value of VLBI observations of radio stars in characterizing the bright Gaia reference was demonstrated. It is stressed by Lindegren (2020a) that publications on VLBI astrometry should always provide the full astrometric solution as well as the epoch for the solution, where especially the celestial positions are of great historical value in reference frame investigations.

3.4.2. Global systematic errors: parallax zero-point. A second type of systematic that affects all sources equally is that of the global parallax zero-point. As explained in Section 2.2 the Hipparcos/Gaia concept enables the measurement of absolute parallaxes which means that in principle for a collection sources located at sufficiently large distances (such as quasars) the average measured parallax should be zero. It is well known that this is not the case for Gaia DR2 (Lindegren et al. 2018), the quasar parallaxes being $-29 \mu$ as on average, which by definition is the parallax zero-point. In addition the zero-point is known to exhibit variations as a function of (at least) source brightness, colour, and position on the sky. This was found both during the catalogue validation and in its first scientific use (see for example Figs. 12 and 13 in Arenou et al. (2018) and Fig. 16 in Gaia Collaboration et al. (2018c) for examples of position-dependent parallax zero-point variations), and in a number of subsequent investigations in which the parallax zero-point was estimated for different samples of stars (see for example Chan \& Bovy 2020, and references therein). The causes for the zero-point variations will be discussed in the next section, here we focus on the global parallax zero-point.

The astrometric parameters of a given source can be estimated thanks to the along and across-scan shifts in its position, measured at a series of epochs, with respect to the position expected at some reference epoch, normally the middle of the time interval covered by the mission. This is illustrated for the parallax in Figure 2 For the rest of this discussion we focus on the AL shifts which carry most of the astrometric information. Shifts of observed 


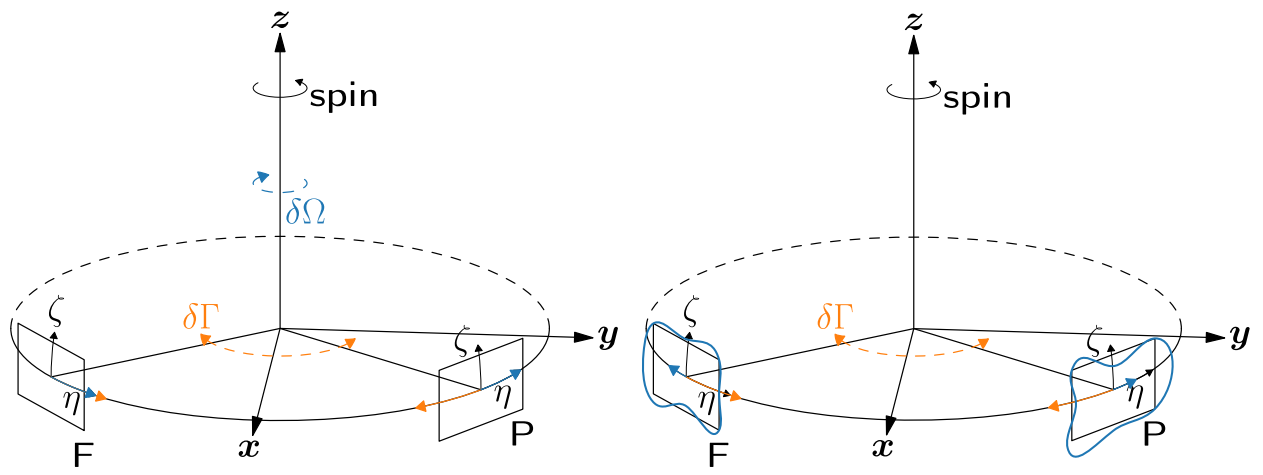

Figure 4

Illustrations of the degeneracy between source astrometric parameter changes and combinations of changes in the basic angle, spacecraft attitude, and geometric calibration. The left panel shows how an increase in the basic angle combined with a negative rotation of the spacecraft around $\boldsymbol{z}$ leads to changes in the apparent along-scan positions of a source (orange and blue vectors respectively). The right panel shows the same for a combination of an increase in the basic angle and changes in the focal plane projection on the sky (geometric calibration). Credits: Adapted with permission from figures in an unpublished technical note by S. Klioner.

along-scan source positions can be caused by other effects, such as physical changes in the spacecraft or the scientific instruments. Figure 4 illustrates three physical effects that can cause changes in the measured AL positions of sources. The left panel shows how an increase in the basic angle $\delta \Gamma$ leads to an AL shift of the source position to smaller values of $\eta$ in the preceding field of view and to larger values in the following field of view. The same panel also shows the effect of a small change in the spacecraft attitude caused by a small rotation $\delta \Omega$ around the $z$-axis, here in the sense opposite to the spacecraft spin. This leads to an increase in $\eta$ in both fields of view. The right panel in Figure 4 shows how a change in the details of the projection of the focal plane on the sky (due to evolving optics or thermoelastic changes in the focal plane structure, for example) can lead to effects in $\eta$ very similar to a basic angle increase. Errors in the spacecraft velocity components can also produce changes in the measured AL shifts, in this case due to apparent aberration effects moving the sources toward the direction of motion.

Comparing Figure 4 to Figure 2 qualitatively demonstrates how certain physical changes in the spacecraft and instruments cause apparent shifts in source positions as measured by Gaia. These apparent shifts could be confused for a real parallax effect and thus lead to systematic errors in the estimated parallaxes. This is developed rigorously for the case of basic angle and attitude changes by Butkevich et al. (2017). They show how certain combinations of basic angle and attitude changes are indistinguishable from a global parallax offset $\delta \varpi$ applied to all observed sources. One can develop the variations of the basic angle $\delta \Gamma$ as a general harmonic series in the spin phase $\Omega$ of the spacecraft with respect to the direction to the solar system barycentre (see Figure 2):

$$
\delta \Gamma=\sum_{k \geq 0} a_{k} \cos k \Omega+b_{k} \sin k \Omega,
$$

Butkevich et al. (2017) show that the $\cos \Omega$ component is completely degenerate with a global parallax offset (as well as specific attitude changes, see Eq. 17 in Butkevich et al. 
2017). The prediction is that in the presence of basic angle variations synchronous with the spacecraft spin period, the global parallax zero-point would amount to $\delta \varpi \sim 0.87 a_{1}$.

This degeneracy between basic angle variations and the parallax zero-point was already known in Hipparcos times (Butkevich et al. 2017). A major concern for both Hipparcos and Gaia was that the illumination of spacecraft by the sun also varies with the spin period (due to lack of complete symmetry of the spacecraft structures about the spin axis), which could thus lead to spin-synchronous basic angle variations and hence parallax zero-point offsets. To ensure the targeted parallax accuracies for Gaia at the bright end, the basic angle was thus required to be stable to the few $\mu$ as level over the spacecraft spin period (Gaia Collaboration et al. 2016b). This was not achieved in practice, with the actual basic angle fluctuations being much larger at the $\sim 1$ mas leve 5 including a prominent 6 -h period component which is strongly coupled to the spin phase $\Omega$ of the spacecraft (Lindegren et al. 2016). If left uncorrected these basic angle variations would thus lead to a $\sim 0.87$ mas parallax zero-point for the Gaia data releases. Because it was anticipated that maintaining the basic angle stable at a few $\mu$ as would be extremely challenging, a metrology device capable of measuring the basic angle changes was developed and prototyped early on in the Gaia project (Meijer et al. 2009, Gielesen et al. 2013, Mora et al. 2014). This so-called Basic Angle Monitor on board Gaia can measure the basic angle changes to a few $\mu$ as precision over short timescales of 10-15 min (Gaia Collaboration et al. 2016b). This information on the basic angle fluctuations was used in the astrometric data processing for both Gaia DR1 and DR2 to correct for the effects on the parallax estimation (Lindegren et al. 2016, 2018). Despite the corrections based on the Basic Angle Monitor an overall parallax zero-point nevertheless remains in the first two Gaia data releases. This is partly because the monitor measures the basic angle variations as seen from a specific part of the Gaia focal plane (see Fig. 4 in Gaia Collaboration et al. 2016b), which means that the metrology data is not fully representative of the effective basic angle variations seen over the rest of the focal plane.

Butkevich et al. (2017) point out that the degeneracy between parallax and the 6 -h basic angle variations is not perfect. Three factors contribute to slightly breaking the degeneracy: the finite size of the telescope fields of view causes slight variations in the parallax shift that can be distinguished from basic angle variation effects; the Sun as a driver of the basic angle fluctuations leads to a basic angle varying with the inverse square distance between Gaia and the Sun (which changes over the course of a year); for the same reason the basic angle varies not with the spin phase of the spacecraft with respect to the direction to the solar system barycentre, but with the spin-phase referred to the slightly different direction to the sun. Thus in principle there is a handle in the Gaia measurements to self-calibrate the effect of the $\cos \Omega$ component of the basic angle fluctuations and remove the parallax zero-point offset. For reasons discussed in the next section this is very non-trivial and is an area of active research and development for future Gaia data releases. Finally, it should be stressed that basic angle fluctuations on timescales longer than the 6-h spin period can be calibrated as part of the astrometric data processing and are included in the geometric calibration parameters $\boldsymbol{c}$ from Section 3.3

3.4.3. Zonal errors. Butkevich et al. (2017) note that the basic data processing approach for Gaia, as captured in Equation 4, contains many potential degeneracies, of which they

\footnotetext{
${ }^{5}$ Note that mas-level basic angle fluctuations amount to only tens of nm sized movements on the physical scale of Gaia.
} 
discuss in detail the degeneracy between $\boldsymbol{s}$ (which includes the parallax), attitude $\boldsymbol{a}$, and the basic angle component of $\boldsymbol{c}$. In principle degeneracies between parameters in the astrometric solution are not a problem as long they concern nuisance parameters only. For example a degeneracy between subsets of $\boldsymbol{a}$ and $\boldsymbol{c}$ would lead to errors in the interpretation of the attitude and geometric calibration but this would be harmless for the parameters $s$ of interest. However in practice there are degeneracies between all four parameter groups, meaning in particular that changes in combinations of $\boldsymbol{a}, \boldsymbol{c}$, or $\boldsymbol{g}$ may be misinterpreted as changes in $\boldsymbol{s}$, where it should be stressed that positions and proper motions may also be affected. The effects on $s$ of these complex degeneracies are imprinted on the celestial sphere after filtering through the Gaia scanning law and this could be an explanation for the regular patterns seen in, for example, the median parallaxes and proper motions of stars in the LMC (Arenou et al. 2018, Gaia Collaboration et al. 2018c). The way this works can qualitatively be understood as follow: ${ }^{6}$ The attitude and geometric calibration of the spacecraft both vary as a function of time. The attitude is continuously forced to vary as part of the scanning of the sky (and can undergo short-timescale changes due to micro-meteoroid hits for example), while the geometric calibration varies as the optics evolve and the Gaia instruments undergo thermoelastic changes, for example driven by solar illumination changes and instrument aging. Now suppose that particular combinations of errors in $\boldsymbol{a}$ and $\boldsymbol{c}$ are indistinguishable from certain changes to $\boldsymbol{s}$. If these errors in $\boldsymbol{a}$ and $\boldsymbol{c}$ evolve over time, the scan law will transform these to changes in $s$ as a function of position on the celestial sphere, which could thus lead to an imprint of specific systematic error patterns. This also implies that these patterns will occur all over the sky. In practice they are easily visible only in areas such as the LMC due to the high density of stars with similar parallaxes and proper motions. The size scale of these patterns can be expected to be of the order of the Gaia field of view AC $\left(0.7^{\circ}\right)$ but could be as small as the AC height of a CCD projected on the sky $\left(\sim 0.1^{\circ}\right)$.

A detailed understanding of how degeneracies between $\boldsymbol{s}, \boldsymbol{a}, \boldsymbol{c}$, and $\boldsymbol{g}$ translate to zonal errors may lead to specific strategies to handle the calibrations within AGIS in such a way as to suppress or mitigate these errors. This has been the focus of a considerable research effort within the DPAC over the past decade. For the Gaia EDR3 astrometric solution a first version of a model that can deal with spin-related instrument distortions on short timescales (less than a few Gaia spin periods) has been successfully implemented. This model, the parameters of which are included in $\boldsymbol{g}$, is summarized in Lindegren et al. (2020), who show that indeed the zonal parallax and proper motion errors are significantly reduced compared to Gaia DR2. By capitalizing on the above mention effects that remove the complete degeneracy between basic angle variation and the parallax zero-point, the model was also used to correct the parallax zero-point to a value closer to zero for Gaia EDR3.

3.4.4. Brightness and colour-dependent systematic errors. The systematic errors (as well as the uncertainties) in the Gaia astrometry show clear dependencies on source apparent magnitude and colour (Arenou et al. 2018, Lindegren et al. 2018). The magnitude dependencies are driven by the instrument configuration changes as a function of source brightness (Gaia Collaboration et al. 2016b). For sources brighter than $G=13$ two-dimensional observation windows are used in combination with CCD gates to prevent image saturation. For

${ }^{6}$ Based on a detailed elaboration in unpublished DPAC technical notes by T. Hilger and S. Klioner. 
sources fainter than $G=13$ one-dimensional windows are used, where at $G=16$ a switch to shorter $1 \mathrm{D}$ windows occurs. By using gates the integration time is reduced for bright stars (see the box on Gaia). This means that a slightly different effective spacecraft attitude is seen for these observations (Bastian \& Biermann 2005), but also that the PSF and geometric calibrations are slightly different. For the $2 \mathrm{D}$ windows a full PSF model is employed in the image location, while for the 1D windows a LSF is used. Hence each window/gate combination effectively introduces a new "instrument" to calibrate. The number of such "instruments" grows in particular at the bright end where the number of calibration sources becomes smaller and more sparsely distributed on the sky. This makes the estimation of $\boldsymbol{a}$ and $\boldsymbol{c}$ more vulnerable to systematic errors. In addition the high signal to noise ratio in the source images makes the image location process more sensitive to PSF modelling deficiencies. The changes in the instrument configuration related to gates and observation windows are clearly visible in the astrometric uncertainty distribution shown in Lindegren et al. (2018) and also in the photometric uncertainties shown in Evans et al. (2018). In both cases the most prominent effect is that for sources brighter than $G=13$ there is a clear "calibration floor" in the uncertainties, where systematic errors dominate.

The colour dependencies are introduced to a large extent by deficiencies in the PSF and LSF models. The precise shape of a source image in the focal plane depends also on its colour and this must be taken into account both in the calibration of the PSF/LSF models and in their application, when estimating the image location to obtain $t_{\text {obs }}$ and $\mu_{\text {obs }}$ Rowell et al. 2020). For Gaia DR1 and DR2 the PSF/LSF models were assumed to be independent of colour and time, leading to systematically biased image locations for sources that did not have a colour matching the effective colour of the PSF/LSF model. This was handled for Gaia DR2 by adding terms in the astrometric solution that can model the image location shifts as a function of time, colour, and magnitude (Lindegren et al. 2018), but this cannot completely prevent the occurrence of colour-dependent systematics. The assumption of a $\mathrm{PSF} / \mathrm{LSF}$ that is constant in time is a poor approximation to the actual evolution of the Gaia image characteristics, driven by thermal relaxation of the payload as well as the effects of contamination early in the mission (Gaia Collaboration et al. 2016b). Thus the image location systematics will also evolve with time which can lead to further zonal effects on the sky after filtering through the scan law. There are numerous other effects that complicate the image location process and introduce further systematic errors. These include the estimation of the local sky background, knowledge of the source colours (which depends on the photometric data processing for Gaia), and charge transfer inefficiency effects on the PSF/LSF (which can be ignored for the early Gaia data releases, but grow over time). See Rowell et al. 2020) for more details on the PSF/LSF modelling.

The challenge for upcoming Gaia data releases is to suppress as much as possible the systematic errors (global and zonal), such that they will not start dominating the overall accuracy budget of the increasingly precise Gaia data (thanks to a longer period of measurement). In particular in the areas of self-calibration of the basic angle variations, the removal of zonal errors, and the improved modelling of the PSF/LSF, major efforts are being undertaken within the DPAC. Significant improvements have already been achieved for Gaia EDR3 as described in Lindegren et al. (2020).

3.4.5. Characterization of systematic errors. The unprecedented quality of the Gaia data releases as well as their all-sky coverage at high angular resolution make the assessment of the systematic errors a very challenging task. The characterization of the systematic 
errors in the Gaia astrometry is done at several levels during the data processing, both internally to the astrometric solution (Lindegren et al. 2016, 2018), and at the level of the catalogue validation (Arenou et al. 2017, 2018). Good internal checks on systematic errors are the comparisons of astrometric solutions constructed for different subsets of the observations (to get a handle on the variance in the zonal errors) and the examination of the parallaxes and proper motions derived for quasars (to understand the uncertainty statistics, the global parallax zero-point, and the reference frame quality). External checks are harder to come by as there is only very limited astrometric data that is of comparable quality to Gaia. Nevertheless numerous studies have been undertaken to estimate the parallax zero-point for different subsets of sources where one can have confidence in independent distance estimates (see examples in Arenou et al. 2018), or where a good understanding of the astrophysics of the source samples allows for joint estimations of the parallax zero-point and intrinsic source properties. Examples include the use of Cepheid and RR Lyrae variables (e.g., Sesar et al. 2017, Riess et al. 2018) and Red Clump stars (possibly in combination with asteroseismological data, e.g., Chan \& Bovy 2020, Hall et al. 2019, Khan et al. 2019, Zinn et al. 2019). These studies have confirmed the magnitude, colour and, sky position dependencies of the parallax zero-point. The complex and difficult-to-characterize variations in the zero-point are the main motivation to leave the astrometry results from the Gaia data processing uncorrected upon publication.

Ultimately the insights into the systematic errors in the Gaia data releases will be driven by the continued scientific exploration of the data, demanding consistency between the data and the astrophysical picture of the Milky Way. A specific example is the study by Schönrich et al. (2019), which explicitly demands consistency between the astrometric data and the spatial and velocity distribution of the stars in the subset of Gaia DR2 for which radial velocities were measured. They estimated a parallax zero-point consistent with other estimates for the bright end of Gaia DR2, and identified an unexplained correlation between the parallax zero-point offset and the parallax uncertainty.

\section{GAIA SCIENCE HIGHLIGHTS}

\subsection{Revolutionizing science from the solar system to the distant universe}

The publication of Gaia DR1 (Gaia Collaboration et al. 2016a) marked the start of a new era in the availability of fundamental astronomical data. Parallaxes and proper motions were provided for 2 million sources, reaching sub-milliarcsecond accuracies and providing a first taste of absolute microarcsecond astrometry in the optical for the proper motions of the subset of Hipparcos stars ( $60 \mu \mathrm{as} \mathrm{yr}^{-1}$ average uncertainties). In addition an unprecedented map of the sky was provided, with positions at the milliarcsecond accuracy level for 1.1 billion sources out to 20th magnitude. This vastly improved astrometric reference was used to anchor other surveys. This led to the production of large and accurate proper motion catalogues by combining Gaia DR1 and older sky surveys (e.g. Altmann et al. 2017, Deason et al. 2017, Tian et al. 2017, Zacharias et al. 2017). However, Gaia DR2 (Gaia Collaboration et al. 2018b) represents a more profound transformation of astronomy, providing: a high precision sky map for 1.7 billion sources at an angular resolution significantly exceeding that of ground-based large-area optical sky surveys; microarcsecond to milliarcsecond quality parallaxes and proper motions for 1.3 billion sources out to the survey limit at $G \sim 21$ (Lindegren et al. 2018); the first realization of the optical celestial reference frame constructed on the basis of extragalactic sources only (Gaia Collaboration et al. 2018e); an 
all sky homogeneous multi-colour photometric survey (Evans et al. 2018, Riello et al. 2018); and a large radial velocity survey of some 7 million sources out to $G \sim 12$ (Katz et al. 2019 , Sartoretti et al. 2018). In addition Gaia DR2 provides light curves and properties for 550000 variable stars (Holl et al. 2018, Gaia Collaboration et al. 2019), astrophysical parameters (effective temperature, extinction, radius, and luminosity) for some 160 million sources (Andrae et al. 2018), and a first catalogue of astrometry of minor bodies in the solar system (Gaia Collaboration et al. 2018f).

As of September 2020 the papers describing Gaia DR1 and DR2 collectively were cited $\sim 6400$ times 7 , which gives a first impression of the enormous impact of the Gaia data releases. The data is being used in many different ways, ranging from looking up the parallax, proper motion, photometry, or radial velocity for a specific source, to analyses of large subsets of the Gaia data, or even the full data set. The ready availability of fundamental astronomical data for 1.7 billion sources has quickly made Gaia DR2 and indispensable part of astronomical research today, to the point of making it hard to imagine a time when this data was not available. In the following subsections a highly selected overview of science results from Gaia will be given, starting in the solar system and going at all the way to the distant quasars. Early science highlights from Gaia DR1 can be found in Brown (2018) and the corresponding proceedings of the first IAU Symposium on Gaia results held in 2017 (Recio-Blanco et al. 2018).

Two decades ago Freeman \& Bland-Hawthorn (2002) introduced the field of Galactic Archaeology, the unravelling of the formation history of the Milky Way by using the combination of accurate phase space and chemical composition data for vast numbers of stars. They predicted that the field would be revolutionized by the Gaia mission data. We have now arrived at this point, as demonstrated in the review by Helmi (2020) on the insights provided by Gaia on the early evolution of the Milky Way.

4.1.1. Solar system. Gaia autonomously decides which sources to observe, in principle the only criteria being that a source is brighter than the survey limit $(G \sim 20.7)$ and point-like (de Bruijne et al. 2015, Gaia Collaboration et al. 2016b). Minor bodies in the solar system, such as main belt asteroids, near-Earth objects, and Kuiper-belt objects (referred to as "solar system objects" in the following), move with median speeds of about 7 mas s$^{-1}$ relative to stars in Gaia's along-scan direction (Tanga \& Mignard 2012). During the detection stage the images of these objects will thus be smeared slightly (by a fraction of a pixel) which in general leaves them sufficiently point-like that they will be measured by Gaia. Extrapolating from the asteroid population known at the time, Tanga \& Mignard (2012) estimated that about 350000 solar system objects will be observed by Gaia, including a few moons of major planets. Gaia's exquisite astrometry combined with its spectrophotometric data will revolutionize studies of solar system objects through the combination of high precision orbital and taxonomic classifications (Delbo' et al.2012). Gaia DR2 provides a foretaste in the form of astrometry and $G$-band photometry for a sample of about 14000 solar system objects. As shown in Gaia Collaboration et al. (2018f), the astrometry is already at the level where uncertainties in the individual position measurements range from 1 to 10 mas. This represents an improvement by two orders of magnitude over the average astrometry available in the archives (Desmars et al. 2013, find average residuals of $\sim 400$ mas for orbital fitting of the (relative) CCD astrometry collected by Minor Planet Center). For a subset of

\footnotetext{
${ }^{7}$ As counted on September 112020 using https://ui.adsabs.harvard.edu/
} 
the solar system objects just the 22 months of data collected for Gaia DR2 enable estimates of the orbital parameters comparable in quality to what can be obtained from ground based data collected over many decades (Fig. 32 in Gaia Collaboration et al. 2018f).

However, the full exploitation of the Gaia epoch astrometry of asteroids is possible only by coupling it to the available archive data. This is strictly required to measure subtle secular effects, important to understanding the dynamical evolution of the asteroid belt, such as the Yarkovsky acceleration due to the recoil of the emission of thermal photons. The large disparity in accuracy between space- and ground-based astrometry necessitated implementation of a very sophisticated weighting scheme (Spoto et al. 2019), and a totally new approach to mitigating the effect of zonal and regional errors (Tanga et al. 2020) in pre-Gaia stellar catalogues.

The main impact of Gaia DR1 and Gaia DR2 on solar system science has been the availability of a high accuracy all sky map of star positions, parallaxes, and proper motions. This enables much more precise predictions of occultations of stars by solar system objects (Desmars et al. 2019b), greatly enhancing the success rate and scientific value of occultation campaigns. Orbits of solar system objects are better determined by recalibrating the existing astrometry against the Gaia catalogue. Spoto et al. (2017) showed that orbit predictions from observations made over short time intervals can be much improved already with Gaia DR1, increasing the reliability of hazard predictions for near-Earth objects. The quality of the astrometry derived from past occultations of Gaia stars improves to a level that several $(\sim 10)$ good occultation observations are by themselves sufficient for orbit determinations with accuracies comparable to those based on decades of existing measurements.

Desmars et al. (2019a) fitted light curve data from past occultation campaigns to derive precise astrometric positions of Pluto with respect to the Gaia DR2 positions of the occulted stars. The resulting improved ephemeris for Pluto is precise to the milliarcsecond level for the period 2000-2020 (a precision not attainable with direct astrometry of Pluto itself, Desmars et al. 2019a), which allows for better predictions of future occultations. The light curve modelling must account for refraction effects in Pluto's atmosphere, from which one can infer properties of the atmosphere (temperature and pressure profiles). Meza et al. (2019) used occultation data from 2002-2016 to infer an increase in Pluto's atmospheric pressure over the period 1988-2016. This finding is based on a model that simulates volatile $\left(\mathrm{N}_{2}\right)$ cycles over seasonal and astronomical timescales, and takes into account topography data from the New Horizons mission, including the location of large ice reservoirs. The pressure increase is explained by the enhanced heating of nitrogen ice at the onset of Pluto's northern spring. The pressure was predicted to peak within the next few years and then drop as the solar insolation decreases (due to Pluto's increasing distance to the sun) and nitrogen condenses onto Pluto's colder southern regions. Arimatsu et al. (2020) indeed present evidence for a drop in pressure based on an occultation observation from 2019.

The prediction of Pluto's shadow trajectory on Earth for the July 192016 occultation was improved thanks to the pre-release of the Gaia DR1 position of one star. Gaia DR2 astrometry was pre-released to support the observations of the occultation of a star by Triton on October 52017 (Marques Oliveira et al. 2018), leading to successful observations of the central flash in the light curve. The latter enables studies of the atmospheric temperature profile of Triton down to low altitudes (Marques Oliveira et al. 2019).

Another exciting application concerns the observations of occultations by the Centaur 10199 Chariklo, the only known minor planet with rings. By combining the observed 
occultation of 5 stars in Gaia DR1, Leiva et al. (2017) were able to derive plausible shape models for Chariklo, along with precise volume and density estimates. The accuracy of the occultations also provides a hint of topographic features of a size comparable to those of the icy moons of Saturn. Based on the total derived mass of the object, the rings appear to fall in the 3:1 mean motion resonance with respect to the rotation period of Chariklo.

The above offers a dramatic illustration of the power of an accurate sky map to faint magnitudes, which enables the monitoring of the atmospheric evolution of small bodies at billions of kilometers from Earth. A possibly even more impressive illustration is the support that was offered to the extended New Horizons mission which was to visit the Kuiper Belt object Arrokoth after its visit to Pluto. Pre-release Gaia DR2 astrometry was provided to the New Horizons team in order to refine the orbit determination for Arrokoth, through a more accurate astrometric calibration of HST imaging of this object (for which parallaxes and proper motions were required, Buie et al. 2020, Porter et al. 2018). The improved orbit was essential to the navigation of the New Horizons spacecraft. In addition the pre-release astrometry was used to plan the observation of four stellar occultations by Arrokoth. Analysis of the occultation data showed that Arrokoth is a contact binary with a $10 \%$ albedo (compare Fig. 10 of Buie et al. (2020) to Fig. 1 of Stern et al. (2019)), and allowed to further refine its astrometry and orbit. The results were used in the detailed planning of the Arrokoth flyby. Buie et al. (2020) and Porter et al. (2018) emphasize that the availability of Gaia DR2 astrometry enables much more precise predictions of stellar occultations by Kuiper Belt objects, well in advance of the events so that large-scale observation campaigns can be organized. This opens up many opportunities for obtaining shape measurement of Kuiper Belt Objects at resolutions of $1 \mathrm{~km}$ or better (Buie et al. 2020), and for getting upper limits on the presence of tenuous KBO atmospheres.

Other space missions that currently profit from stellar occultations for accurate planning and target characterisation are LUCY (NASA), which will explore the asteroid Trojans of Jupiter (Noll et al. 2020), and DESTINY+ (JAXA) planned to visit 3200 Phaethon Arai et al. 2020). The occultation campaign for this asteroid of only $\sim 6 \mathrm{~km}$ in size, very challenging due to extremely high requirements put on the predictions, was the first one to be successful for a member of the near-Earth object category.

4.1.2. Exoplanets and proto-planetary disks. Gaia will eventually detect planets astrometrically through an analysis of the epoch astrometry in cases where the simple single star source model does not fit the data (Gaia Collaboration et al. 2016b). Perryman et al. (2014) predicted that some 21000 exoplanets with masses down to $\sim 1-15 M_{\mathrm{J}}$ should be discovered out to $\sim 500$ pc during the nominal five-year Gaia mission, and up to 70000 if the mission lifetime is doubled to ten years. However this requires the collection of astrometric data over a sufficient time interval and very well understood astrometric uncertainties at the level of individual position measurements. Hence exoplanet catalogs are only expected for later Gaia releases. Nevertheless Gaia DR2 significantly impacted the field of exoplanet studies through the better characterization of exoplanet host stars, and studies of the discrepancies between long time baseline proper motions derived from combining Hipparcos and Gaia positions, and the proper motions measured by Hipparcos or Gaia alone.

The precise estimation of exoplanet properties such as radius, mass, and density, require precise knowledge of the properties of the host star, such as its radius and evolutionary state. Gaia DR2 lists radii and luminosities for a subset of the stars in the catalogue Andrae et al. 2018), while independently Fulton \& Petigura (2018) and Berger et al. (2018) used the 
Gaia DR2 parallaxes to derive precise (5-8\%) estimates of radii of stars in the Kepler field. This allowed, for example, to confirm and better characterize the presence of a gap around $2 R_{\oplus}$ in the size distribution of small close-in planets (Berger et al. 2018, Fulton \& Petigura 2018). Berger et al. (2020b) presented a refined catalogue of parameters for $186301 \mathrm{Kepler}$ stars with median radius accuracies of $4 \%$ and including more stellar parameters, such as ages and densities. This allowed Berger et al. (2020a) to make a more refined study of the planet radius gap as a function of stellar mass and age, finding hints in the ratio of super-earths to sub-neptunes as a function of host age, that core powered mass loss may be the driving mechanism for the radius gap. They caution that the sample is as yet too small to rule out the photo-evaporation mechanism. In another application of the Gaia data, advantage is taken of the high resolution sky map to properly interpret Kepler and TESS light curves, which may be affected by blending of several sources into the large PSFs and detector pixels used by these missions (e.g., Cloutier et al. 2020).

Combining Gaia and Hipparcos data allows for the derivation of proper motions over $\mathrm{a} \sim 24 \mathrm{yr}$ baseline. The long-term proper motions can be compared to the Hipparcos and Gaia DR2 proper motion measurements which cover baselines of $\sim 3.5$ yr and 22 months, respectively. Discrepancies between the three measurements can point to the presence of unseen companions orbiting the target stars, including sub-stellar objects and exoplanets (Mignard 2009, Michalik et al. 2014). Snellen \& Brown (2018) used the proper motion discrepancy to constrain the orbital period and mass of the young planet $\beta$ Pictoris $b$. They took advantage of the edge-on orbit of the planet which tightly constrains the expected direction of the stellar reflex motion. A planet mass of $11 \pm 2 M_{\mathrm{J}}$ was derived which provides support for so-called hot-start models for gas giant formation. The mass of $\beta$ Pic b was confirmed in studies by Dupuy et al. (2019) and Nielsen et al. (2020), in which radial velocities and high angular resolution imaging data were added to the analysis of the proper motion discrepancy. Brandt (2018) applied the proper motion discrepancy technique to the full Hipparcos Catalogue, and derived a catalogue of Hipparcos-Gaia DR2 accelerations aimed at facilitating a search for sub-stellar and dark companions. Brandt et al. (2019) employed this catalogue to constrain the masses of directly imaged companions, including the most precise mass estimate for Gl 758B. Kervella et al. (2019) presented an independent catalogue of Hipparcos-Gaia DR2 proper motion discrepancies, and use their results to confirm that Proxima Centauri is bound to $\alpha$ Cen, and set upper limits of $0.1-0.3 M_{\mathrm{J}}$ on the mass of potential planets orbiting Proxima within 1 to $10 \mathrm{au}$. They stress the power of Gaia and the Hipparcos-Gaia combination for the detection of long orbital periods, where the detection of tangential motion anomalies at accuracies of $1 \mathrm{~m} \mathrm{~s}^{-1} \mathrm{pc}^{-1}$ is already possible with Hipparcos and Gaia DR2. Future Gaia data releases will increase the sensitivity due to the increased time base line and the increased precision of the Gaia proper motions.

Finally, for the study of the early stages of planetary systems formation, the parallaxes provided by Gaia DR2 allow for distances to proto-planetary disk systems to be established to better then one percent precision. Hence a precise linear scale can be added to the spectacular images of disk substructure presented for example by Andrews et al. (2018).

4.1.3. Observational Hertzsprung-Russell diagrams. To illustrate the quality of Gaia DR2, Gaia Collaboration et al. (2018a) presented a set of splendid observational HertzsprungRussell diagrams. These reveal a wealth of detailed substructure, such as the binary sequence, a detailed view of the giant branch (Figs. 8 and 10 in Gaia Collaboration et al. 2018a), and the newly discovered split of the main sequence for halo stars into a blue and 

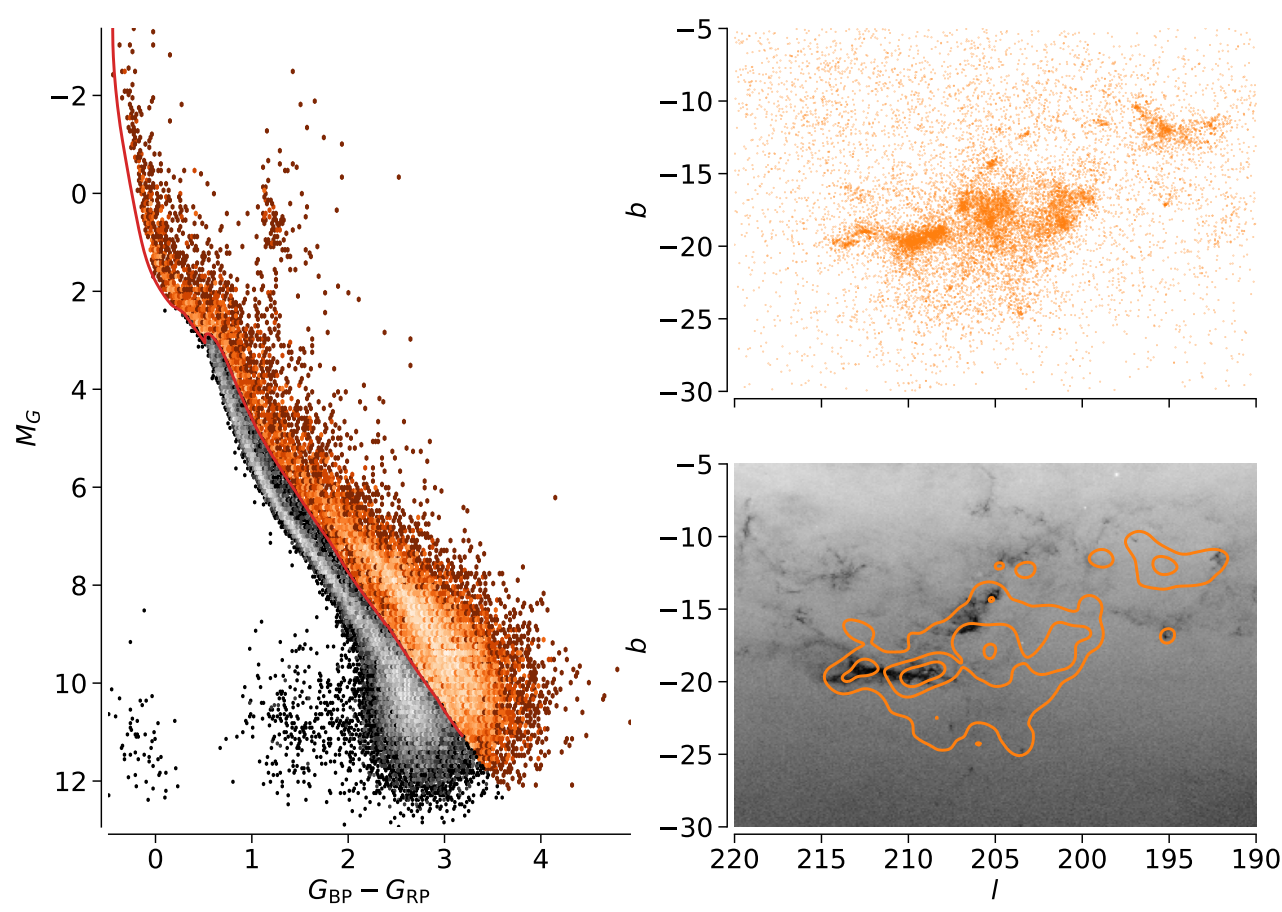

Figure 5

Selection of young stars in the Gaia DR2 observational HR diagram for the Orion region. The left panel shows the HR diagram for the 40167 stars selected as explained in the text. The line is a $14 \mathrm{Myr}\left(\log _{10}(\right.$ age $\left.)=7.15\right)$ solar metallicity isochrone from the MIST library (version 1.2, Choi et al. 2016 . Dotter 2016) and is used to separate the young population above the isochrone from the rest of the stars in the diagram. The top right panel shows the distribution of the young population (16692 stars) which is clearly very clustered and is closely associated with the dust features in the Orion region, as shown in the bottom right panel. The dust features are outlined as dark features in the map of number counts of stars located beyond $500 \mathrm{pc}$.

a red sequence (Figs. 20 and 21 in Gaia Collaboration et al. 2018a). This is a finger print of the formation history of the halo of the Milky Way which is discussed extensively in the review by Helmi (2020). The features in the HR-diagrams are visible thanks to the high precision parallaxes and photometry combined with the dense sampling of absolutemagnitude-colour space. Jao \& Feiden (2020) present a detailed analysis of the distribution of stars along the main sequence at $\left(G_{\mathrm{BP}}-G_{\mathrm{RP}}\right)>1.7$ and unveil many subtle features in the form of local density enhancements or gaps. The gap near $M_{G} \approx 10$, discovered by Jao et al. (2018), is confirmed and attributed to variability in stellar radii and luminosities over a narrow range in mass, $\sim 0.34-0.36 M_{\odot}$, caused by non-equilibrium ${ }^{3} \mathrm{He}$ fusion in the stellar core and intermittent mixing between core and envelope convection zones. The other features remain as yet unexplained but Jao \& Feiden (2020) speculate that subtleties in stellar atmospheric physics could be the cause. This is reminiscent of the features in the white dwarf observational HR diagram (Figure 6) discussed in the next section.

Gaia DR2 provides precise parallaxes for stars in the solar neighbourhood down to faint magnitudes. This allows for a new way to pick out the young stellar population by selecting 
low mass stars still located on the pre-main sequence, which are now easily recognizable by their position in the observational HR diagram, well above the main sequence in luminosity. A first application of this technique was presented by Villa Vélez et al. (2018) who showed for the Scorpio-Centaurus-Lupus sky region how a simple selection on parallax combined with isolating the young stars in the Gaia DR2 observational HR diagram beautifully reveals the sub-groups of the Sco OB2 association. Kounkel et al. (2018) applied the HR diagram selection to a combined Gaia DR2-APOGEE-2 sample to characterize the subgroups in the Orion star forming region. An illustration of the observational HR diagram selection of young stars, based solely on Gaia DR2, is shown in Figure 5 for the Orion region. The stars were selected from Gaia DR2 according to: $190^{\circ} \leq \ell \leq 220^{\circ},-30^{\circ} \leq b \leq-5^{\circ}$, $2.0 \leq \varpi \leq 3.5$ mas, $\varpi / \sigma_{\varpi} \geq 5$, and a proper motion filter selecting all stars within 5 mas $\mathrm{yr}^{-1}$ from $\left(\mu_{\alpha *}, \mu_{\delta}\right)=(-0.5,1)$ mas $\mathrm{yr}^{-1}$. In addition data quality filters were applied, requiring the renormalized unit weight error (RUWE, Lindegren 2018 to be less then 1.4 and applying Eq. C.2 from Lindegren et al. (2018). This results in a list of 40167 stars from which the young population can be selected as illustrated in the left panel of Figure 5. The rough selection of stars above the 14 Myr isochrone obviously includes a few evolved stars, but this does not affect the clearly clustered nature of the distribution of the young stars on the sky (top left panel). The bottom right panel of Figure 5 shows that, as one would expect, the selected young population is closely associated with the dust features in Orion. Zari et al. (2018) applied this combination of a simple kinematic and HR diagram selection to the full Gaia DR2 sample within 500 pc from the Sun and presented 3D maps of the spatial and age distribution of low mass pre-main sequence stars, as well as the spatial distribution of the population of young blue (OBA) stars. The maps outline the well known star forming regions around the sun, and according to Zari et al. (2018) cast doubt on the existence of the Gould Belt as an entity. They argue that the impression of a ring-like distribution of young stars is created by chance features in the 3D distribution (a conclusion reached earlier by Bouy \& Alves 2015, on the basis of Hipparcos data).

4.1.4. White dwarfs. Gaia DR2 for the first time allowed for the selection of field white dwarfs (WDs) directly from their location in the observational HR diagram, which previously was only possible for WDs in clusters (Gentile Fusillo et al. 2019). This allows for the construction of large all-sky samples of WDs, mostly free from colour and proper motion selection biases. Gentile Fusillo et al. (2019) presented a catalogue of almost half a million WD candidates, where the selection was guided by a spectroscopically confirmed sample from the Sloan Digital Sky Survey (see Gentile Fusillo et al. 2015, Hollands et al. 2017). The sample contains some 260000 high probability WD candidates. Jiménez-Esteban et al. (2018) combined Gaia DR2 and Virtual Observatory tools to select a sample of 73221 WDs, showing that the Gaia WD inventory is almost complete within 100 pc. Pelisoli \& Vos (2019) presented a catalogue of candidate extremely low-mass WDs.

A first exploration of the WDs in Gaia DR2 was presented in Gaia Collaboration et al. (2018a). The observational HR diagram for WDs with parallaxes better than $5 \%$ was examined (Figure 6), which showed unprecedented substructure, including a bifurcation of the WD sequence (features labeled "A" and "B" in Figure 6), as well as a branch "Q" running more or less horizontally across. Sequence A coincides with the cooling sequence for $0.6 M_{\odot}$ pure H atmosphere WDs (Gaia Collaboration et al. 2018a, Fig. 14). Sequence B shows the

\footnotetext{
8 https://www.cosmos.esa.int/web/gaia/dr2-known-issues\#AstrometryConsiderations
} 


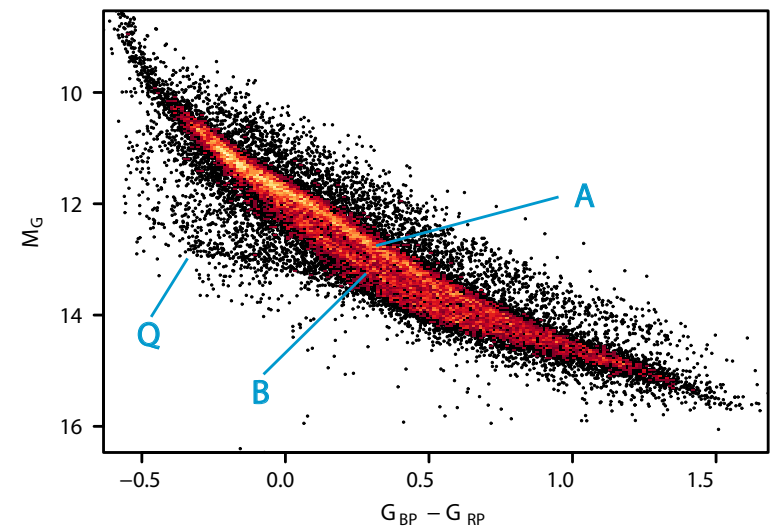

Figure 6

The Gaia DR2 observational Hertzsprung-Russell diagram for 26264 white dwarfs with parallaxes better than $5 \%$. The indicated branches of the white dwarf sequence are discussed in the text. Credits: Figure adapted with permission from Gaia Collaboration et al. (2018a); (C) ESO.

maximum separation from A around $\left(G_{\mathrm{BP}}-G_{\mathrm{RP}}\right)=0.1$ and at that point coincides with the $0.8 M_{\odot}$ pure H WD cooling sequence, which was unexpected. In colour-colour space the bifurcation of the WD sequence is also seen but in that case seems to correspond to a split between $0.6 M_{\odot}$ pure $\mathrm{H}$ and pure He atmospheres (the same puzzling result is shown in Jiménez-Esteban et al. 2018). With neither mass nor atmosphere effects seemingly able to explain the A and B sequences, several other explanations were put forward soon after the appearance of Gaia DR2. Kilic et al. (2018) proposed that a combination of both effects is needed and attribute the B sequence to relatively massive WDs that formed through mergers. El-Badry et al. (2018) showed that the bifurcation is still present in a sample of WDs all confirmed spectroscopically to be of the same atmospheric composition (DA) and propose that the shape of the field star initial-final mass relation is the cause of the bifurcation, with the slope of this relation being flatter for $3.5 \lesssim m_{\text {ini }} / M_{\odot} \lesssim 5.5$. The slope changes in the initial-final mass relation would then produce a secondary peak in the WD mass distribution at $\sim 0.8 M_{\odot}$, leading to sequence B. They explain the lack of bifurcations in observed cluster WD sequences by the need for a mixture of ages to produce the secondary mass peak. Bergeron et al. (2019) in an extensive study further investigated whether a higher than average WD mass or a different atmospheric composition is responsible for sequence B. They showed that the interpretation of the location of WDs in the HR or colour-colour diagram in terms of their mass is quite sensitive to the assumed atmospheric composition (an issue pointed out also in Gentile Fusillo et al. 2019). In particular the masses in sequence B would shift from 0.8 to $0.6 M_{\odot}$ when assuming a He atmosphere with a small admixture of $\mathrm{H}$ instead of a pure $\mathrm{H}$ atmosphere, which applies over the temperature range $\sim 12000$ to $\sim 5000 \mathrm{~K}$ where the split between sequences $\mathrm{A}$ and $\mathrm{B}$ is clearly visible. This leads to a picture of the Gaia DR2 observational WD HR diagram being populated mostly by "normal" white dwarfs of $\sim 0.6 M_{\odot}$, but with a varying $\mathrm{H} / \mathrm{He}$ mixture in the atmosphere as a function of temperature. At both high $(>12000 \mathrm{~K})$ and low temperatures $(<5000 \mathrm{~K})$ the different atmosphere compositions are then indistinguishable in colour-magnitude or colour-colour space. 
The detailed structure revealed in the Gaia DR2 observational HR diagram for WDs also constrains the interior structure of these stars. Tremblay et al. (2019) showed that the Q branch in the WD HR diagram (Figure 6) corresponds to white dwarfs that are undergoing crystallization of their interiors. The corresponding release of latent heat leads to a delay of the cooling, which takes WDs from the bright blue end of the HR diagram to the faint red end, by about a billion years. This causes a pile-up of WDs along the Q branch. This is the first observational confirmation of a prediction made over 50 years ago by van Horn (1968). Bergeron et al. (2019) showed in addition that the location of the crystallization sequence is quite sensitive to the interior composition of WDs, and showed empirically for the first time that, as expected, WD interiors consist of a mixture of carbon and oxygen. By analyzing the WD luminosity function, Tremblay et al. (2019) find strong evidence that in addition to the latent heat from crystallization WD cooling is further delayed by the energy released from ${ }^{16} \mathrm{O}$ sedimentation in the crystallizing cores. Cheng et al. (2019) focused their analysis on the high mass end of the Q branch and concluded that a small fraction of the $\sim 1.1 M_{\odot}$ WDs experience an additional cooling delay of 6-8 Gyr, which could possibly be explained by ${ }^{22} \mathrm{Ne}$ sedimentation in their interiors. Bauer et al. (2020) propose that the formation of solid clusters of ${ }^{22} \mathrm{Ne}$ in the liquid $\mathrm{C} / \mathrm{O}$ plasma enables the much more rapid sedimentation required to explain the long additional delay.

4.1.5. Open clusters. The field of open cluster studies received a tremendous boost from Gaia DR2, with the parallaxes, proper motions, and homogeneous all-sky two-colour photometry enabling the accurate characterization of known clusters as well as the discovery of many new ones. Many works focus on individual clusters and these will mostly not be discussed here. This overview is focused on the large-scale cluster studies that have been conducted with Gaia DR2 data.

The massive Gaia DR2 data set invites the application of machine learning methods and automated statistical analyses to the discovery and characterization of the many thousands of open clusters contained in the data. Cantat-Gaudin et al. (2018a) applied the unsupervised UPMASK method (Krone-Martins \& Moitinho 2014, Cantat-Gaudin et al. 2018b) to a list of known and claimed clusters and were able to determine membership and parameters for 1229 clusters. In addition 60 new clusters were discovered serendipitously in the fields examined. Castro-Ginard et al. (2018) developed a cluster identification method which uses DBSCAN (Ester et al. 1996) to find initial clusterings of sources in proper motion and parallax space, and then confirmed whether a cluster is found using an artificial neural network. Having found 23 new clusters, Castro-Ginard et al. (2019) then adapted this method for use on a supercomputer and applied it to the full Gaia DR2 subset at $|b| \leq 20^{\circ}$ and $G \leq 17$. They discovered 582 new open clusters in the Galactic disk, after accounting for overlaps with the Gaia DR2 cluster lists of Cantat-Gaudin et al. (2018a, 2019), Castro-Ginard et al. (2019), Sim et al. (2019), and Liu \& Pang (2019). Despite the bonanza of new clusters, the results from the search for open clusters in the Perseus and Galactic anti-centre directions (Cantat-Gaudin et al. 2019, Castro-Ginard et al. 2019) show that there is still a significant incompleteness in the inventory of open clusters within $\sim 2 \mathrm{kpc}$ from the sun. A related issue is that many of the previously catalogued open clusters turn out to be spurious, as shown in the works by Kos et al. (2018) and Cantat-Gaudin et al. (2018a). Kos et al. (2018) demonstrated that four sparse stellar aggregates with NGC numbers, classified as open clusters in 1888, are in fact chance projections on the sky. Most of the clusters not confirmed by Cantat-Gaudin et al. (2018a) are located at low Galactic latitudes and toward 
the inner disk. Cantat-Gaudin \& Anders (2020) made a particularly extensive effort to confirm open clusters claimed to exist in various literature sources. Their Fig. 3 shows that most of the clusters claimed to exist at large heights above the Galactic plane are spurious. This is in particular true toward the inner Galaxy, with NGC 6791 the only confirmed cluster above $500 \mathrm{pc}$ inside the solar circle. The work of extending and cleaning up the Milky Way open cluster inventory should continue as an accurate cluster inventory is essential, for example in understanding cluster disruption timescales as a function of their properties and the Galactic environment. Toward the fainter end of the Gaia survey $(G>18)$ the combination with complementary data from photometric and spectroscopic surveys will be crucial.

While the Gaia data readily allow for estimates of the distances and motions of open clusters, their characterization in terms of age and chemical composition, essential when using clusters to probe the evolution of the Milky Way, requires more painstaking efforts. In view of the large number of clusters with homogeneous data from Gaia DR2 automated methods for cluster characterization were thus developed and applied. Bossini et al. (2019) applied a Bayesian cluster parameter estimation method to a sample of 269 open clusters from Cantat-Gaudin et al. (2018a), focusing on low-extinction objects. They used only Gaia DR2 data which ensures a homogeneous determination of age, extinction and distance modulus for these clusters. The resulting ages show large differences with existing cluster catalogues, which may be due to the more reliable membership lists from Gaia combined with the precise and homogeneous photometry. Cantat-Gaudin et al. (2020) extended the existing Gaia DR2 open cluster inventory to a total of 2017 clusters, containing $\sim 230000$ stars brighter than $G=18$. They subsequently developed a neural network method for cluster parameter estimation (distance, age, reddening), which was trained mostly on the parameters from Bossini et al. (2019). Reliable parameters were obtained for 1867 clusters. The resulting cluster 3D positions were used to trace the structure of the Milky Way disk out to $\sim 4 \mathrm{kpc}$ (Figure 7). The young clusters (age $\lesssim 150 \mathrm{Myr}$ ) roughly trace the expected spiral arm pattern, outlining particularly well the Perseus and local arms. An apparent gap is seen in the Perseus arm, which Cantat-Gaudin et al. (2020) argue is physical. The reach of this Gaia DR2 open cluster sample is not quite enough to trace the outer disk or constrain the geometry of the warp. Anders et al. (2020) examined the distribution of ages as determined by Cantat-Gaudin et al. (2020) for 834 open clusters withing 2 kpc. Accounting for the completeness of the cluster sample as a function of age, they find that the age distribution is significantly shifted toward younger ages, peaking around $150 \mathrm{Myr}$, as compared to recent determinations based on the MSWC catalogue (Kharchenko et al. 2013, Piskunov et al. 2018, Krumholz et al. 2019). The open cluster age function as determined from Gaia DR2 thus seems compatible with the tidal disruption model proposed by Lamers et al. (2005) with a typical destruction timescale of $1.5 \mathrm{Gyr}$ for $10^{4} M_{\odot}$ clusters. Anders et al. (2020) estimate a current cluster formation rate of $0.55_{-0.15}^{+0.19} \mathrm{Myr}^{-1} \mathrm{kpc}^{-2}$, which for the typical cluster mass in their sample would imply that only $16_{-8}^{+11} \%$ of stars in the solar vicinity form in bound clusters.

For individual evolved open clusters the exquisite astrometry enables a much better characterization of the expected tidal tails that should be present around them. Two independent studies of the Hyades cluster by Meingast \& Alves (2019) and Röser et al. (2019) reveal the tidal tails extending over about $200 \mathrm{pc}$ in space, populated by hundreds of Hyades stars identified in the tidal tails (including 5 white dwarfs, Röser et al. 2019). In both studies the tidal tail structure clearly shows an S-shape when projected onto the 

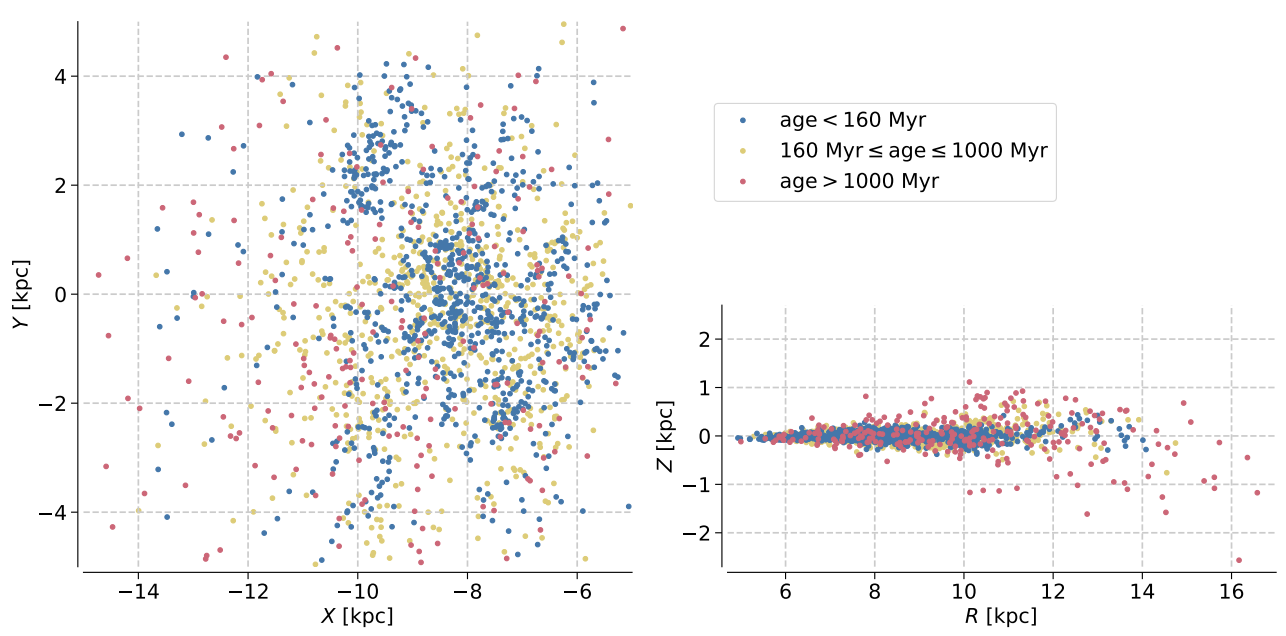

Figure 7

Projections of the 3D positions of 1867 open clusters listed in Cantat-Gaudin et al. (2020) in Galactocentric coordinates. The dots indicate the open cluster positions, colour coded by age as indicated in the legend. The left panel shows the locations of the 1799 clusters closest to the Sun projected onto the Galactic plane, with the Sun at $(-8.178,0)$. The young clusters along $X \sim-10$ roughly outline the Perseus spiral arm with the gap identified in Cantat-Gaudin et al. (2020) located at $(-10,1)$. The right panel shows the projected cluster positions in distance from the Galactic centre $R$ and vertical height $Z$. The most distant clusters Berkeley 29 and Saurer $1^{*}$ (at $(R, Z) \sim(20.5,1.8))$ are not included in the right panel. Credits: Open cluster data from Cantat-Gaudin et al. (2020).

Galactic plane, which corresponds well to predictions from $N$-body models of open clusters orbiting in the Gravitational potential of the Milky Way disk. Both Fürnkranz et al. (2019) and Tang et al. (2019) mapped the tidal tails around the Coma Berenices cluster and in the process discovered a neighbouring moving group separated by only a few $\mathrm{km} \mathrm{s}^{-1}$ in velocity space but without a cluster core. Meingast et al. (2019) discovered a large stellar stream close to the sun, also without a discernible core, and likely the remnant of a tidally disrupted cluster or association. The age of this system was estimated to be around 1 Gyr but this was revised downwards to $\sim 120 \mathrm{Myr}$ by Curtis et al. (2019). The younger age was confirmed in a follow-up study by Ratzenböck et al. (2020). Part of the confusion can be traced to the fact that searches done for (new) nearby stellar systems exclusively in Gaia DR2 are vulnerable to missing the high mass end, as those stars tend to be bright and may be missing from Gaia DR2 (Gaia Collaboration et al. 2018b). It thus remains important to complement Gaia DR2 data with Hipparcos data at the bright end.

The above studies hint at a large network of criss-crossing, relatively young (few hundred Myr), stellar clusters, associations, and streams in the solar neighbourhood. This network was mapped with Gaia DR1 data by Oh et al. (2017), while Kounkel \& Covey (2019) presented what appears to be complex filamentary structure in the young star distribution in the solar neighbourhood, which they proposed is primordial. Meingast et al. (2020) cast doubt on this finding on account of the very large velocity dispersion in the proposed filaments, and present robust evidence for an overlapping network of extended young clusters. Meingast et al. (2020) tentatively attribute this to a combination of primordial 
filamentary structure and tidal disruption. The picture is likely to grow more complex as the Gaia astrometry improves. Disentangling all the information into a coherent picture of the formation and disruption of stellar aggregates and their diffusion into the field star population cannot be done with spatio-kinematic studies only. Complementary photometric and spectroscopic data are required in order establish which stellar aggregates are co-natal, through age and chemical composition information. The interpretation must be guided by advanced simulations of star clusters dissolving into the Milk Way disk, such as those presented by Kamdar et al. (2019).

4.1.6. The phase spiral. Gaia Collaboration et al. (2018d) analyzed a sample of 6.4 million F-G-K stars, for which precise parallaxes $\left(\sigma_{\varpi} / \varpi<20 \%\right)$ and full 6 D phase space information are available from Gaia DR2, and mapped the Milky Way disk kinematics between 5 and $13 \mathrm{kpc}$ from the Galactic centre and up to $2 \mathrm{kpc}$ above and below the disk midplane. The results show a very rich and complex velocity field in the Galactic disk, strongly confirming the growing evidence from the past decade that the Milky Way disk is not in equilibrium. The most spectacular evidence for a disturbed disk was presented by Antoja et al. (2018) in the form of the "phase spiral" visible in the distribution of stars in the plane of vertical velocity $v_{z}$ vs. vertical position $z$ with respect to the Galactic plane. Figure 8 shows the phase spiral which is visible directly in the number counts in the panel on the left, but is even more clearly visible in the panel on the right when the bins in $z-v_{z}$ are colour coded by the median azimuthal velocity $v_{\phi}$ of the corresponding stars. The sample used for this figure consist of 764370 stars selected to have precise parallaxes, good quality astrometry (renormalized unit weight error less than 1.4), reliable radial velocities (removing the potentially spurious radial velocities listed in Boubert et al. 2019), and located in galactocentric cylindrical radius at $\left|R-R_{\odot}\right| \leq 100$ pc. The barycentric Cartesian velocities were transformed to galactocentric cylindrical coordinates as described in Antoja et al. (2018), using different values for $R_{\odot}\left(8178 \mathrm{pc}\right.$, Gravity Collaboration et al. 2019) and $z_{\odot}$ (20.8 pc, Bennett \& Bovy 2019). By combining Gaia DR2 with spectroscopic data from the second data release of the GALAH survey (Buder et al. 2018), Bland-Hawthorn et al. (2019) showed that the phase spiral is visible at different galactocentric radii and azimuths and across different stellar populations (age, metallicity), thus demonstrating that Antoja et al. (2018) discovered a disk-wide phenomenon.

Antoja et al. (2018) pointed out the similarity of the phase spiral to the process of phase mixing in two dimensional systems (cf. Fig. 1 in Tremaine 1999), implying that the disk of the Milky Way was disturbed relatively recently on a cosmological timescale. They presented a toy model of a disturbed self-gravitating disk which reproduced qualitatively the phase spiral. Binney \& Schönrich (2018) and Darling \& Widrow (2019) presented more sophisticated models, showing how the effects of a massive intruder passing through the Galactic plane or bending waves excited in the disk manifest as spirals in the $z-v_{z}$ plane. A massive intruder would in fact excite waves in the disk (e.g., Laporte et al. 2018) and Antoja et al. (2018) pointed to the previous pericentric passage of the Sagittarius dwarf galaxy as the event that caused the observed phase spiral. Detailed numerical simulations presented in Bland-Hawthorn et al. (2019) strengthened the case for this explanation, with the excitation of the spiral timed at $\sim 0.5$ Gyr ago. Although Laporte et al. (2019) showed that the phase spiral was visible in simulations that were run prior to the appearance of Gaia DR2 (Laporte et al. 2018), none of the pre-Gaia DR2 works on disturbances of the Milky Way disk predicted the specific features unveiled by Antoja et al. (2018). 

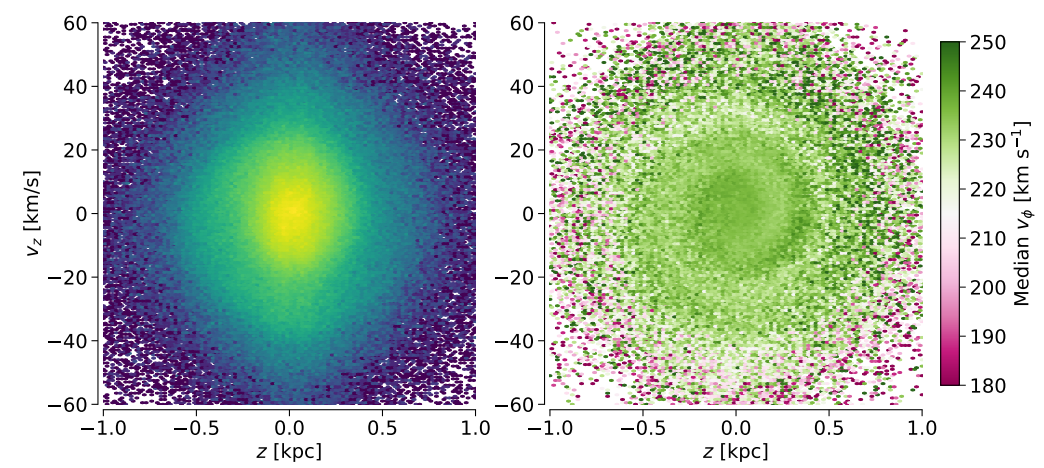

Figure 8

The distribution of stars near the solar circle in the vertical position-velocity plane. The data are shown for stars selected on their cylindrical Galactic coordinate according to $\left|R-R_{\odot}\right| \leq 100 \mathrm{pc}$. The colour coding in the left panel represents the star number counts in bins of $\Delta z=0.02 \mathrm{kpc}$ by $\Delta v_{z}=1 \mathrm{~km} \mathrm{~s}^{-1}$. In the right panel the colour coding shows the median values of $v_{\phi}$ for the same bins. Credits: Figure adapted by permission from Springer Nature: Nature 561:360 (A dynamically young and perturbed Milky Way disk, T. Antoja et al., left and right panels of their Fig. 1) (C) 2018.

Further observational evidence that the Sagittarius dwarf is related to the phase spiral was uncovered by Ruiz-Lara et al. (2020) through a stellar population synthesis analysis of the Gaia DR2 observational HR diagram of stars within $2 \mathrm{kpc}$ from the sun. The star formation history they inferred shows peaks at 5.7, 1.9, and $1 \mathrm{Gyr}$ ago, coinciding with the past pericentre passages of the Sagittarius dwarf galaxy. This suggests that the Sagittarius dwarf may have played an important role in the build up of the stellar mass of the Milky Way's disk, where it is a curious coincidence that the somewhat extended peak in the star formation history around 5.7 Gyr ago covers the interval in time some 4.6 Gyr ago during which the Sun and the solar system formed.

Bland-Hawthorn \& Tepper-Garcia (2020) elaborate the model of Binney \& Schönrich (2018) to further understand how the phase spiral is excited and how it evolves after an impulsive disturbance. They time the emergence of the phase spiral at about $380 \mathrm{Myr}$ after the last passage of the Sagittarius dwarf through the Galactic disk. Fig. 7 in BlandHawthorn \& Tepper-Garcia (2020) shows how the impact induces an $m=2$ bending mode in the disk. The vertical displacement evolves in time and azimuth around the disk which is compatible with the conclusion reached by Poggio et al. (2020) that the warp in the disk is precessing, likely as the consequence of a recent or ongoing encounter with a satellite galaxy. It seems clear that the Sagittarius dwarf galaxy and the phase spiral are linked but there is work to be done to tie the whole story together neatly. The observation of the phase spiral together with advances in the mapping of the Sagittarius stream (Antoja et al. 2020, Ramos et al. 2020) illustrates that, as implied by Bland-Hawthorn \& Tepper-Garcia (2020), with Gaia DR2 the era of "Galactic seismology" has started (the term Galactoseismology was introduced by Widrow et al. 2012).

4.1.7. Stellar streams. Prior to the launch of Gaia, Ivezić et al. (2012) reviewed the impact of the large photometric and spectroscopic surveys that were in operation during the first decade of the 21st century. These surveys clearly established the complex and dynamical 
nature of the Milky Way. In particular the Sloan Digital Sky Survey opened up the possibility of peering deep into the halo and through matched filter techniques pick out many substructures, including streams 9 , thought to be remnants of dwarf galaxies or globular clusters disrupted by tidal forces as they orbit in the gravitational potential of the Milky Way. The release of Gaia DR2 clears our view of streams in the halo by filtering out the foreground stars on the basis of their large parallaxes and/or proper motions, and at the

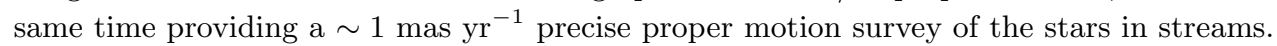

Gaia DR2 led to a substantial expansion of the inventory of streams criss-crossing the halo, in particular thanks to the application of the STREAMFINDER algorithm (Malhan \& Ibata 2018) to the Gaia DR2 astrometric and photometric data for sources beyond $1 \mathrm{kpc}$ and at high latitude $\left(|b|>20^{\circ}\right.$, Malhan et al. 2018, Ibata et al. 2019b). Many known streams were identified as well as candidate streams which may be artifacts of the Gaia scanning law and await confirmation. Five new streams were identified beyond $5 \mathrm{kpc}$ and eight new structures in the inner halo at heliocentric distances between 1 and $10 \mathrm{kpc}$. Of the latter the "Fimbulthul" stream is noteworthy as it was subsequently identified as the tidal stream of the $\omega$ Centauri globular cluster (Ibata et al. 2019a), lending strong support to the suspicion that $\omega$ Cen is the remnant core of an accreted dwarf galaxy.

Many studies using Gaia DR2 concentrated on individual streams, the results for two streams are highlighted here. The study of the GD-1 stream by Price-Whelan \& Bonaca (2018) is a beautiful illustration of the power of Gaia at filtering out foreground stars. By considering only stars at $\varpi<1$ mas in the sky region around GD-1, Price-Whelan \& Bonaca (2018) were able to narrow down the GD-1 candidates through a proper motion selection, and then applied a matched filter to Pan-STARRS photometry to isolate the stars in Gaia DR2 that are members of the stream. Gaia DR2 transformed the view of GD-1. The mapping of GD-1 was extended by $20^{\circ}$ across the sky such that it now extends over $80^{\circ}$. In addition plausible locations for the stream progenitor and gaps within the stream were identified, as well as stars that are clearly part of the stream but located off the main stream track. Modelling by Bonaca et al. (2019), aimed at explaining the stream gaps as well as the off-track stars, suggested that GD-1 may have been perturbed by a massive object, the models preferring a dense perturber with a mass in the range $10^{5}-10^{8} M_{\odot}$. No known globular cluster could be identified as the possible perturber, leaving as a tantalizing possibility that the perturber was a dark matter subhalo, predicted to exist in $\Lambda$ CDM. In a follow-up study Bonaca et al. (2020) acquired high resolution spectroscopy for the off-track stars and confirmed that they are kinematically and chemically consistent with the main stream. The radial velocity offset between the stream and the spur then allows us to constrain the orbit of the perturber. The possible perturber orbits overlap with the present day debris of the Sagittarius stream, suggesting that the perturber may have originated from the Sagittarius dwarf galaxy. An interaction with the Sagittarius dwarf was also suggested by de Boer et al. (2020), who further extended the mapping of GD-1. Ibata et al. (2020) re-examined the GD-1 stream, adding radial velocity information and investigating sample contamination, and concluded that the apparent gaps in the stream can also be explained as the projected morphology caused by epicyclic motion of the stream stars in a smooth Galactic potential. In addition Ibata et al. (2020) point out that the sky region around GD-1 shows artifacts in the Gaia completeness function (see their Fig. 10)

\footnotetext{
${ }^{9}$ The stellar streams discussed here concern those recognizable as such in configuration space. Streams identified as overdensities in the space of integrals of motion are not considered.
} 
that are clearly related to the scanning law and could cause or enhance the appearance of gaps. The picture of GD-1 thus appears quite complex. Further insights will come from a better understanding of Gaia selection function effects, a cleaner GD-1 sample, and more complete modelling in which GD-1 is orbiting in the halo in the presence of larger systems such as the Sagittarius dwarf galaxy.

The advantages of an all sky astrometric and photometric survey are very well illustrated by the study of the Orphan stream by Koposov et al. (2019). No longer constrained by the footprint of surveys such as SDSS, they used and all-sky RR Lyrae sample extracted from Gaia DR2 to guide the subsequent selection of members of the Orphan stream. The stream now spans $\sim 210$ degrees across the sky, translating to some $150 \mathrm{kpc}$ in physical length. This led to the detection of the changing orbital pole position along the stream, which corresponds to twists in its shape. In addition the proper motions show a strong acrosstrack component. These observations point the Orphan stream having been perturbed by a massive object. Modelling by Erkal et al. (2019) showed that a perturbation of the Orphan stream by the Large Magellanic Cloud very nicely explains the observational data. The model for the Orphan stream in the combined potential of the Milky Way and the LMC can be used to estimate the mass of the latter as $1.38_{-0.24}^{+0.27} \times 10^{11} M_{\odot}$. In addition the Milky Way mass within $50 \mathrm{kpc}$ is estimated to be $3.80_{-0.11}^{+0.14} \times 10^{11} M_{\odot}$ and the prediction is made that the reflex motion of the Milky Way in response to the LMC should produce a bulk upwards motion of the stellar halo. These two studies of the Orphan stream demonstrate the need for a much more complex modelling of streams orbiting in the Milky Way potential. The effects of massive perturbers such as the LMC on both the streams and the Milky Way must be accounted for in order to properly interpret the observations.

4.1.8. From globular clusters to the Andromeda nebula. The studies of tidal streams above demonstrate the capability of Gaia to probe the Milky Way far out into the halo. Indeed many globular clusters, dwarf galaxies, and even the M31 and M33 galaxies are within reach of Gaia astrometry. A first demonstration of the reach of Gaia DR2 was provided by Gaia Collaboration et al. (2018c), who presented the proper motions and orbits of 75 globular clusters, nine dwarf galaxies, one ultra-faint system and the Small and Large Magellanic Clouds. Several works followed up on and extended the results presented.

The orbits of dwarf galaxies provide constraints on the Milky Way's mass. Gaia Collaboration et al. (2018c) assumed that the Leo I dwarf galaxy is bound and derived a Milky Way mass of $0.9 \times 10^{12} M_{\odot}$. Fritz et al. (2018) examined the proper motion data for 39 (candidate) dwarf galaxies out to $420 \mathrm{kpc}$ and find that the observed satellite kinematics are best explained by their high mass halo alternative of $1.6 \times 10^{12} M_{\odot}$. Callingham et al. (2019) used the EAGLE cosmological hydrodynamics simulations to predict the distribution function for satellite galaxies. They infer the Milky Way's halo mass by calculating the likelihood that the observed satellite population is drawn from this distribution function and find a value of $1.2 \times 10^{12} M_{\odot}$. Eadie \& Juric (2019) use the Gaia DR2 globular cluster kinematics to estimate the total mass of the Milky May and arrive at $0.7 \times 10^{12} M_{\odot}$. The latter study relies on the 150 globular cluster proper motions derived from Gaia DR2 by Vasiliev (2019), who derived the mean proper motions after accounting for systematic errors. The Milky Way mass derived by Vasiliev $(2019)$ is $1.2 \times 10^{12} M_{\odot}$ with large uncertainties. The division into low $\left(\sim 10^{12} M_{\odot}\right)$ and high $\left(\sim 1.6 \times 10^{12} M_{\odot}\right)$ estimates for the Milky Way mass (Bland-Hawthorn \& Gerhard 2016) is still present but will likely be resolved once Gaia EDR3 is available. 
Besides the orbits and the distribution function of globular clusters Gaia Collaboration et al. 2018c, Vasiliev 2019) these systems can also be studied in detail individually with Gaia. Gaia Collaboration et al. (2018c) demonstrated that the signature of rotation can clearly be detected with Gaia DR2 in at least five globular clusters. This was extended by Bianchini et al. (2018) to the detection of rotation at the $3 \sigma$ level for 11 globular clusters. For eight clusters it is in addition possible to constrain the inclination angle of the rotation axis with respect to the line of sight. The rotation of globular cluster can be measured thanks to the availability of proper motions for large number of stars in each cluster. In the Magellanic Clouds many millions of stars are observed by Gaia and consequently Gaia Collaboration et al. (2018c) were able to present exquisite kinematic maps for the LMC and SMC, deriving a proper motion based rotation curve for the LMC that is competitive with line-of-sight velocity curves. Vasiliev (2018) used half a million red giants in the LMC to derive a two-dimensional kinematic map, consisting of the mean proper motion and its dispersion out to $7 \mathrm{kpc}$ from the LMC centre. This kinematic map was used to measure the rotation curve, the velocity dispersion profile, and the orientation of the LMC. In a sense Gaia provides the tangential motion equivalent of the line-of-sight kinematic maps derived from integral field unit spectrograph data for distant galaxies.

Moving further out into the local group van der Marel et al. (2019) selected stars in M31 and M33 from Gaia DR2, taking care to remove contaminant sources and bad measurements. The resulting sample of stars allows the measurement of the mean proper motions of M31 and M33, but more impressively, an averaging of the proper motions over sectors in azimuth around the two galaxies reveals the signature of their rotation. These are consistent with the literature but at the moment are not very constraining. The rapid improvement in proper motion precision for future Gaia data releases (see Section 5 ) should permit rotation measurements that are competitive. The mean proper motions measured with Gaia DR2 were combined with existing measurements as inputs to numerical orbit integrations, the results of which imply that M33 must be on its first infall with respect to M31. The Gaia DR2 proper motion for M31 also implies that it is on a less radial orbit toward the Milky Way than found from HST proper motions. Hence a future collision with the Milky Way will happen somewhat later and at a larger pericentre. Tepper-García et al. (2020) used the Gaia DR2 proper motions measurements in a combined $N$-body and hydrodynamical simulation of the M31 and M33 orbital history that takes into account the effects of dynamical friction and of mass loss due to tidal stripping. They conclude that the Gaia DR2 proper motions do allow for a second-infall scenario for M33, with the first encounter 6.5 Gyr ago bringing these galaxies to within $\sim 50 \mathrm{kpc}$ of each other.

4.1.9. QSO and AGN studies. This overview of Gaia DR2 science results started in the solar system where Gaia observes any minor body that is bright enough and sufficiently pointlike in appearance to its telescopes. At the other end of the universe Gaia automatically observes galaxies that have bright cores as well as distant quasars which are point-like. Gaia in principle could observe tens of millions of galaxies located at redshifts up to $\sim 0.3$ (Robin et al. 2012), but many of these will not be selected for observation on board (de Bruijne et al. 2015). Of order 1 million galaxies are expected to be observed by Gaia and these can be morphologically characterized at the $0.2^{\prime \prime}$ level, which represents a unique all sky survey (Krone-Martins et al. 2013). The characterization of galaxies is part of the data products for future Gaia data releases.

Gaia DR2 contains 550000 identified quasars which materialize the celestial reference 
frame, but also form an interesting sample scientifically. Gaia Collaboration et al. (2018e) presented and examined the properties of the Gaia-CRF2 and it was noted that a small fraction of the ICRF3 sources in Gaia DR2 show significant offsets between their radio and optical positions, even after the alignment of the optical and radio frames. It is well-known that there can be physical reasons for such offsets, the optical centre of emission for a QSO or AGN does not necessarily coincide with the radio centre. This was exploited by Plavin et al. (2019) who analyzed the radio-optical position offsets for a sample of $\sim 4000$ AGN. For the subset with the most precisely determined radio jet position angles they found that the position offsets for $\sim 70 \%$ of the sources are along the radio jet. Due to synchrotron opacity effects, the core of the radio emission is expected to be slightly offset $(<1$ mas) from the AGN nucleus downstream along the jet, while the core of the optical emission will be determined by the relative brightness of the nuclear region and jet in the optical. The observed downstream offsets of the optical positions are explained by the presence of optical jets $20-50$ pc long. The upstream offsets are explained as the accretion disk dominating the optical source position, with possibly a minor contribution from radio offsets due to unaccounted for structure in the radio jet or frequency-dependent synchrotron opacity. The Plavin et al. (2019) sample is large enough to allow for studies of the radio-optical offsets as a function of redshift and colour and the results indicate that the distribution of offsets is consistent with the unified scheme for AGN classification.

The high spatial resolution of the Gaia sky map combined with its high completeness to $G \sim 20$ makes it a great tool to search for gravitational lens systems harboring multiply imaged quasars. Several groups capitalized on this, discovering new quasar lenses in imaging surveys such as DES and Pan-STARRS, guided by the Gaia DR1 catalogue (e.g., Agnello et al. 2018, Ostrovski et al. 2018). The improvements in the completeness and angular resolution in Gaia DR2 prompted automated searches for clusters of sources in the sky map that could be the images of a lensed quasar. Delchambre et al. (2019) conducted a blind search for lenses by extracting some 2 million source clusters from Gaia DR2, and then using simulations to train a machine learning model to identify promising lensed quasar candidates, of which 15 were confirmed as newly identified quadruply lensed quasars. Starting from a more targeted search, guided by existing quasar catalogues, Krone-Martins et al. (2018) presented two new quadruply imaged quasars which were entirely discovered in Gaia DR2. Ducourant et al. (2018) present a detailed analysis of the Gaia DR2 positions of the HE0435-1223 system and show that the precision of the Gaia astrometry allows for significantly better constraints on the parameters of the lensing model compared to HST astrometry, and that parameter correlations are significantly reduced. Future Gaia data releases will feature gravitational lens catalogues thus promising a bright future for studies of strong gravitational lensing.

4.1.10. Creative uses of the Gaia data. Massive and richly annotated data sets such as the Gaia data releases lend themselves to investigations that go beyond the traditional or obvious uses of the data. A few examples of creative uses of Gaia DR1 and DR2 are highlighted here. The potential of the Gaia DR1 sky map for the discovery of new stellar systems was realized by Koposov et al. (2017), who produced an all-sky map of potential source over-densities. This led to the discovery of two new star clusters, one of which, Gaia1 , had always been hiding in plain sight right next to Sirius. This discovery was possible thanks to anti-blooming measures in the Gaia CCDs (Crowley et al. 2016) which enable high spatial resolution to be maintained even very near the brightest stars in the sky. The 
reality of this cluster was confirmed in a spectroscopic follow-up study by Simpson et al. (2017) who concluded that Gaia-1 is a rather massive $\left(\sim 10^{4} M_{\odot}\right)$ cluster with a metallicity of $[\mathrm{Fe} / \mathrm{H}]=-0.13 \pm 0.13$ and an age of about 3 Gyr. The orbit of the cluster as calculated by Simpson et al. (2017) shows that it can travel as far as $\sim 1 \mathrm{kpc}$ from the Galactic plane, perhaps making this a thick disk cluster. Koch et al. (2018) measured an iron abundance of $-0.62 \pm 0.1$ and a moderately enhanced $\alpha$-element abundance, thus providing further evidence that Gaia-1 is part of the thick disk population. The precise nature of Gaia1 remains unclear. Mucciarelli et al. (2017) find a solar abundance for the cluster, and Carraro (2018) disputes the association of Gaia-1 with the thick disk by pointing out that the height of the cluster above the warped disk is less than $0.5 \mathrm{kpc}$.

To track down variables stars for which no light curves were published in Gaia DR1 (except for the modest sample presented in Clementini et al. 2016), Belokurov et al. (2017) used the fact that the photometric uncertainties quoted in Gaia DR1 reflect the scatter in the individual observations made for each source. This leads to overestimates of the uncertainty on the mean $G$ band value for variable sources, making these stars stand out in a diagram of the uncertainty in $G$ vs. $G$. By calibrating against samples of known variable stars Belokurov et al. (2017) were able to identify candidate RR Lyrae stars in a field covering the Magellanic clouds. These candidate RR Lyrae beautifully outline the LMC and SMC and in particular reveal the bridge of old stars between the two Milky Way companions. A combination of Gaia DR1 with GALEX data revealed the existence of a bridge of younger stars, offset from the bridge of old stars and coincident with the known HI bridge between the LMC and SMC.

In a similar vein, Belokurov et al. (2020) examined the astrometric data quality indicators in Gaia DR2 in order to identify potential unresolved binaries across the observational HR diagram for sources within $400 \mathrm{pc}$ from the sun. The sources they selected for study generally have good quality astrometric solutions as indicated by the value of the RUWE, which peaks around unity. However there is a tail of sources with RUWE values up to 4 and these could be unresolved binaries as pointed out in Lindegren (2018). Belokurov et al. (2020) showed that the positions in the HR diagram where binaries are expected (such as the location 0.75 magnitudes brighter than the main sequence) indeed manifest elevated median values of the RUWE. A translation between the RUWE value and the binary component separation on the sky was proposed and verified against known spectroscopic binaries. This allowed Belokurov et al. (2020) to apply their binary detection method to various science cases. In one application they show that for stars hosting high-mass Jupiters the peak in the RUWE distribution shifts away from unity for hosts where the planet is orbiting close to the star. This suggests that the existence of hot Jupiters can be explained as the consequence of inward migration driven by the effects of another companion of the host star, perhaps through the Kozai-Lidov mechanism. This finding from Gaia DR2 astrometric data quality indicators may be confirmed when in future Gaia data releases binary and exoplanet catalogues based on the epoch astrometry will appear.

Voggel et al. (2020) show how astrometric and photometric data quality indicators in Gaia DR2 can be used to search for globular clusters and tidally stripped galaxy nuclei around galaxies out to $\sim 25 \mathrm{Mpc}$. Applying this to the case of Centaurus A they were able to identify 632 candidate luminous globular clusters in the halo of this galaxy, of which 5 have been confirmed as bona fide clusters through follow-up spectroscopy. 


\subsection{Some lessons learned}

I list here a few lessons learned from the scientific exploitation of the Gaia DR1 and in particular Gaia DR2 catalogues which have implications for future directions in survey astronomy.

- The first Gaia data release already made clear that indeed this mission will deliver on the promise to revolutionize studies of the Milky Way and play a key role in unravelling the formation history of our home galaxy. This was evident from the study by Helmi et al. (2017), and indeed as the title of that paper states, the Gaia data releases represent a "box full of chocolates", containing a flavour for every astronomical taste as amply demonstrated by the breadth of topics discussed above. The main and lasting impact of Gaia therefore is the availability to the astronomical community of fundamental data for sources all across the sky, in the form of accurate astrometry, photometry, and radial velocities. This makes Gaia an anchor for many other surveys, either as an astrometric, photometric, or radial velocity reference or as a source for well characterized observing targets. As a consequence, Gaia DR2 has quickly become an indispensable part of the astronomical ecosystem.

- Many of the studies discussed above rely on the combination of Gaia DR2 and other photometric and/or spectroscopic surveys. The latter benefit from the addition of 3D spatial and kinematic data provided by Gaia, while the Gaia astrometry and radial velocities are really only useful in combination with the astrophysical characterization of sources. In designing future survey strategies from which to decide on instruments to build, this powerful combination of astrometry, photometry, and spectroscopy should be kept in mind, where for example priority could be given to the collection of the fundamental data most limiting to the astronomy applications at a given time.

- The discovery and subsequent follow-up work on the phase spiral (Section 4.1.6), as well as the Galactic archaeology work on the Galactic halo reviewed by Helmi (2020), demonstrate that dense sampling of Galactic phase space is essential to uncover subtle but important features in the phase space of our galaxy. The dense and precise sampling of phase space also fully enables the field of Galactic seismology. This argues for massive surveys, where in the disk it should be possible to penetrate the obscuring dust. The dense phase space sampling combined with precise parallaxes and photometry also allows for discovering and studying subtle features in the observational $\mathrm{HR}$ diagram (Sections 4.1.3 and 4.1.4.

- Although Gaia DR2 represents an intermediate Gaia data release, based on a fraction of the amount of data that will eventually be collected, its legacy value is already immense in that it would represent the standard for the coming decade in terms of the astrometric and photometric precision. Future releases superseding Gaia DR2 will have even longer lasting legacies and it should thus be ensured that the Gaia data archive remains technically up to date and easily interoperable with other surveys. There is also a very strong case for making available lower level data products from Gaia to enable a future reprocessing of (parts of) the data (Brown 2010, 2012).

- Conspicuously absent from the major results obtained with Gaia data is the determination of basic structural parameters of the Milky Way, such as the radial profile of disk stars (for the pre-Gaia review of Galactic structural parameters see BlandHawthorn \& Gerhard 2016). The main reason is the lack of a well described selection 
function for the Gaia data releases. A major task for the coming years will be to derive the selection function in order to ensure that the exquisite positional and kinematic data provided by Gaia can be used for population studies. The first very encouraging attempts have been made (Boubert et al. 2020, Boubert \& Everall 2020), demonstrating the need to engage the DPAC to ensure that as yet unpublished information relevant to the Gaia selection function is made public.

- A lesson very much taken to heart by the community is that the majority of the Gaia parallaxes will remain at relative accuracy levels which make it dangerous to directly invert their values in order to estimate distances (see the extensive discussion in Luri et al. 2018). Nevertheless, all the Gaia parallax information should be used to constrain or calibrate alternative distance indicators which can then extend the reach of Gaia, for instance to ensure that the full relative accuracy of proper motions can be exploited. Standard candles are a natural choice but ideally (photometric or spectroscopic) distance indicators across the HR diagram should calibrated and progress is being made in this direction. The use of parallaxes in combination with spectroscopy and/or photometry to make accurate Bayesian distance estimates was demonstrated by Sanders \& Das (2018) and Anders et al. (2019). The first steps toward accurate luminosity calibrations across the HR diagram on the basis of Gaia parallax information were taken by Leistedt \& Hogg (2017), Anderson et al. (2018), and Cranmer et al. (2019).

\section{PANORAMA FOR THE COMING DECADE}

\subsection{Upcoming Gaia data releases}

The astronomical community can look forward to more Gaia data releases over the coming decade. The next release, Gaia EDR3 will appear in December 2020 and provides updated astrometry and photometry based on 34 months of observations (Gaia Collaboration et al. 2020). The parallaxes will improve by $20 \%$ in precision but for the proper motions the improvement will be a factor of 2 , thanks to the longer time baseline of the observations (see below). The full Gaia DR3 will appear in 2022 and will contain: updated and new radial velocities (for some 30 million stars out to $G_{\text {RVS }} \sim 14$ ); astrophysical parameters for sources based on the BP/RP prism and RVS spectra; the prism and RVS spectra for a subset of sources; an extended catalogue of variable stars; the first catalogue of binary stars, including eclipsing, spectroscopic, and astrometric binaries; astrometry for some 100000 solar system objects and reflectance spectra for a subset of $\sim 5000$ asteroids; QSO host and galaxy morphological characterization; and the light curves for all sources in a $5.5^{\circ}$ radius field around M31. This illustrates that the science topics such as discussed in Section 4.1 will profit from an increasingly rich set of Gaia data providing the astrophysical characteristics of sources.

Gaia DR4 will be based on all data collected during the nominal mission lifetime and a part of the extended mission (5.5 years of observations), and will feature parallaxes that are more precise by a factor 1.7 with respect to Gaia DR2, while for proper motions the gain is a factor of 5.2. This release will feature an exoplanet catalogue, predicted to contain some 21000 Jupiter mass planets (Perryman et al. 2014). However the main change that Gaia DR4 will introduce is the release of all the epoch data from Gaia, meaning for each source the astrometric, photometric, radial velocity, and BP/RP/RVS spectra times series. This represents a vast volume of data and creates significant new possibilities, where for 


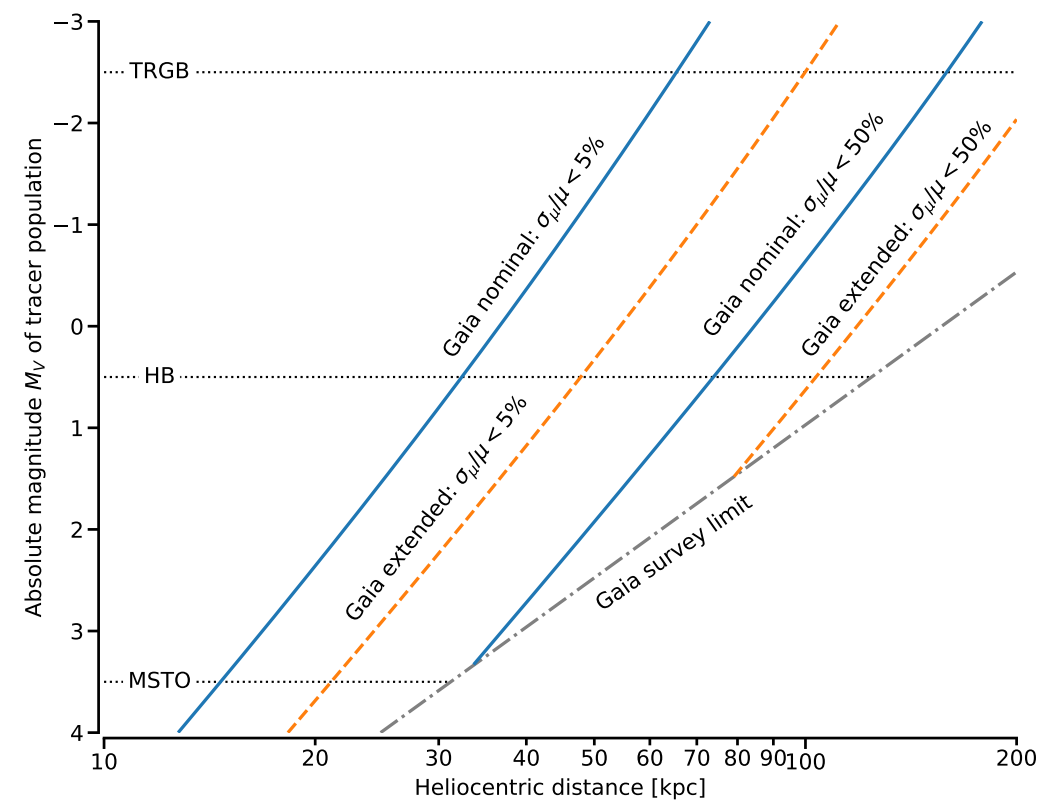

Figure 9

Predicted relative proper motion uncertainty $\sigma_{\mu} / \mu$ for three tracer populations in the Absolute magnitude vs. distance plane. The tracers are the main sequence turnoff (MSTO), horizontal branch (HB), and tip of the red giant branch (TRGB) stars. The lines show contours of constant $\sigma_{\mu} / \mu$ for the nominal 5-yr Gaia mission (solid lines) and the extended 10-yr mission (dashed lines). The dot-dashed line indicates the Gaia survey limit $(G=20.7)$. The diagram illustrates the gain in distance out to which a certain relative proper motion uncertainty can be obtained when doubling the Gaia mission lifetime. Note the logarithmic horizontal scale. Credits: Figure adapted with permission from Mateu et al. (2017), their Fig. 15.

example the community can conduct independent searches for companions to Gaia sources using the epoch astrometry.

On July 162019 the nominal five year Gaia mission ended and the project transitioned to the extended mission phase. It is expected that Gaia can continue to operate until early 2025, the limiting factor to its lifetime being the propellant for the micro-propulsion system (see Gaia Collaboration et al. 2016b, Sect. 3.2.1). Once the fine attitude control is lost the images collected by Gaia will no longer be of the quality needed for astrometry. The Gaia mission has formally been extended to the end of 2020, with further extension pending approval. It is expected however that a full ten year mission can be realized. This doubling of the mission lifetime means the precision for all Gaia data products, including parallaxes will improve by $40 \%$ (reflecting the $\sqrt{t}$ behaviour of the signal to noise). For proper motions the improvement also includes the longer time baseline, the precision improving proportional to $t^{1.5}$, which implies a factor of 2.8 better proper motions (with respect to the 5 year mission) at a given magnitude or an increase by a factor 22 of the volume over which a given proper motion precision can be achieved. This is visualized for a range of bright stellar tracer populations in Figure 9 For more complex motions in binaries and exoplanet systems the gains are even more impressive. Measuring perturbations of the assumed motion on the sky for a single star is equivalent to measuring second and higher order derivatives of the 
celestial positions with respect to time (the proper motion being the first derivative). Each derivative of time introduces another factor of $t$ improvement, hence acceleration sensitivity increases by a factor of 5.6. Unambiguously determining the orbital period, mass, and distance of the perturbing body is only possible when the fourth derivative of the position can be measured (shown by L. Lindegren in the proposal submitted to ESA for the extended Gaia mission). In this case the sensitivity increases by a factor of 22.6. Doubling the mission lifetime also means that the sensitivity to long orbital period companions increases, leading for example to an increase in exoplanet detections to $\sim 70000$ (Perryman et al. 2014). For solar system objects the 10 year mission lifetime would allow measuring astrometry for main belt asteroids over two orbital periods, and for Trojans a full orbit would be covered. There is thus much to look forward to in the Gaia data release based on the full extended mission!

\subsection{Synergies}

As laid out in Bland-Hawthorn \& Freeman (2000) and Freeman \& Bland-Hawthorn (2002), unravelling the formation history of the Milky Way can only be achieved by combining distance and kinematic information with knowledge of the detailed chemical compositions of the stars. Hence, while Gaia is central to the field of Galactic archaeology, its full power can only be unleashed by combining its data with other sky surveys. As summarized in Helmi (2020), the community has made a concerted effort since the approval of the Gaia mission in 2000 to propose, build, and operate large ground based spectroscopic surveys. These collect radial velocity data at the faint end of the Gaia survey $(G>15)$, and the crucial chemical composition information needed to untangle the formation history of the Milky Way. The combination of Gaia and photometric sky surveys opens up the way to distances and astrophysical parameters for all stars surveyed by Gaia, as demonstrated for example by Anders et al. (2019). Photometric surveys and Gaia are also a powerful combination when it comes to mapping the $3 \mathrm{D}$ distribution of interstellar dust as demonstrated by the works of Lallement et al. (2019), Green et al. (2019), and Hottier et al. (2020). The Vera Rubin Observatory's Legacy Survey of Space and Time (LSST, Ivezić et al. 2019) is around the corner, and like other surveys will profit from the astrometric and photometric reference system provided by Gaia. The synergies between LSST and Gaia were already anticipated by Ivezić et al. (2012). As illustrated in their Fig. 21, LSST will in some sense be the deep extension of Gaia from $r \sim 20$ to $r \sim 24$, with astrometric uncertainties that are anticipated to be comparable to Gaia performance at 19th magnitude and then smoothly extending the uncertainty vs. magnitude curve by roughly 5 mag. The stacked LSST images will reach depths of $r \sim 27$ meaning that LSST can peer deep into the outer halo of the Galaxy, resolving main sequence turn-off stars out to $300 \mathrm{kpc}$ (Ivezić et al. 2019). This is further motivation to build on the Gaia parallaxes to calibrate photometric distance indicators in order to turn the LSST imaging as accurately as possible into a deep spatial map.

\section{FUTURE DIRECTIONS FOR MICROARCSECOND ASTROMETRY}

In considering future directions for astrometry a number of options can be considered depending on the science case: increasing the precision and/or accuracy achieved for a given source brightness; extending a given precision level to fainter limits; extending a given precision to a different wavelength domain. A trade-off has to be made between the various 
options which also involves a decision between narrow- and wide-angle astrometry, with higher precision typically easier to achieve at narrow angles. An additional important consideration is the maintenance of the dense and highly accurate optical reference frame that Gaia provides. In the following I only briefly comment on ground based advances coming up and then focus on the future of space astrometry.

\subsection{Ground-based microarcsecond astrometry}

The efforts to achieve microarcsecond astrometry across the radio frequency domain, through technology and calibration method improvements, are discussed in Rioja \& Dodson (2019,2020). Microarcsecond astrometry is considered "routine" at 8 to $22 \mathrm{GHz}$ frequencies with good progress being made at higher frequencies. The prospect of the SKA is driving the developments to improve astrometric precision at low $\lesssim 8 \mathrm{GHz}$ frequencies, where systematic errors due to the effects of the ionosphere present the main challenge.

In the optical/IR domain on the ground GRAVITY is currently the only instrument capable of astrometry at the tens of microarcsecond level over narrow angles. Ireland \& Woillez (2018) argue that in principle the current generation of single optical/IR telescopes and long baseline interferometers can achieve astrometric precisions at the single $\mu$ as level, provided instruments are developed with sufficient control over and knowledge of the optical path taken by the astronomical light, as well as a sufficient knowledge of the astrometric (i.e. interferometer) baseline. It will be important to ensure that such instruments can access as much of the sky as possible for targets as faint as possible. The generation of giant optical/IR telescopes currently in the planning and construction phases will feature aperture sizes of up to $39 \mathrm{~m}$ in the case of the European Extremely Large Telescope (ELT). In principle this should allow for astrometry at the $\sim 50 \mu$ as level, provided image locations can be determined at the level of $0.5 \%$ of a resolution element and that various sources of systematic error can be overcome. The MICADO instrument under development for the ELT is aimed at reaching the $50 \mu$ as astrometric accuracy level over a 1 arcsec field of view (Rodeghiero et al. 2019, Trippe et al. 2010). This would for example allow the measurement of single-epoch tangential motions to $\sim 20 \mathrm{~km} \mathrm{~s}^{-1}$ at $100 \mathrm{kpc}$, and with a few years of observations would produce precisions at the $5 \mathrm{~km} \mathrm{~s}^{-1}$ level, sufficient to measure the internal kinematics in dwarf galaxies.

\subsection{Space astrometry prospects}

In the coming decade a number of space mission with astrometric capabilities will be launched (cf. Vallenari 2018). Of these the Small-JASMINE mission (Gouda 2020 ${ }^{10}$ is a dedicated space astrometry mission which will survey an area of $0.7^{\circ}$ radius around the Galactic centre as well as a region along the Galactic plane $\left(-2^{\circ}<l<0.7^{\circ}\right.$ and $\left.0^{\circ}<b<0.3^{\circ}\right)$ in the $H_{\mathrm{w}}$ band at $1.1-1.7 \mu \mathrm{m}$. The astrometric accuracies targeted are $25 \mu$ as and $25 \mu$ as $\mathrm{yr}^{-1}$ for parallaxes and proper motions, respectively, at $H_{\mathrm{w}}<12.5$. At $H_{\mathrm{w}}=15$ the accuracies are expected to be $\sim 125 \mu$ as. Both upcoming NASA space observatories, the James Webb Space Telescope and the Nancy Grace Roman Space Telescope, can be used for astrometry. For the latter an extensive science case was presented along with the expected astrometric performances (WFIRST Astrometry Working Group et al. 2019).

\footnotetext{
10 http://www.jasmine-galaxy.org/index.html
} 


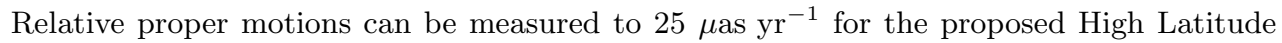
Survey, while astrometric precisions of 3-10 $\mu$ as are anticipated for relative astrometry of exoplanets or using the spatial scanning technique. Absolute astrometry to 0.1 mas can be achieved with the Roman Space Telescope by anchoring the measurements to the Gaia reference frame. The Gaia-CRF would be the limiting factor and WFIRST Astrometry Working Group et al. (2019) emphasize the significant benefits of an extended Gaia mission in terms of the reference frame accuracy at the time the Roman Space Telescope is operational.

On a longer timescale two white papers on space astrometry missions have been submitted to ESA as part its Voyage 2050 long-term planning process. The "Faint Objects in Motion" paper (Malbet et al. 2019) makes the case for narrow-angle astrometry from space, targeting end-of-mission accuracies from $10 \mu$ as at $R \sim 14-22$ for dark matter and compact object studies, down to $0.15 \mu$ as at $R \sim 1-18$ for exoplanet studies. The mission concept relies on a single $0.8 \mathrm{~m}$ telescope for which the PSF is about 0.2 arcsec in size. This implies that image location systematics must be under control to 1 part in 200000 in order to achieve the desired differential astrometric accuracy. This includes contributions from random noise sources, instabilities in the optical aberrations and focal plane geometry, and variations of detector quantum efficiency between pixels. The development of sophisticated metrology at the telescope and focal plane level is required. Much of that work has already been done in the context of the SIM PlanetQuest programme (Unwin et al. 2008), and as part of the preparations for the NEAT mission proposal (Malbet et al. 2012).

The other Voyage 2050 white paper concerns the GaiaNIR mission proposal (Hobbs et al. 2019). This mission would operate on the same principles as Gaia except for conducting its observations over a wide optical to near-infrared band (400-1800 nm). The science case is laid out in Hobbs et al. (2019) and is as broad as that for Gaia. Out to 20th magnitude some 8 billion sources would be observed, most of the gain being in the Galactic plane, where the dust limits the reach of Gaia. The resulting survey would on its own have an astrometric performance comparable to Gaia and allow for synergies with a host of other instruments in space and on the ground, in particular the large spectroscopic surveys in the infrared such as SDSS-V and MOONS. The dense sampling of a much larger volume over the Galactic disk would address one of the lessons learned from Section 4.2 A design study of the GaiaNIR concept was undertaken by ESA with the support of experts from the Gaia community and the resulting report is publicly available ${ }^{11}$. The major technology challenge for a revolving scanning mission in the infrared will be the development of corresponding TDI capable detectors. An alternative would be to include an optical element which would be capable of stabilizing images in the focal plane as the spacecraft rotates.

\subsection{Reference frame maintenance}

An essential element for the future of astrometry and the science it enables is the maintenance of the celestial reference frame. This frame is ultimately defined by the positions of the sources in an astrometric catalogue. The use of these positions at any given epoch $t_{\mathrm{o}}$ (to serve as a reference for astrometric observations) requires propagating them from the catalogue epoch $t_{\mathrm{c}}$ to $t_{\mathrm{o}}$. The reference frame as materialized by quasars will have a zero spin but with some uncertainty which propagates from $t_{\mathrm{c}}$ to $t_{\mathrm{o}}$. An additional uncertainty

\footnotetext{
${ }^{11}$ https://sci.esa.int/s/8a65kZA
} 


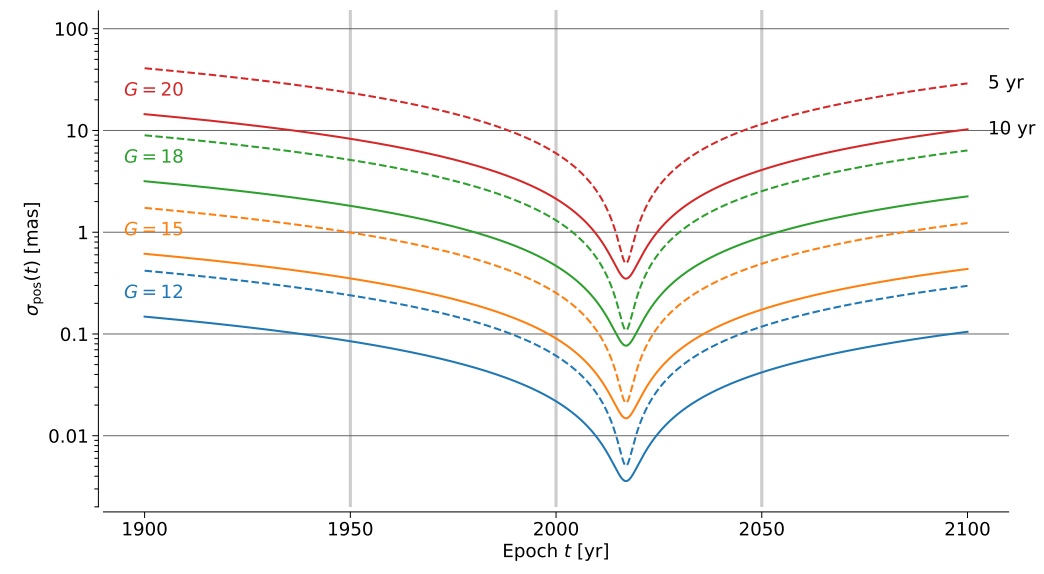

Figure 10

The evolution of the Gaia source position uncertainties over time. The curves show how the uncertainty on the celestial position of a source (to be precise, the semi-major axis of the position uncertainty ellipse as defined by Eq. B.1 in Lindegren et al. 2018) degrades over time as one moves away from the mid-epoch of the mission. This is caused mainly by the uncertainty in the proper motions. The curves shows the uncertainty degradation for 4 different magnitudes and for the 5-yr (dashed lines) and 10-yr (solid lines) mission life times. Credits: Figure adapted from Hobbs et al. (2016), right panel of their Fig. 4 which was created by F. Mignard.

is introduced by the errors on the determination of the acceleration of the solar system barycentre relative to the quasar rest frame. The acceleration is manifest as a proper motion pattern in the quasars, and uncertainties therein also propagate into future positions.

In practice however, the Gaia-CRF will most often be used by anchoring new astrometric observations to the Gaia positions of stars. The stars provide a much denser reference frame over a larger range in brightness, making a larger area of the sky accessible to (relative) astrometry. The degradation of the stellar reference frame is dominated by the uncertainties at $t_{\mathrm{c}}$ in the proper motions of the stars (the rigorous propagation of astrometric parameters also depends on the parallax and radial velocity, e.g., Butkevich \& Lindegren 2014), leading to a degradation in time of the positional uncertainties, roughly proportional to $t_{\mathrm{o}}-t_{\mathrm{c}}$. This is illustrated for the case of the Gaia in Figure 10 At the faint end of the Gaia survey the typical positional uncertainty of $\sim 0.5$ mas at the end of the 5 -yr mission grows to about 3 mas by 2025 and 10 mas by 2050. To put these numbers in context, WFIRST Astrometry Working Group et al. (2019) state that Gaia catalogue position errors of a few mas translate to $\sim 0.02$ pixel errors in the Roman Space Telescope Wide Field Instrument, which significantly impacts the ability to perform high precision absolute astrometry (i.e. to better then 0.01 WFI pixel). Figure 10 also shows the positional uncertainties over time in case the Gaia mission life time is extended to 10 years. The factor 2.8 improvement in the proper motions leads to a stellar reference frame accurate to 1 mas in 2025 and 4 mas in 2050. This would thus be of tremendous benefit for high precision absolute astrometry with other space or ground based instruments. For example the MICADO team investigated the use of Gaia stars to aid in calibrating out the effects of optical distortions on the astrometry (Rodeghiero et al. 2018), an effort that will again benefit from the best possible accuracy for a network of faint star positions. Figure 10 is deliberately extended into the past to 
illustrate the fact that old observations can also be re-reduced on the Gaia reference frame, which remains better than anything available in the past even over more than a century. As mentioned in Section 4.1.1 this is used for example to improve the orbits of asteroids from position measurements over long time spans.

Improving the reference frame by extending the mission life time of Gaia points to a very important benefit of the GaiaNIR proposal. It would enable the combination of two independent astrometric surveys to derive proper motion measurements over a time baseline of 20 years which leads to a factor of 20 improvement in the proper motion precision Hobbs et al. 2019). The stellar reference frame would both improve in precision and degrade much more slowly (positional uncertainties remaining below $\sim 1-5$ mas out to 2100 as shown in Fig. 3 of Hobbs et al. 2019). The improved proper motions are scientifically interesting by themselves and would also lead to improved parallaxes for the stars in common between GaiaNIR and Gaia.

Apart from the science enabled by the dense and accurate stellar reference frame, maintenance and improvement of the celestial reference frame as defined by the quasars is also of great scientific interest. It is well known that quasars show variability in their optical positions which can be attributed to the internal source structure variations (Taris et al. 2011), which can lead to apparent non-zero parallaxes and proper motions. However, even if the positions would be perfectly stable, quasar proper motion patterns would still appear in high precision astrometric surveys because the solar system barycentre is not at rest with respect to cosmological microwave background. Bachchan et al. (2016) review these proper motion patterns and how they might be measured from the Gaia data. The most prominent proper motion pattern is due to the acceleration of the barycentre and its

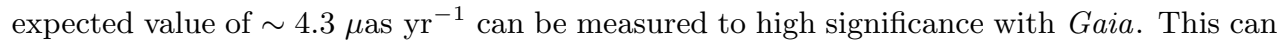
be done by solving for the acceleration parameters as part of the overall reference frame determination (where for Gaia DR2 only orientation and spin were considered). The instantaneous velocity of the solar system with respect to he CMB will cause a quasar proper

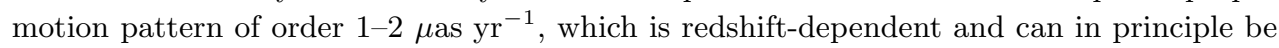
used to measure the Hubble constant. Bachchan et al. (2016) show that this is at the limit of the possibilities with Gaia, but in combination with a mission like GaiaNIR this would become a secure measurement. Further examples of proper motion patterns are those due to gravitational waves with periods longer than the Gaia mission length, and changes in the angular separation between distant sources due to a possibly non-isotropic expansion of the universe. In the case of gravitational waves, upper limits can be measured for their energy flux at frequencies below $1.5 \mathrm{nHz}$, where Gaia alone is expected to improve on existing limits by 1 to 2 orders of magnitude (Klioner 2014, Mignard \& Klioner 2012). A combined Gaia-GaiaNIR measurement would provide another factor 400 improvement (Hobbs et al. 2019 ). The anisotropy of expansion causes proper motion effects at the $\sim 0.02-2 \mu \mathrm{as} \mathrm{yr}^{-1}$ level, which can only be measured with a Gaia-GaiaNIR combination.

\subsection{Splitting the microarcsecond}

The SIM PlanetQuest Programme as well as the NEAT proposal and the white paper by Malbet et al. (2019) all aim for sub- $\mu$ as accuracy for narrow-angle astrometry. In the context of the definition of science themes for the L2 and L3 missions in ESA's Cosmic Vision Programme, the white paper "Space-Time Structure Explorer: Sub-microarcsecond astrometry for the 2030s" explored the science cases and the challenges of an all-sky absolute 
astrometry mission aiming for the sub- $\mu$ as to nano-arcsecond regime (Brown 2013). Fig. 1 in Brown (2014) illustrates that such a mission would allow the direct measurement of percent level parallaxes and a few $\mathrm{km} \mathrm{s}^{-1}$ tangential motions for bright tracer populations out to 0.1-1 Mpc. Such a mission concept faces several major engineering and data processing challenges. Assuming an optical/IR mission, the efficiency of current telescope/detector technology is already close to $100 \%$. The basic scaling relation for the precision (Equation 1 ) then shows that major gains in accuracy can only be obtained through increases in the aperture or baseline $B$. Continuing this line of reasoning, one quickly ends up with an argument for an interferometric mission with collecting areas of a few $\mathrm{m}^{2}$ and baselines of order 100-1000 m (see also Lindegren 2007). This implies the development of precision formation flying, which is already being developed and tested in the context of the PROBA3 and e-LISA missions, as well as elaborating an operations concept that would allow for absolute astrometry. Further engineering challenges include the extreme requirements on the thermo-mechanical stability of the spacecraft structures, on the attitude control, and on the knowledge of the barycentric velocity of the spacecraft. The challenges mentioned above for the narrow angle astrometry missions at the level of detector and optical distortion effects also apply here.

Data processing to achieve sub- $\mu$ as precisions will be complicated by (among others):

- Relativistic modelling of astrometric measurements will have to be pushed to the nano-arcsec level in order to correctly interpret the raw data. Going beyond $0.1 \mu$ as precision in relativistic modelling would require research as well as improvements in our knowledge of the solar system (asteroid masses for example).

- System calibration at this level will have to be much better (by an order of magnitude) than the astrometric accuracy aimed for and will thus be extremely challenging. The design of the instruments and mission concept will have to incorporate the data processing demands from the start. That is, any mission study and development phases should treat engineering and data processing on an equal footing. This is especially important where for example it is clear that certain stability requirements cannot be met through engineering but may be managed through a combination of metrology and sophisticated calibration concepts at data processing level.

- Approaching nano-arcsecond levels it is not obvious that simple models of the time dependence of source coordinates will be sufficient. In addition research into sources of astrometric jitter (such as star spots or microlensing in crowded regions) and their effect on the interpretation of image locations in the data stream is required.

- Another conceptual problem when approaching the nano-arcsecond regime is that for parallax measurements small $(<1 \mathrm{au})$ sources which are sufficiently bright (hot) are required in large numbers (for global astrometry). It is not clear that at cosmological distances this requirement is fulfilled (Lindegren 2007).

\section{CONCLUSIONS}

\section{SUMMARY POINTS}

1. Gaia is revolutionizing astronomy through the vast set of fundamental astronomical data that touches on every science topic. In parallel the celestial reference frame 
provided by Gaia enables the accurate astrometric and photometric calibration of past, current, and future sky surveys. Astronomical research and observations will profit from this legacy for many decades to come.

2. Gaia DR2 definitively demonstrated the power of an all-sky, high spatial resolution, high astrometric and photometric accuracy survey, enabling a vastly improved open cluster census, the tracing of stellar streams all across the sky, and fully mapping nearby satellite galaxies as well as the outer regions of globular clusters.

3. Gaia DR2 for the first time provides a dense sampling of Galactic phase space at high astrometric, photometric, and radial velocity precisions, which is essential for uncovering the subtle features in phase space as well as in the observational HR-diagram that are leading to new insights into the Milky Way and stars.

4. Gaia DR2 provides exquisite astrometry which nevertheless suffers from systematic errors. Armed with a detailed understanding of Gaia measurements and the data processing, efforts are underway to ensure that systematic errors remain well below the increasing precision levels of future data releases.

5. Continuing down the path of microarcsecond optical/infrared absolute astrometric surveys is only feasible from space, and is required for the maintenance of the celestial reference frame which will anchor the ground based photometric and spectroscopic surveys as well as the extremely large aperture facilities of the future.

6. The 21st century astronomer has an array of microarcsecond astrometry facilities at their disposal, with VLBI, GRAVITY, and HST providing vital capabilities in dust obscured and crowded sky regions where Gaia's capabilities are limited.

Future efforts remain necessary both to maximize the scientific exploitation of data collected by the Gaia mission but also to extend the microarcsecond astrometric survey capabilities into the future and possibly go one step beyond to the nanoarcsecond regime.

\section{FUTURE ISSUES}

1. Achieving the highest possible accuracies at the bright end of the Gaia survey will remain challenging. Complementary VLBI astrometry of optically bright radio stars will remain essential as validation of the Gaia astrometry, and for accurately characterizing the bright Gaia reference frame. It is important that VLBI astrometry publications provide full astrometric solutions, including celestial positions and their observation epochs.

2. The true power of Gaia lies in the combination of its data with photometric and spectroscopic surveys. The planning for future surveys should take into account the need to acquire the optimal mix of fundamental astronomical data at compatible accuracy levels.

3. Determining the Gaia survey selection function is essential to unlocking some of the key science cases and will require a major research effort from the community, which should be supported by the release of as yet unpublished information relevant to the Gaia selection function. Future large survey plans should include from the very start the task to build and provide a survey selection function.

4. Taking full advantage of the precision of Gaia proper motions out to large distances 
requires using the Gaia parallaxes to advance the precision of alternative distance indicators, especially photometric distance indicators across the HR diagram.

5. As a next space astrometry mission the GaiaNIR option is the most feasible and will offer the widest set of science cases, the advantage of a dense sampling of the Milky Way disk complementing upcoming infrared spectroscopic surveys, while enabling a reset of optical reference frame accuracies to milliarcsecond levels into the next century and providing an unprecedented infrared reference frame.

6. Nevertheless Gaia astrometry is likely to remain the standard for decades to come, including its treasure trove of astrometric, spectrophotometric, and spectroscopic time series. It is thus essential to invest effort into the long-term preservation of the Gaia data archive to keep it easily interoperable with other surveys and to enable the community to mine the epoch data and reprocess intermediate level Gaia data products in the light of new complementary data.

7. Entering the nanoarcsecond absolute astrometry domain requires rethinking the Hipparcos/Gaia concept at several levels: the engineering challenges of wide-angle astrometry with a space interferometric system; the modelling of source motions; solving the issue of a lack of compact sources for parallax measurements over cosmological distances; and the modelling of light propagation in the presence of solar system light bending effects at the sub-nanoarcsecond level.

\section{DISCLOSURE STATEMENT}

The author is not aware of any affiliations, memberships, funding, or financial holdings that might be perceived as affecting the objectivity of this review.

\section{ACKNOWLEDGMENTS}

It is a great pleasure to thank the many DPAC and ESA colleagues as well as the Airbus Space engineer team, for providing me over the years with their wisdom and insights that contributed in one way or another to this review taking shape. It is their fantastic efforts over the past 25 years that have made Gaia a reality and incredible success. In particular I wish to thank Paolo Tanga, Timo Prusti, and Jos de Bruijne for valuable inputs and comments on early drafts. This work has made use of data from the European Space Agency (ESA) mission Gaia (https://www.cosmos.esa.int/gaia), processed by the Gaia Data Processing and Analysis Consortium (DPAC, https://www.cosmos.esa. int/web/gaia/dpac/consortium). Funding for the DPAC has been provided by national institutions, in particular the institutions participating in the Gaia Multilateral Agreement. Funding from the Netherlands Organisation for Scientific Research (NWO) through grant NWO-M-614.061.414 and the Netherlands Research School for Astronomy (NOVA) is gratefully acknowledged. This work made use of the following software: Astropy, a communitydeveloped core Python package for Astronomy (Astropy Collaboration et al. 2013, 2018, http://www.astropy.org), IPython (Pérez \& Granger 2007, https://ipython.org/), Jupyter (https://jupyter.org/), Matplotlib (Hunter 2007, https://matplotlib.org), SciPy (Virtanen et al. 2020, https://www.scipy.org), NumPy (Harris et al. 2020 https: //numpy.org), scikit-learn (Pedregosa et al. 2011, https://scikit-learn.org/stable/) 
and TOPCAT (Taylor 2005, http://www.starlink.ac.uk/topcat/). This work has made use of NASA's Astrophysics Data System.

\section{LITERATURE CITED}

Agnello A, Lin H, Kuropatkin N, Buckley-Geer E, Anguita T, et al. 2018. MNRAS 479:4345-4354 Altmann M, Bouquillon S, Taris F, Steele IA, Smart RL, et al. 2014. GBOT: ground based optical tracking of the Gaia satellite. In Observatory Operations: Strategies, Processes, and Systems V, vol. 9149 of Society of Photo-Optical Instrumentation Engineers (SPIE) Conference Series

Altmann M, Roeser S, Demleitner M, Bastian U, Schilbach E. 2017. A\&A 600:L4

Anders F, Cantat-Gaudin T, Quadrino-Lodoso I, Gieles M, Jordi C, et al. 2020. arXiv e-prints :arXiv:2006.01690

Anders F, Khalatyan A, Chiappini C, Queiroz AB, Santiago BX, et al. 2019. A\&A 628:A94

Anderson L, Hogg DW, Leistedt B, Price-Whelan AM, Bovy J. 2018. AJ 156:145

Andrae R, Fouesneau M, Creevey O, Ordenovic C, Mary N, et al. 2018. A\&SA 616:A8

Andrews SM, Huang J, Pérez LM, Isella A, Dullemond CP, et al. 2018. ApJ 869:L41

Antoja T, Helmi A, Romero-Gómez M, Katz D, Babusiaux C, et al. 2018. Nature 561:360-362

Antoja T, Ramos P, Mateu C, Helmi A, Anders F, et al. 2020. A 8 A 635:L3

Arai T, Yoshida F, Hong P, Okamoto N, Noda H, et al. 2020. Stellar Occultation Observations of Asteroid (3200) Phaethon for the DESTINY+ Flyby. In 51st Lunar and Planetary Science Conference, Lunar and Planetary Science Conference

Arenou F, Luri X, Babusiaux C, Fabricius C, Helmi A, et al. 2017. A\& A 599:A50

Arenou F, Luri X, Babusiaux C, Fabricius C, Helmi A, et al. 2018. A\&SA 616:A17

Arimatsu K, Hashimoto GL, Kagitani M, Sakanoi T, Kasaba Y, et al. 2020. A\&A 638:L5

Astropy Collaboration, Price-Whelan AM, Sipőcz BM, Günther HM, Lim PL, et al. 2018. AJ 156:123

Astropy Collaboration, Robitaille TP, Tollerud EJ, Greenfield P, Droettboom M, et al. 2013. A\& A 558:A33

Bachchan RK, Hobbs D, Lindegren L. 2016. A\& A 589:A71

Bastian U, Biermann M. 2005. A\&A 438:745-755

Bauer EB, Schwab J, Bildsten L, Cheng S. 2020. arXiv e-prints :arXiv:2009.04025

Bellini A, Vesperini E, Piotto G, Milone AP, Hong J, et al. 2015. ApJ 810:L13

Belokurov V, Erkal D, Deason AJ, Koposov SE, De Angeli F, et al. 2017. MNRAS 466:4711-4730

Belokurov V, Penoyre Z, Oh S, Iorio G, Hodgkin S, et al. 2020. MNRAS 496:1922-1940

Benedict GF, McArthur BE, Nelan EP, Harrison TE. 2017. PASP 129:012001

Bennett M, Bovy J. 2019. MNRAS 482:1417-1425

Berger TA, Huber D, Gaidos E, van Saders JL. 2018. ApJ 866:99

Berger TA, Huber D, Gaidos E, van Saders JL, Weiss LM. 2020a. AJ 160:108

Berger TA, Huber D, van Saders JL, Gaidos E, Tayar J, Kraus AL. 2020b. AJ 159:280

Bergeron P, Dufour P, Fontaine G, Coutu S, Blouin S, et al. 2019. ApJ 876:67

Bianchini P, van der Marel RP, del Pino A, Watkins LL, Bellini A, et al. 2018. MNRAS 481:21252139

Binney J, Schönrich R. 2018. MNRAS 481:1501-1506

Bland-Hawthorn J, Freeman K. 2000. Science 287:79

Bland-Hawthorn J, Gerhard O. 2016. ARA 8 A 54:529-596

Bland-Hawthorn J, Sharma S, Tepper-Garcia T, Binney J, Freeman KC, et al. 2019. MNRAS 486:1167-1191

Bland-Hawthorn J, Tepper-Garcia T. 2020. arXiv e-prints :arXiv:2009.02434

Bombrun A, Lindegren L, Holl B, Jordan S. 2010. A 6 A 516:A77

Bonaca A, Conroy C, Hogg DW, Cargile PA, Caldwell N, et al. 2020. ApJ 892:L37

Bonaca A, Hogg DW, Price-Whelan AM, Conroy C. 2019. ApJ 880:38 
Bossini D, Vallenari A, Bragaglia A, Cantat-Gaudin T, Sordo R, et al. 2019. A\&A 623:A108

Boubert D, Everall A. 2020. MNRAS 497:4246-4261

Boubert D, Everall A, Holl B. 2020. MNRAS 497:1826-1841

Boubert D, Strader J, Aguado D, Seabroke G, Koposov SE, et al. 2019. MNRAS 486:2618-2630

Bouy H, Alves J. 2015. A\&A 584:A26

Brandt TD. 2018. ApJS 239:31

Brandt TD, Dupuy TJ, Bowler BP. 2019. AJ 158:140

Brown A. 2013. White Paper - Space-Time Structure Explorer Sub-microarcsecond astrometry for the 2030s. GAIA-CG-TN-LEI-AB-039

Brown AGA. 2010. ELSA and the Frontiers of Astrometry. In Gaia: At the Frontiers of Astrometry, eds. C Turon, F Meynadier, F Arenou, vol. 45 of EAS Publications Series

Brown AGA. 2012. Science from Gaia: How to Deal with a Complex Billion-Source Catalogue and Data Archive. In Astrostatistics and Data Mining, eds. LM Sarro, L Eyer, W O'Mullane, J De Ridder, vol. 2 of Springer Series in Astrostatistics. Spinger

Brown AGA. 2014. Space astrometry in the Gaia era and beyond. In The Milky Way Unravelled by Gaia: GREAT Science from the Gaia Data Releases, eds. N Walton, F Figueras, L BalaguerNuñez, C Soubiran, vol. 67-68 of EAS Publications Series

Brown AGA. 2018. The Gaia sky: version 1.0. In Astrometry and Astrophysics in the Gaia Sky, eds. A Recio-Blanco, P de Laverny, AGA Brown, T Prusti, vol. 330 of IAU Symposium

Buder S, Asplund M, Duong L, Kos J, Lind K, et al. 2018. MNRAS 478:4513-4552

Buie MW, Porter SB, Tamblyn P, Terrell D, Parker AH, et al. 2020. AJ 159:130

Butkevich AG, Klioner SA, Lindegren L, Hobbs D, van Leeuwen F. 2017. A\&A 603:A45

Butkevich AG, Lindegren L. 2014. A\& A 570:A62

Callingham TM, Cautun M, Deason AJ, Frenk CS, Wang W, et al. 2019. MNRAS 484:5453-5467 Cantat-Gaudin T, Anders F. 2020. A\& A 633:A99

Cantat-Gaudin T, Anders F, Castro-Ginard A, Jordi C, Romero-Gómez M, et al. 2020. AछA 640:A1

Cantat-Gaudin T, Jordi C, Vallenari A, Bragaglia A, Balaguer-Núñez L, et al. 2018a. A $\& A$ 618:A93

Cantat-Gaudin T, Krone-Martins A, Sedaghat N, Farahi A, de Souza RS, et al. 2019. A $\& A$ 624:A126

Cantat-Gaudin T, Vallenari A, Sordo R, Pensabene F, Krone-Martins A, et al. 2018b. A\&A 615:A49

Carraro G. 2018. Research Notes of the American Astronomical Society 2:12

Casertano S, Riess AG, Anderson J, Anderson RI, Bowers JB, et al. 2016. ApJ 825:11

Castro-Ginard A, Jordi C, Luri X, Cantat-Gaudin T, Balaguer-Núñez L. 2019. A 8 A 627:A35

Castro-Ginard A, Jordi C, Luri X, Julbe F, Morvan M, et al. 2018. A\&A 618:A59

Chan VC, Bovy J. 2020. MNRAS 493:4367-4381

Cheng S, Cummings JD, Ménard B. 2019. ApJ 886:100

Chiavassa A, Freytag B, Schultheis M. 2018. A\&\&A 617:L1

Chiavassa A, Pasquato E, Jorissen A, Sacuto S, Babusiaux C, et al. 2011. A $\& A$ 528:A120

Choi J, Dotter A, Conroy C, Cantiello M, Paxton B, Johnson BD. 2016. ApJ 823:102

Clementini G, Ripepi V, Leccia S, Mowlavi N, Lecoeur-Taibi I, et al. 2016. A $\& A$ 595:A133

Cloutier R, Eastman JD, Rodriguez JE, Astudillo-Defru N, Bonfils X, et al. 2020. AJ 160:3

Counselman C. C. I. 1976. ARA\&A 14:197-214

Cranmer MD, Galvez R, Anderson L, Spergel DN, Ho S. 2019. arXiv e-prints :arXiv:1908.08045

Cropper M, Katz D, Sartoretti P, Prusti T, de Bruijne JHJ, et al. 2018. AESA 616:A5

Crowley C, Kohley R, Hambly NC, Davidson M, Abreu A, et al. 2016. A\&3 A 595:A6

Curtis JL, Agüeros MA, Mamajek EE, Wright JT, Cummings JD. 2019. AJ 158:77

Darling K, Widrow LM. 2019. MNRAS 484:1050-1056

de Boer TJL, Erkal D, Gieles M. 2020. MNRAS 494:5315-5332

de Bruijne JHJ, Allen M, Azaz S, Krone-Martins A, Prod'homme T, Hestroffer D. 2015. A\& A 576:A74

Deason AJ, Belokurov V, Koposov SE, Gómez FA, Grand RJ, et al. 2017. MNRAS 470:1259-1273

Delbo' M, Gayon-Markt J, Busso G, Brown A, Galluccio L, et al. 2012. Planet. Space Sci. 73:86-94 
Delchambre L, Krone-Martins A, Wertz O, Ducourant C, Galluccio L, et al. 2019. A 8 A 622:A165 Desmars J, Bancelin D, Hestroffer D, Thuillot W. 2013. Astronomy E Astrophysics 554:A32 Desmars J, Meza E, Sicardy B, Assafin M, Camargo JIB, et al. 2019a. A\&GA 625:A43

Desmars J, Sicardy B, Braga-Ribas F, Benedetti-Rossi G, Marques Oliveira J, et al. 2019b. Prediction of stellar occultations by distant Solar System objects with Gaia. In SF2A-2019: Proceedings of the Annual meeting of the French Society of Astronomy and Astrophysics, eds. P Di Matteo, O Creevey, A Crida, G Kordopatis, J Malzac, JB Marquette, M N'Diaye, O Venot

Dotter A. 2016. ApJS 222:8

Ducourant C, Wertz O, Krone-Martins A, Teixeira R, Le Campion JF, et al. 2018. A\&SA 618:A56

Dupuy TJ, Brandt TD, Kratter KM, Bowler BP. 2019. ApJ 871:L4

Eadie G, Jurić M. 2019. ApJ 875:159

El-Badry K, Rix HW, Weisz DR. 2018. ApJ 860:L17

Erkal D, Belokurov V, Laporte CFP, Koposov SE, Li TS, et al. 2019. MNRAS 487:2685-2700

ESA, ed. 1997. The HIPPARCOS and TYCHO catalogues. Astrometric and photometric star catalogues derived from the ESA HIPPARCOS Space Astrometry Mission, vol. 1200 of ESA Special Publication

Ester M, peter Kriegel H, Sander J, Xu X. 1996. A density-based algorithm for discovering clusters in large spatial databases with noise. In Proceedings of the Second International Conference on Knowledge Discovery and Data Mining, eds. E Simoudis, J Han, U Fayyad. AAAI Press

Evans DW, Riello M, De Angeli F, Carrasco JM, Montegriffo P, et al. 2018. A\&A 616:A4

Fabricius C, Bastian U, Portell J, Castañeda J, Davidson M, et al. 2016. A\& A 595:A3

Feissel M, Mignard F. 1998. A\&3A 331:L33-L36

Fienga A, Manche H, Laskar J, Gastineau M, Verma A. 2013. arXiv e-prints :arXiv:1301.1510

Freeman K, Bland-Hawthorn J. 2002. ARA\&A 40:487-537

Fricke W, Schwan H, Lederle T, Bastian U, Bien R, et al. 1988. Veroeffentlichungen des Astronomischen Rechen-Instituts Heidelberg 32:1-106

Fritz TK, Battaglia G, Pawlowski MS, Kallivayalil N, van der Marel R, et al. 2018. A $\& A$ 619:A103

Fulton BJ, Petigura EA. 2018. AJ 156:264

Fürnkranz V, Meingast S, Alves J. 2019. A\&A 624:L11

Gaia Collaboration, Babusiaux C, van Leeuwen F, Barstow MA, Jordi C, et al. 2018a. A\&A 616:A10

Gaia Collaboration, Brown A, Vallenari A, Prusti T, de Bruijne JHJ, et al. 2020. submitted to A\&A

Gaia Collaboration, Brown AGA, Vallenari A, Prusti T, de Bruijne JHJ, et al. 2016a. A\&SA 595:A2

Gaia Collaboration, Brown AGA, Vallenari A, Prusti T, de Bruijne JHJ, et al. 2018b. A\&A 616:A1

Gaia Collaboration, Eyer L, Rimoldini L, Audard M, Anderson RI, et al. 2019. A\& A 623:A110

Gaia Collaboration, Helmi A, van Leeuwen F, McMillan PJ, Massari D, et al. 2018c. A 6 A 616:A12

Gaia Collaboration, Katz D, Antoja T, Romero-Gómez M, Drimmel R, et al. 2018d. A $\& A$ 616:A11

Gaia Collaboration, Mignard F, Klioner SA, Lindegren L, Hernández J, et al. 2018e. A $\& A$ 616:A14

Gaia Collaboration, Prusti T, de Bruijne JHJ, Brown AGA, Vallenari A, et al. 2016b. A 8 A 595:A1

Gaia Collaboration, Spoto F, Tanga P, Mignard F, Berthier J, et al. 2018f. A\&\&A 616:A13

Gentile Fusillo NP, Gänsicke BT, Greiss S. 2015. MNRAS 448:2260-2274

Gentile Fusillo NP, Tremblay PE, Gänsicke BT, Manser CJ, Cunningham T, et al. 2019. MNRAS 482:4570-4591

Gielesen W, de Bruijn D, van den Dool T, Kamphues F, Mekking J, et al. 2013. Gaia basic angle monitoring system. In Cryogenic Optical Systems and Instruments 2013, eds. JB Heaney, ET Kvamme, vol. 8863. International Society for Optics and Photonics, SPIE

Gouda N. 2020. Infrared space astrometry mission for survey of the Galactic nuclear bulge: SmallJASMINE. In IAU Symposium 353: Galactic Dynamics in the Era of Large Surveys, eds. M Valluri, JA Sellwood, vol. 14 of Proceedings of the International Astronomical Union. Cambridge University Press

Gravity Collaboration, Abuter R, Accardo M, Amorim A, Anugu N, et al. 2017. A\&A 602:A94

Gravity Collaboration, Abuter R, Amorim A, Bauböck M, Berger JP, et al. 2019. A\&A 625:L10 
Green GM, Schlafly E, Zucker C, Speagle JS, Finkbeiner D. 2019. ApJ 887:93

Hall OJ, Davies GR, Elsworth YP, Miglio A, Bedding TR, et al. 2019. MNRAS 486:3569-3585

Harris CR, Millman KJ, van der Walt SJ, Gommers R, Virtanen P, et al. 2020. Nature 585:357-362

Helmi A. 2020. Annual Review of Astronomy and Astrophysics 58:null

Helmi A, Veljanoski J, Breddels MA, Tian H, Sales LV. 2017. A\&A 598:A58

Hobbs D, Brown A, Høg E, Jordi C, Kawata D, et al. 2019. arXiv e-prints :arXiv:1907.12535

Hobbs D, Høg E, Mora A, Crowley C, McMillan P, et al. 2016. arXiv e-prints :arXiv:1609.07325

Holl B, Audard M, Nienartowicz K, Jevardat de Fombelle G, Marchal O, et al. 2018. A\&A 618:A30

Hollands MA, Koester D, Alekseev V, Herbert EL, Gänsicke BT. 2017. MNRAS 467:4970-5000

Hottier C, Babusiaux C, Arenou F. 2020. arXiv e-prints :arXiv:2007.03734

Hunter JD. 2007. Computing in Science and Engineering 9:90-95

Ibata R, Thomas G, Famaey B, Malhan K, Martin N, Monari G. 2020. ApJ 891:161

Ibata RA, Bellazzini M, Malhan K, Martin N, Bianchini P. 2019a. Nature Astronomy 3:667-672

Ibata RA, Malhan K, Martin NF. 2019b. ApJ 872:152

Ireland MJ, Woillez J. 2018. arXiv e-prints :arXiv:1812.02926

Ivezić Ž, Beers TC, Jurić M. 2012. ARA $\& A$ 50:251-304

Ivezić Ž, Kahn SM, Tyson JA, Abel B, Acosta E, et al. 2019. ApJ 873:111

Jao WC, Feiden GA. 2020. AJ 160:102

Jao WC, Henry TJ, Gies DR, Hambly NC. 2018. ApJ 861:L11

Jiménez-Esteban FM, Torres S, Rebassa-Mansergas A, Skorobogatov G, Solano E, et al. 2018. MNRAS 480:4505-4518

Johnston KJ, de Vegt C. 1999. ARA\&SA 37:97-125

Jordan S, Bastian U, Lenhardt H, Bernstein HH, Hirte S, Biermann M. 2005. Gaia First Look. In The Three-Dimensional Universe with Gaia, eds. C Turon, KS O'Flaherty, MAC Perryman, vol. 576 of ESA Special Publication

Kallivayalil N, van der Marel RP, Alcock C, Axelrod T, Cook KH, et al. 2006. ApJ 638:772-785

Kamdar H, Conroy C, Ting YS, Bonaca A, Johnson B, Cargile P. 2019. ApJ 884:173

Katz D, Sartoretti P, Cropper M, Panuzzo P, Seabroke GM, et al. 2019. A\&SA 622:A205

Kervella P, Arenou F, Mignard F, Thévenin F. 2019. A $\& A$ 623:A72

Khan S, Miglio A, Mosser B, Arenou F, Belkacem K, et al. 2019. A\&3A 628:A35

Kharchenko NV, Piskunov AE, Schilbach E, Röser S, Scholz RD. 2013. A $6 A$ 558:A53

Kilic M, Hambly NC, Bergeron P, Genest-Beaulieu C, Rowell N. 2018. MNRAS 479:L113-L117

Klioner SA. 2003. AJ 125:1580-1597

Klioner SA. 2004. Phys. Rev. D 69:124001

Klioner SA. 2014. Gaia Astrometry and Fundamental Physics. In The Milky Way Unravelled by Gaia: GREAT Science from the Gaia Data Releases, eds. N Walton, F Figueras, L BalaguerNuñez, C Soubiran, vol. 67-68 of EAS Publications Series

Koch A, Hansen TT, Kunder A. 2018. A\&A 609:A13

Koposov SE, Belokurov V, Li TS, Mateu C, Erkal D, et al. 2019. MNRAS 485:4726-4742

Koposov SE, Belokurov V, Torrealba G. 2017. MNRAS 470:2702-2709

Kos J, de Silva G, Buder S, Bland -Hawthorn J, Sharma S, et al. 2018. MNRAS 480:5242-5259

Kounkel M, Covey K. 2019. AJ 158:122

Kounkel M, Covey K, Suárez G, Román-Zúñiga C, Hernandez J, et al. 2018. AJ 156:84

Kovalevsky J, Falin JL, Pieplu JL, Bernacca PL, Donati F, et al. 1992. A $\& A$ 258:7-17

Krone-Martins A, Delchambre L, Wertz O, Ducourant C, Mignard F, et al. 2018. A\&\&A 616:L11

Krone-Martins A, Ducourant C, Teixeira R, Galluccio L, Gavras P, et al. 2013. A $8 A$ 556:A102

Krone-Martins A, Moitinho A. 2014. A\&A 561:A57

Krumholz MR, McKee CF, Bland -Hawthorn J. 2019. ARA\& A 57:227-303

Lallement R, Babusiaux C, Vergely JL, Katz D, Arenou F, et al. 2019. A\&SA 625:A135

Lamers HJGLM, Gieles M, Bastian N, Baumgardt H, Kharchenko NV, Portegies Zwart S. 2005. A\&SA 441:117-129 
Laporte CFP, Johnston KV, Gómez FA, Garavito-Camargo N, Besla G. 2018. MNRAS 481:286-306 Laporte CFP, Minchev I, Johnston KV, Gómez FA. 2019. MNRAS 485:3134-3152

Leistedt B, Hogg DW. 2017. AJ 154:222

Leiva R, Sicardy B, Camargo JIB, Ortiz JL, Desmars J, et al. 2017. AJ 154:159

Lindegren L. 1980. A\&A 89:41-47

Lindegren L. 2005. The Astrometric Instrument of Gaia: Principles. In The Three-Dimensional Universe with Gaia, eds. C Turon, KS O'Flaherty, MAC Perryman, vol. 576 of ESA Special Publication

Lindegren L. 2007. Highlights of Astronomy 14:481-481

Lindegren L. 2018. Re-normalising the astrometric chi-square in Gaia DR2. GAIA-C3-TN-LU-LL124

Lindegren L. 2020a. $A \mathscr{S} A$ 633:A1

Lindegren L. 2020b. A\&A 637:C5

Lindegren L, Bastian U. 2010. Basic principles of scanning space astrometry. In Gaia: At the Frontiers of Astrometry, eds. C Turon, F Meynadier, F Arenou, vol. 45 of EAS Publications Series

Lindegren L, Hernández J, Bombrun A, Klioner S, Bastian U, et al. 2018. A\&A 616:A2

Lindegren L, Høg E, van Leeuwen F, Murray CA, Evans DW, et al. 1992. A $\& A$ 258:18-30

Lindegren L, Klioner S, Hernández J, Bombrun A, Ramos-Lerate M, et al. 2020. submitted to A\&̧A

Lindegren L, Kovalevsky J. 1995. A\&\&A 304:189

Lindegren L, Lammers U, Bastian U, Hernández J, Klioner S, et al. 2016. A 6 A 595:A4

Lindegren L, Lammers U, Hobbs D, O’Mullane W, Bastian U, Hernández J. 2012. A $\&$ A 538:A78

Liu L, Pang X. 2019. ApJS 245:32

Luri X, Brown AGA, Sarro LM, Arenou F, Bailer-Jones CAL, et al. 2018. A $\& A$ 616:A9

Ma C, Arias EF, Eubanks TM, Fey AL, Gontier AM, et al. 1998. AJ 116:516-546

Malbet F, Abbas U, Alves J, Boehm C, Brown W, et al. 2019. arXiv e-prints :arXiv:1910.08028

Malbet F, Léger A, Shao M, Goullioud R, Lagage PO, et al. 2012. Experimental Astronomy 34:385413

Malhan K, Ibata RA. 2018. MNRAS 477:4063-4076

Malhan K, Ibata RA, Martin NF. 2018. MNRAS 481:3442-3455

Marques Oliveira J, Sicardy B, Desmars J, Bertrand T, Lecacheux J, et al. 2019. Triton's temperature profile and time evolution from the October 5th 2017 stellar occultation. In EPSC-DPS Joint Meeting 2019, vol. 2019

Marques Oliveira J, Sicardy B, Meza E, Desmars J, Lecacheux J, et al. 2018. Stellar Occultation of Triton on October 5th, 2017. In European Planetary Science Congress

Massari D, Breddels MA, Helmi A, Posti L, Brown AGA, Tolstoy E. 2018. Nature Astronomy $2: 156-161$

Mateu C, Cooper AP, Font AS, Aguilar L, Frenk C, et al. 2017. MNRAS 469:721-743

Meijer EA, Nijenhuis JN, Vink RJP, Kamphues F, Gielesen W, Coatantiec C. 2009. Picometer metrology for the GAIA Mission. In Astronomical and Space Optical Systems, eds. PG Warren, CJ Marshall, JB Heaney, ET Kvamme, RK Tyson, M Hart, vol. 7439. International Society for Optics and Photonics, SPIE

Meingast S, Alves J. 2019. A\&SA 621:L3

Meingast S, Alves J, Fürnkranz V. 2019. A $\& A$ 622:L13

Meingast S, Alves J, Rottensteiner A. 2020. arXiv e-prints :arXiv:2010.06591

Meza E, Sicardy B, Assafin M, Ortiz JL, Bertrand T, et al. 2019. A 6 A 625:A42

Michalik D, Lindegren L, Hobbs D, Lammers U. 2014. A $\& A$ 571:A85

Mignard F. 2009. The Hundred Thousand Proper Motions Project. GAIA-C3-TN-OCA-FM-040

Mignard F, Klioner S. 2012. A\& A 547:A59

Monet DG. 1988. ARAESA 26:413-440

Mora A, Biermann M, Brown AGA, Busonero D, Carminati L, et al. 2014. Gaia on-board metrology: 
basic angle and best focus. In Space Telescopes and Instrumentation 2014: Optical, Infrared, and Millimeter Wave, eds. JMO Jr., M Clampin, GG Fazio, HA MacEwen, vol. 9143. International Society for Optics and Photonics, SPIE

Mucciarelli A, Monaco L, Bonifacio P, Saviane I. 2017. A\& A 603:L7

Nielsen EL, De Rosa RJ, Wang JJ, Sahlmann J, Kalas P, et al. 2020. AJ 159:71

Noll KS, Brown ME, Weaver HA, Grundy WM, Porter SB, et al. 2020. Detection of a Satellite of the Trojan Asteroid (3548) Eurybates - A Lucy Mission Target. In 51st Lunar and Planetary Science Conference, Lunar and Planetary Science Conference

Oh S, Price-Whelan AM, Hogg DW, Morton TD, Spergel DN. 2017. AJ 153:257

Ostrovski F, Lemon CA, Auger MW, McMahon RG, Fassnacht CD, et al. 2018. MNRAS 473:L116L120

Pedregosa F, Varoquaux G, Gramfort A, Michel V, Thirion B, et al. 2011. Journal of Machine Learning Research 12:2825-2830

Pelisoli I, Vos J. 2019. MNRAS 488:2892-2903

Perek L. 1967. Transactions of the International Astronomical Union 13:60-64

Pérez F, Granger BE. 2007. Computing in Science and Engineering 9:21-29

Perryman M. 2009. Astronomical Applications of Astrometry: Ten Years of Exploitation of the Hipparcos Satellite Data. Cambridge University Press

Perryman M. 2010. The Making of History's Greatest Star Map. Springer

Perryman M. 2011. A\&SA Rev. 19:45

Perryman M. 2012. European Physical Journal H 37:745-792

Perryman M, Hartman J, Bakos GÁ, Lindegren L. 2014. ApJ 797:14

Piskunov AE, Just A, Kharchenko NV, Berczik P, Scholz RD, et al. 2018. A\&\&A 614:A22

Plavin AV, Kovalev YY, Petrov LY. 2019. ApJ 871:143

Poggio E, Drimmel R, Andrae R, Bailer-Jones CAL, Fouesneau M, et al. 2020. Nature Astronomy 4:590-596

Porter SB, Buie MW, Parker AH, Spencer JR, Benecchi S, et al. 2018. AJ 156:20

Price-Whelan AM, Bonaca A. 2018. ApJ 863:L20

Ramos P, Mateu C, Antoja T, Helmi A, Castro-Ginard A, et al. 2020. AESA 638:A104

Ratzenböck S, Meingast S, Alves J, Möller T, Bomze I. 2020. AESA 639:A64

Recio-Blanco A, de Laverny P, Brown AGA, Prusti T, eds. 2018. Astrometry and Astrophysics in the Gaia sky, vol. 330 of IAU Symposium

Reid MJ, Honma M. 2014. ARA \&A 52:339-372

Riello M, De Angeli F, Evans DW, Busso G, Hambly NC, et al. 2018. A ESA 616:A3

Riess AG, Casertano S, Anderson J, MacKenty J, Filippenko AV. 2014. ApJ 785:161

Riess AG, Casertano S, Yuan W, Macri L, Bucciarelli B, et al. 2018. ApJ 861:126

Rioja M, Dodson R. 2019. arXiv e-prints :arXiv:1910.13086

Rioja MJ, Dodson R. 2020. A $\& A$ Rev. 28:6

Risquez D, van Leeuwen F, Brown AGA. 2013. A\&SA 551:A19

Robin AC, Luri X, Reylé C, Isasi Y, Grux E, et al. 2012. A $\& A$ 543:A100

Rodeghiero G, Pott JU, Arcidiacono C, Massari D, Glück M, et al. 2018. MNRAS 479:1974-1985

Rodeghiero G, Sawczuck M, Pott JU, Glück M, Biancalani E, et al. 2019. PASP 131:054503

Röser S, Schilbach E, Goldman B. 2019. A\&3A 621:L2

Rowell N, Davidson M, Lindegren L, van Leeuwen F, Castañeda J, et al. 2020. submitted to A\&A

Ruiz-Lara T, Gallart C, Bernard EJ, Cassisi S. 2020. Nature Astronomy

Sanders JL, Das P. 2018. MNRAS 481:4093-4110

Sartoretti P, Katz D, Cropper M, Panuzzo P, Seabroke GM, et al. 2018. A\&\&A 616:A6

Schönrich R, McMillan P, Eyer L. 2019. MNRAS 487:3568-3580

Schwan H. 2002. A\&\& 387:1123-1134

Sesar B, Fouesneau M, Price-Whelan AM, Bailer-Jones CAL, Gould A, Rix HW. 2017. ApJ 838:107

Sim G, Lee SH, Ann HB, Kim S. 2019. Journal of Korean Astronomical Society 52:145-158 
Simpson JD, De Silva GM, Martell SL, Zucker DB, Ferguson AMN, et al. 2017. MNRAS 471:40874098

Snellen IAG, Brown AGA. 2018. Nature Astronomy 2:883-886

Sohn ST, Anderson J, van der Marel RP. 2012. ApJ 753:7

Spoto F, Tanga P, Bouquillon S, Desmars J, Hestroffer D, et al. 2017. A\&A 607:A21

Spoto F, Tanga P, Carry B. 2019. Direct detections of the Yarkovsky drift with Gaia DR2. In EPSC-DPS Joint Meeting 2019

Stern SA, Weaver HA, Spencer JR, Olkin CB, Gladstone GR, et al. 2019. Science 364:aaw9771

Tang SY, Pang X, Yuan Z, Chen WP, Hong J, et al. 2019. ApJ 877:12

Tanga P, Mignard F. 2012. Planet. Space Sci. 73:5-9

Tanga et al. 2020. in prep.

Taris F, Souchay J, Andrei AH, Bernard M, Salabert M, et al. 2011. A\&\&A 526:A25

Taylor MB. 2005. TOPCAT \& STIL: Starlink Table/VOTable Processing Software. In Astronomical Data Analysis Software and Systems XIV, eds. P Shopbell, M Britton, R Ebert, vol. 347 of Astronomical Society of the Pacific Conference Series

Tepper-García T, Bland-Hawthorn J, Li D. 2020. MNRAS 493:5636-5647

Tian HJ, Gupta P, Sesar B, Rix HW, Martin NF, et al. 2017. ApJS 232:4

Tremaine S. 1999. MNRAS 307:877-883

Tremblay PE, Fontaine G, Fusillo NPG, Dunlap BH, Gänsicke BT, et al. 2019. Nature 565:202-205

Trippe S, Davies R, Eisenhauer F, Schreiber NMF, Fritz TK, Genzel R. 2010. MNRAS 402:11261140

Unwin SC, Shao M, Tanner AM, Allen RJ, Beichman CA, et al. 2008. PASP 120:38

Vallenari A. 2018. Frontiers in Astronomy and Space Sciences 5:11

van Altena WF. 1983. ARAESA 21:131-164

van Altena WF. 2013. Astrometry for Astrophysics. Cambridge University Press

van Altena WF, Lee JT, Hoffleit ED. 1995. The general catalogue of trigonometric stellar parallaxes.

Yale University Observatory

van de Kamp P. 1971. ARA\&SA 9:103

van der Marel RP, Fardal MA, Sohn ST, Patel E, Besla G, et al. 2019. ApJ 872:24

van der Marel RP, Kallivayalil N. 2014. ApJ 781:121

van Horn HM. 1968. ApJ 151:227

Vasilevskis S. 1966. ARA $\& A$ 4:57

Vasiliev E. 2018. MNRAS 481:L100-L104

Vasiliev E. 2019. MNRAS 484:2832-2850

Villa Vélez JA, Brown AGA, Kenworthy MA. 2018. Research Notes of the American Astronomical Society 2:58

Virtanen P, Gommers R, Oliphant TE, Haberland M, Reddy T, et al. 2020. Nature Methods 17:261272

Voggel KT, Seth AC, Sand DJ, Hughes A, Strader J, et al. 2020. ApJ 899:140

WFIRST Astrometry Working Group, Sanderson RE, Bellini A, Casertano S, Lu JR, et al. 2019. Journal of Astronomical Telescopes, Instruments, and Systems 5:044005

Widrow LM, Gardner S, Yanny B, Dodelson S, Chen HY. 2012. ApJ 750:L41

Zacharias N, Finch C, Frouard J. 2017. AJ 153:166

Zari E, Hashemi H, Brown AGA, Jardine K, de Zeeuw PT. 2018. A $\&$ A 620:A172

Zinn JC, Pinsonneault MH, Huber D, Stello D. 2019. ApJ 878:136 